\title{
Steinberg-like characters for finite simple groups
}

\author{
Gunter Malle and Alexandre Zalesski \\ Communicated by Timothy C. Burness
}

\begin{abstract}
Let $G$ be a finite group and, for a prime $p$, let $S$ be a Sylow $p$-subgroup of $G$. A character $\chi$ of $G$ is called $\operatorname{Syl}_{p}$-regular if the restriction of $\chi$ to $S$ is the character of the regular representation of $S$. If, in addition, $\chi$ vanishes at all elements of order divisible by $p, \chi$ is said to be Steinberg-like. For every finite simple group $G$, we determine all primes $p$ for which $G$ admits a Steinberg-like character, except for alternating groups in characteristic 2. Moreover, we determine all primes for which $G$ has a projective $F G$-module of dimension $|S|$, where $F$ is an algebraically closed field of characteristic $p$.
\end{abstract}

\section{Introduction}

Let $G$ be a finite group and, for a prime $p$, let $S$ be a Sylow $p$-subgroup of $G$. A character $\chi$ of $G$ is called Syl $_{p}$-vanishing if $\chi(u)=0$ for every $1 \neq u \in S$; and if, additionally, $\chi(1)=|S|$, then we say that $\chi$ is $\operatorname{Syl}_{p}$-regular. If $\chi(g)=0$ whenever $|g|$ is divisible by $p$, then $\chi$ is called $p$-vanishing; and if, additionally, $\chi(1)=|S|$, then we say that $\chi$ is Steinberg-like. Steinberg-like and $\mathrm{Syl}_{p}$-regular characters for Chevalley groups in defining characteristic $p$ are studied in [17]. Specifically, for all simple groups of Lie type in characteristic $p$ except $B_{n}(q)$, $n=3,4,5$, and $D_{n}(q), n=4,5$, the Steinberg-like characters for the prime $p$ have been determined in [17].

Our main motivation to study this kind of characters is their connection with characters of projective indecomposable modules. The study of projective indecomposable modules of dimension $|S|$ was initiated by Malle and Weigel [13]; they obtained a full classification of such modules for arbitrary finite simple groups $G$ assuming that the character of the module has the trivial character $1_{G}$ as a constituent. In [23], this restriction was removed for simple groups of Lie type with defining characteristic $p$. Some parts of the proofs there were valid not only for characters of projective modules, but also for Steinberg-like or even $\mathrm{Syl}_{p}$-regular characters.

In this paper, we complete the classification of projective indecomposable modules of dimension $|S|$ for simple groups $G$. The first main result is a classification 
of Steinberg-like characters for simple groups, with the sole exception of alternating groups for the prime $p=2$.

Theorem 1.1. Let $G$ be a finite non-abelian simple group, $p$ a prime dividing $|G|$, and let $\chi$ be a Steinberg-like character of $G$ with respect to $p$. Then one of the following holds:

(1) $\chi$ is irreducible, and the triple $(G, p, \chi(1))$ is as in Proposition 3.1;

(2) Sylow p-subgroups of $G$ are cyclic, and $(G, p, \chi(1))$ is as in Proposition 4.4;

(3) $G$ is of Lie type in characteristic $p$ (see [17]);

(4) $p=2$ and $G=\operatorname{PSL}_{2}(q)$ with $q+1=2^{k}$;

(5) $p=2$ and $G=A_{n}, n \geq 8$.

In fact, in many instances, we even classify all $\mathrm{Syl}_{p}$-regular characters. Examples for case (5) when $n=2^{k}$ or $2^{k}+1$ are presented in Corollaries 6.8 and 6.10. We are not aware of any further examples.

The second main result of this paper determines reducible projective modules of simple groups of minimal possible dimension $|G|_{p}$.

Theorem 1.2. Let $G$ be a finite non-abelian simple group, $p$ a prime dividing $|G|$, and $S$ a Sylow p-subgroup of $G$. Then $G$ has a reducible projective $\overline{\mathbb{F}}_{p} G$-module of dimension $|S|$ if and only if one of the following holds:

(1) $G=\operatorname{PSL}_{2}(q), q>4,|S|=q+1$;

(2) $G=\operatorname{PSL}_{n}(q), n$ is an odd prime, $n \nmid(q-1),|S|=\frac{q^{n}-1}{q-1}$;

(3) $G=A_{p},|S|=p \geq 5$;

(4) $G=M_{11},|S|=11$;

(5) $G=M_{23},|S|=23$.

Note that irreducible projective $\overline{\mathbb{F}}_{p} G$-modules of dimension $|G|_{p}$ are in bijection with irreducible characters of defect 0 of that degree, listed in Proposition 3.1 for simple groups.

The paper is built up as follows. After some preliminaries, we recall the classification of irreducible Steinberg-like characters in Section 3 (Proposition 3.1). In Section 4, we classify Syl $p$-regular characters in the case of cyclic Sylow $p$-subgroups (Proposition 4.4); in Section 5, we treat the sporadic groups (Theorem 5.1). The alternating groups are handled in Section 6 (Theorem 6.4 for $p$ odd, and in Section 6.2 some partial results for $p=2$; see Theorems 6.12 and 6.14). The exceptional groups of Lie type are considered in Section 7 (Theorem 7.1). The rest of 
our paper deals with the classical groups of Lie type. We start off in Section 8 by ruling out the remaining possibilities in defining characteristic from [17]. The case of large Sylow $p$-subgroups for non-defining primes $p$ is settled in Section 9. In Section 10, we discuss the small cases when $p>2$, while the proofs of our main theorems are achieved in Section 11 by treating the case when $p=2$.

\section{Preliminaries}

We start off by fixing some notation. Let $\mathbb{F}_{q}$ be the finite field of $q$ elements and $\overline{\mathbb{F}}_{q}$ an algebraic closure of $\mathbb{F}_{q}$. The cardinality of a set $X$ is denoted by $|X|$. The greatest common divisor of integers $m, n$ is denoted by $(m, n)$; if $p$ is a prime, then $|n|_{p}$ is the $p$-part of $n$, that is, $n=|n|_{p} m$, where $(m, p)=1$. If $(m, n)=m$, we write $m \mid n$. We write $|n|_{p}^{k}$ for $\left(|n|_{p}\right)^{k}$.

For a finite group $G, \operatorname{Irr}(G)$ is the set of its irreducible characters, and $\operatorname{Irr}_{1}(G)$ is the set of all linear characters of $G$ (that is, of degree 1 ). We denote by $1_{G}$ the trivial character and by $\rho_{G}^{\text {reg }}$ the regular character of $G$. We write $S \in \operatorname{Syl}_{p}(G)$ to mean that $S$ is a Sylow $p$-subgroup of $G$. A group of order coprime to $p$ is called a $p^{\prime}$-group. Further, $Z(G), G^{\prime}$ denote the centre and the derived subgroup of $G$, respectively.

If $H$ is a subgroup of $G$, then $C_{G}(H), N_{G}(H)$ denote the centraliser and normaliser of $H$ in $G$, respectively. If $\chi$ is a character of $G$, then we write $\left.\chi\right|_{H}$ for the restriction of $\chi$ to $H$. The $H$-level of $\chi$ is the maximal integer $l \geq 0$ such that $\left.\chi\right|_{H}-l \cdot \rho_{H}^{\mathrm{reg}}$ is a proper character of $H$. If a prime $p$ is fixed, then the $p$-level $l_{p}(\chi)$ of $\chi$ is the $S$-level of $\chi$ for $S \in \operatorname{Syl}_{p}(G)$. (For quasi-simple groups with cyclic Sylow $p$-subgroups, irreducible characters of $p$-level $l=1,2$ are studied in $[18,22]$, respectively.) The inner product of characters $\lambda, \mu$ of $G$ is denoted by $(\lambda, \mu)$, sometimes by $(\lambda, \mu)_{G}$. The character of $G$ induced from a character $\mu$ of $H$ is denoted by $\mu^{G}$.

Let $P \leq G$ be finite groups, $N$ a normal subgroup of $P$ and $L=P / N$. Let $F$ be a field and $M$ an $F G$-module. Then $M^{N}:=C_{M}(N)$ becomes an $F L$-module, which is called the generalised restriction of $M$ to $L$ and denoted by $r_{P / N}^{G} M$ in [2, $\S 70 \mathrm{~A}$, p. 667]. If $\beta$ is the Brauer (or ordinary) character of $M$, then we also write $r_{P / N}^{G} \beta$ for the Brauer (or ordinary) character of $L$ afforded by $M^{N}$.

Let $e=e_{p}(q)(p>2,(p, q)=1)$ be the minimal integer $i>0$ such that $q^{i}-1$ is divisible by $p$. If $p=2$ and $q$ is odd, then we set $e_{2}(q)=1$ if $4 \mid(q-1)$, and $e_{2}(q)=2$ if $4 \mid(q+1)$.

The next two lemmas follow from the definitions; here $G$ is a finite group and $S \in \operatorname{Syl}_{p}(G)$. 
Lemma 2.1. Let $\chi$ be a $\mathrm{Syl}_{p}$-regular character of $G$. Then every linear character occurs in $\chi \mid{ }_{S}$ with multiplicity 1 . In particular, $\left(\left.\chi\right|_{S}, 1_{S}\right)=1$. If $S$ is abelian then $\left.\chi\right|_{S}$ is multiplicity free.

Proof. As $\left.\chi\right|_{S}=\rho_{S}^{\text {reg }}$, this follows from the corresponding properties of $\rho_{S}^{\mathrm{reg}}$.

Lemma 2.2. Let $G=G_{1} \times G_{2}$ be a direct product, and let $\chi_{1}$, $\chi_{2}$ be irreducible characters of $G_{1}, G_{2}$. respectively. Then the p-level of $\chi_{1} \otimes \chi_{2}$ is the product of the p-levels of $\chi_{1}$ and $\chi_{2}$.

Lemma 2.3. Let $N$ be a $p^{\prime}$-subgroup of $G$ normalised by $S$. Let $\chi$ be a faithful Steinberg-like character of $G$. Then $N$ is abelian, and $C_{G}(S)=Z(G) Z(S)$.

Proof. Let $H=N S$. Then $\left.\chi\right|_{H}$ is Steinberg-like. Since $H$ is $p$-solvable, every $p$-vanishing character is the character of a projective module [15, Lemma 10.16]. As $\chi(1)=|S|$, the module in question is indecomposable. Then $\left.\chi\right|_{H}$ is induced from an irreducible character $\alpha$, say, of $N$ [15, Theorem 10.13]. As

$$
\alpha^{H}(1)=\alpha(1) \cdot|H: N|=\alpha(1) \cdot|S| \quad \text { and } \quad \chi(1)=|S|,
$$

it follows that $\alpha(1)=1$. Let $N^{\prime}$ be the derived subgroup of $N$. Then $N^{\prime}$ is normal in $H$, and $\alpha\left(N^{\prime}\right)=1$. Therefore, $\left.\alpha^{H}\right|_{N^{\prime}}=|S| \cdot 1_{N^{\prime}}$, that is, $N^{\prime}$ lies in the kernel of $\alpha^{H}$. Since $\chi$ and hence $\left.\chi\right|_{H}=\alpha^{H}$ is faithful, we have $N^{\prime}=1$. So $N$ is abelian as claimed.

Note that $C_{G}(S)=A \times Z(S)$, where $A$ is a $p^{\prime}$-group. Take $N=A$ above, so $H=A \times S$. So now $[N, S]=1$, and $N$ is abelian. It follows that, in any representation afforded by $\alpha^{H}, N$ consists of scalar matrices. As $\chi$ is faithful, we have $[N, G]=1$, as required.

Thus, if $G$ is a simple group, then $C_{G}(S)=Z(S)$ is a necessary condition for $G$ to have a Steinberg-like character.

Remark 2.4. A $p^{\prime}$-subgroup $N$ normalised by a Sylow $p$-subgroup of $G$ is called a $p$-signaliser in the theory of finite groups. Thus Lemma 2.3 tells us that if $G$ admits a faithful Steinberg-like character, then every $p$-signaliser is abelian, and $C_{G}(S)=Z(G) Z(S)$.

Lemma 2.5. Let $G$ be a finite group, $P$ a subgroup with $(|G: P|, p)=1, U$ a normal $p$-subgroup of $P$, and let $L=P / U$. Let $T, S$ be Sylow $p$-subgroups of $L, G$, respectively. Let $\chi$ be a character of $G$ and $\lambda=r_{P / U}^{G}(\chi)$.

(a) If $\left.\chi\right|_{S}=m \cdot \rho_{S}^{\mathrm{reg}}$, then $\left.\lambda\right|_{T}=m \cdot \rho_{T}^{\mathrm{reg}}$. In other words, $l_{p}(\chi)=l_{p}(\lambda)$. In particular, if $\chi$ is $\mathrm{Syl}_{p}$-regular, then so is $\lambda$. 
(b) If $\chi$ is a p-vanishing character of $G$, then $\lambda$ is a p-vanishing character of $L$.

(c) Let $K:=O^{p^{\prime}}(L)$. If $\chi$ is a Steinberg-like or $\mathrm{Syl}_{p}$-regular character of $G$, then so is the character $\left.\lambda\right|_{K}$ of $K$.

Proof. We can assume that $S \leq P$ and $T=S / U$.

(a) As $\left.\chi\right|_{S}=m \cdot \rho_{S}^{\mathrm{reg}}$, it follows that $\left.\lambda\right|_{T}$ coincides with $m \cdot \rho_{T}^{\mathrm{reg}}$, whence the claim.

(b) We have to show that $\lambda$ vanishes at all $p$-singular elements of $L$. Let $M$ be a $\mathbb{C} G$-module afforded by $\chi$. Then $C_{M}(U)=\left\{\frac{1}{|U|} \sum_{u \in U} u x \mid x \in M\right\}$. Observe that if $g \in P$ has projection to $L$ which is not a $p^{\prime}$-element, then $g u$ is not a $p^{\prime}$-element for any $u \in U$. Thus, for any such element $g$, it follows that $\lambda(g)=\frac{1}{|U|} \sum_{u \in U} \chi(g u)=0$ by assumption, whence the claim.

(c) is obvious.

Lemma 2.6. Let $G=G_{1} \times G_{2}$ be a direct product. Suppose that $l_{p}(\sigma) \geq k$ for every non-zero $\mathrm{Syl}_{p}$-vanishing (respectively, $p$-vanishing) character $\sigma$ of $G_{2}$. Then $l_{p}(\chi) \geq k$ for every $\mathrm{Syl}_{p}$-vanishing (respectively, $p$-vanishing) character $\chi$ of $G$.

Proof. Let $S_{1} \in \operatorname{Syl}_{p}\left(G_{1}\right)$. Set $U=S_{1}$ and $P=N_{G}(U)$, so $P=N_{G_{1}}\left(S_{1}\right) \times G_{2}$. Then $L:=P / U=L_{1} \times G_{2}$, where $L_{1}=N_{G_{1}}\left(S_{1}\right) / S_{1}$. Let $\chi$ be a Syl $p^{\text {-vanish- }}$ ing (respectively, $p$-vanishing) character of $G$. Let $\lambda=r_{P / U}^{G}(\chi)$ be the gener-

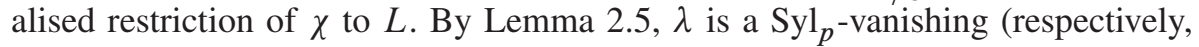
$p$-vanishing) character of $L$, and $l_{p}(\chi)=l_{p}(\lambda)$. Then $l_{p}(\lambda)=l_{p}\left(\left.\lambda\right|_{G_{2}}\right)$ as $L_{1}$ is a $p^{\prime}$-group. By assumption, $l_{p}\left(\left.\lambda\right|_{G_{2}}\right) \geq k$, whence the result.

Lemma 2.7. Let $G=G_{1} \times G_{2}$, where $\left|G_{2}\right|_{p}>1$, and let $\chi$ be a p-vanishing character of $G$. Then $\chi=\sum_{i} \eta_{i} \sigma_{i}$, where $\eta_{i} \in \operatorname{Irr}\left(G_{1}\right)$ are all distinct, and $\sigma_{i}$ are $p$-vanishing characters of $G_{2}$. In addition, $\chi_{1}:=\sum_{i} l_{p}\left(\sigma_{i}\right) \eta_{i}$ is a p-vanishing character of $G_{1}$, and $l_{p}\left(\chi_{1}\right)=l_{p}(\chi)$.

Proof. Write $\chi=\sum_{i} \eta_{i} \sigma_{i}$, where $\eta_{i} \in \operatorname{Irr}\left(G_{1}\right)$ are all distinct, and the $\sigma_{i}$ 's are some characters of $G_{2}$ (reducible, in general). Let $g \in G_{1}$, and let $x \in G_{2}$ be $p$-singular. Then $0=\chi(g x)=\sum_{i} \eta_{i}(g) \sigma_{i}(x)$. As the characters $\eta_{i}$ are linearly independent, it follows that $\sigma_{i}(x)=0$ for every $i$, that is, the $\sigma_{i}$ 's are $p$-vanishing.

In addition, $\left|G_{2}\right|_{p} \sum l_{p}\left(\sigma_{i}\right) \eta_{i}=\sum_{i} \eta_{i} \sigma_{i}(1)=\left.\chi\right|_{G_{1}}$. So $\sum_{i} l_{p}\left(\sigma_{i}\right) \eta_{i}$ is $p$-vanishing. Let $l_{p}(\chi)=m$. Then

$$
\chi(1)=m|G|_{p}=m\left|G_{1}\right|_{p}\left|G_{2}\right|_{p}=\sum \eta_{i}(1) \sigma_{i}(1)=\sum \eta_{i}(1) l_{p}\left(\sigma_{i}\right)|G|_{2}
$$

whence $m\left|G_{1}\right|_{p}=\sum \eta_{i}(1) l_{p}\left(\sigma_{i}\right)$, as required. 
Corollary 2.8. Let $G=G_{1} \times G_{2}$ and $\chi$ be as in Lemma 2.7, and let $S_{i}$ be a Sylow p-subgroup of $G_{i}, i=1,2$. Let $\eta_{1}, \ldots, \eta_{k}$ be the irreducible constituents of $\left.\chi\right|_{G_{1}}$, and $\eta=\eta_{1}+\cdots+\eta_{k}$. Suppose that $l_{p}(\sigma) \geq m$ for every non-zero $p$-vanishing character $\sigma$ of $G_{2}$. Then $l_{p}(\chi) \geq m \cdot \eta(1) /\left|S_{1}\right|$.

Proof. Let $\chi=\sum \eta_{i} \sigma_{i}$ be as in Lemma 2.7. By assumption, $\left.\sigma_{i}\right|_{S_{2}}=m_{i} \cdot \rho_{S_{2}}^{\text {reg }}$, where $m_{i} \geq m$. So $m \cdot \rho_{S_{2}}^{\text {reg }}$ is a subcharacter of $\left.\sigma_{i}\right|_{S_{2}}$. Therefore,

$$
\sum_{i}\left(\left.\eta_{i}\right|_{S_{1}} \cdot m \cdot \rho_{S_{2}}^{\mathrm{reg}}\right)=\left.\left(\sum_{i} \eta_{i}\right)\right|_{S_{1}} \cdot m \cdot \rho_{S_{2}}^{\mathrm{reg}}
$$

is a subcharacter of $\left.\chi\right|_{S_{1} \times S_{2}}$. Now $\chi(1) \geq m \eta(1)\left|S_{2}\right|=m \eta(1)|G|_{p} /\left|S_{1}\right|$. As $\chi(1)$ is a multiple of $|G|_{p}$, we have $\chi(1)=l_{p}(\chi)|G|_{p}$, and the result follows.

Proposition 2.9. Let $G$ be a finite group and $N \triangleleft G$ a normal subgroup such that $G / N$ is a cyclic p-group. Let $\chi$ be a p-vanishing character of $G$. Then

(a) $\chi=\psi^{G}$ for some character $\psi$ of $N$;

(b) if $h \in N$ is p-singular and the conjugacy classes of $h$ in $G$ and in $N$ coincide, then $\psi(h)=0$;

(c) if $\psi$ is G-invariant, then $\psi$ is p-vanishing.

Proof. (a) Let $\lambda \in \operatorname{Irr}(G)$ be a linear character that generates $\operatorname{Irr}(G / N)$. As all elements of $G \backslash N$ are $p$-singular, $\chi$ vanishes on $G \backslash N$. It follows that $\lambda \cdot \chi=\chi$. Thus, if we write $\chi=\sum_{j} a_{j} \chi_{j}$ as a non-negative linear combination of irreducible characters $\chi_{j} \in \operatorname{Irr}(G)$, then $a_{j}$ is constant on orbits under multiplication with $\lambda$. It clearly suffices to show the claim for a single orbit, say $\chi=\sum_{i=1}^{p^{f}} \lambda^{i} \chi^{\prime}$ with $\chi^{\prime} \in \operatorname{Irr}(G)$ and $f$ minimal such that $\lambda^{p^{f}} \chi^{\prime}=\chi^{\prime}$.

Set $M:=\operatorname{ker}\left(\lambda^{p^{f}}\right)$. Then $\left.\chi^{\prime}\right|_{M}$ is irreducible as so is $\chi^{\prime}$, so $\chi=\left(\left.\chi^{\prime}\right|_{M}\right)^{G}$. Now note that $\lambda^{p^{f}}$ generates $\operatorname{Irr}(G / M)$, so $\lambda^{p^{f}}(m) \neq 1$ for $m \notin(M \backslash N)$. Thus, as $\lambda^{p^{f}} \chi^{\prime}=\chi^{\prime}$, it follows that $\chi^{\prime}$ vanishes on $M \backslash N$, and hence $\left.\chi^{\prime}\right|_{M}=\psi^{M}$ is induced from some $\psi \in \operatorname{Irr}(N)$. Then $\chi=\left(\left.\chi^{\prime}\right|_{M}\right)^{G}=\left(\psi^{M}\right)^{G}=\psi^{G}$ as claimed.

(b) For $g \in G$, define the character $\psi^{g}$ of $N$ by $\psi^{g}(x)=\psi\left(g x g^{-1}\right)(x \in N)$. It is well know that $\left.\psi^{G}\right|_{N}$ is a sum of $p^{k}$ characters $\psi^{g}$ for suitable $g \in G$. By assumption, $\psi^{g}(h)=\psi(h)$, and hence $0=\chi(h)=p^{k} \psi(h)$, whence (b).

(c) If $\psi$ is $G$-invariant, then $\psi^{g}=\psi$, and hence $\left.\chi\right|_{N}=p^{k} \cdot \psi$. It follows that $\psi$ is $p$-vanishing, whence the result.

Remark 2.10. Let $G, N, p, \chi, \psi$ be as in Proposition 2.9. Then $\psi$ is not necessarily $p$-vanishing. Indeed, let $C=\langle c\rangle$ be the cyclic group of order 4 , and let $\varepsilon$ be a square root of -1 . Define $\mu_{i} \in \operatorname{Irr}(C)(i=1,2,3,4)$ by $\mu_{i}(c)=\varepsilon^{i}$. 
Then $\sum_{i} \mu_{i}=\rho_{C}^{\mathrm{reg}}$, the regular character of $C$. Let $D$ be the dihedral group of order 8 with normal subgroup $C$. Then $\left(\sum_{i} \mu_{i}\right)^{D}=\rho_{D}^{\text {reg }}$. One observes that $\mu_{1}^{D}=\mu_{3}^{D}$, and hence $\left(2 \mu_{1}+\mu_{2}+\mu_{4}\right)^{D}=\rho_{D}^{\text {reg }}$. However, $2 \mu_{1}+\mu_{2}+\mu_{4}$ is not a 2-vanishing character of $C$.

Corollary 2.11. Let $G, N$ be as in Proposition 2.9, and let $\chi$ be a Steinberg-like character of $G$. Suppose that every irreducible character of $N$ of degree at most $|N|_{p}$ is $G$-invariant. Then $\chi=\psi^{G}$ for some Steinberg-like character $\psi$ of $N$. In particular, if $N$ does not have Steinberg-like characters, then neither has $G$.

Proof. By Proposition 2.9 (a), $\chi=\psi^{G}$ for some character $\psi$ of $N$. Clearly,

$$
\psi(1)=\chi(1) /|G: N|=|G|_{p} /|G: N|=|N|_{p},
$$

so, by assumption, every irreducible constituent of $\psi$ is $G$-invariant. Therefore, so is $\psi$, and the claim follows from Proposition 2.9 (c).

Lemma 2.12. Let $G$ be a finite group and $N \triangleleft G$ a normal subgroup of p-power index. Suppose that $l_{p}(\chi) \geq m$ for some integer $m>0$ and every $p$-vanishing character $\chi$ of $G$. Then $l_{p}\left(\chi_{1}\right) \geq m$ for every $p$-vanishing character $\chi_{1}$ of $N$.

Proof. Suppose the contrary. Let $\chi_{1}$ be a $p$-vanishing character of $N$ such that $l_{p}\left(\chi_{1}\right)<m$. Then the induced character $\chi_{1}^{G}$ is $p$-vanishing, and

$$
l_{p}\left(\chi_{1}^{G}\right)=l_{p}\left(\chi_{1}\right)<m .
$$

This is a contradiction.

The following fact is well known.

Lemma 2.13. Let $G$ be a finite group and $N \triangleleft G$ a normal subgroup of p-power index. Let $F$ be an algebraically closed field of characteristic p. Let $\Phi$ be a projective indecomposable $F G$-module. Then $\Phi=\Psi^{G}$, where $\Psi$ is a projective indecomposable $F N$-module and $l_{p}(\Psi)=l_{p}(\Phi)$.

Proof. It is well known that induction sends projective modules to projective modules. Furthermore, by Green's indecomposability theorem [4, Theorem 3.8], induction from normal subgroups of $p$-power index preserves indecomposability. So, if $\Psi$ is an indecomposable direct summand of $\left.\Phi\right|_{N}$, then $\Psi$ is projective, $\Psi^{G}$ is projective indecomposable, and so $\Psi^{G}=\Phi$. The statement $l_{p}(\Psi)=l_{p}(\Phi)$ also follows as $|G: N|=|G: N|_{p}$ by assumption. 


\section{Irreducible Steinberg-like characters for simple groups}

In this section, we complete the list of irreducible characters of simple groups $G$ of degree $|G|_{p}$. For this, it suffices to extract the characters of degree $|G|_{p}$ from the list of irreducible characters of prime-power degree obtained in [14, Theorem 1.1]. This list already appeared in [24, Proposition 2.8], where the case with $p=3$, $G={ }^{2} F_{4}(2)^{\prime}$ was inadvertently omitted.

Note that an irreducible character is Steinberg-like if and only if it is $\mathrm{Syl}_{p^{-}}$ regular.

Proposition 3.1. Let $G$ be a non-abelian simple group. Suppose that $G$ has an irreducible $\mathrm{Syl}_{p}$-regular character $\chi$. Then one of the following holds:

(1) $G$ is a simple group of Lie type in characteristic $p$, and $\chi$ is its Steinberg character;

(2) $G=\mathrm{PSL}_{2}(q), q$ even, and $p=\chi(1)=q \pm 1$, or $G=\mathrm{SL}_{2}(8), p=3$ and $\chi(1)=9$;

(3) $G=\operatorname{PSL}_{2}(q), q$ odd, $\chi(1)=\frac{q \pm 1}{2}$ is a p-power for $p>2$, or $p=2$ and $\chi(1)=q \pm 1$ is a 2-power;

(4) $G=\operatorname{PSL}_{n}(q), q>2, n$ is an odd prime, $(n, q-1)=1$, such that $\chi(1)=\frac{q^{n}-1}{q-1}$ is a p-power;

(5) $G=\operatorname{PSU}_{n}(q), n$ is an odd prime, $(n, q+1)=1$, such that $\chi(1)=\frac{q^{n}+1}{q+1}$ is a p-power;

(6) $G=\operatorname{PSp}_{2 n}(q), n>1, q=r^{k}$ with $r$ an odd prime, and $k n$ is a 2-power such that $\chi(1)=\frac{q^{n}+1}{2}$ is a p-power;

(7) $G=\operatorname{PSp}_{2 n}$ (3), $n>2$ is a prime such that $\chi(1)=\frac{3^{n}-1}{2}$ is a p-power;

(8) $G=A_{p+1}$ and $\chi(1)=p$;

(9) $G=\operatorname{Sp}_{6}(2)$ and $\chi(1)=7$;

(10) $G \in\left\{M_{11}, M_{12}\right\}$ and $\chi(1)=11$;

(11) $G \in\left\{M_{11}, \operatorname{PSL}_{3}(3)\right\}$ and $\chi(1)=16$;

(12) $G \in\left\{M_{24}, \mathrm{Co}_{2}, \mathrm{Co}_{3}\right\}$ and $\chi(1)=23$;

(13) $G={ }^{2} F_{4}(2)^{\prime}$ and $\chi(1)=27$;

(14) $G=\mathrm{PSU}_{3}(3) \cong G_{2}(2)^{\prime}$ and $\chi(1)=32$;

(15) $G=G_{2}(3)$ and $\chi(1)=64$. 
The problem of determining the minimal degree of irreducible characters of $p$ defect 0 looks much more complicated.

Remark 3.2. Let us point out the following cases not explicitly mentioned in Proposition 3.1:

$$
\mathrm{SL}_{3}(2) \cong \mathrm{PSL}_{2}(7), \quad A_{6} \cong \mathrm{PSL}_{2}(9), \quad \mathrm{PSU}_{4}(2) \cong \mathrm{PSp}_{4}(3), \quad A_{8} \cong \mathrm{SL}_{4}(2)
$$

\section{Cyclic Sylow $p$-subgroups}

In this section, we determine the reducible Steinberg-like characters for simple groups with cyclic Sylow $p$-subgroups.

Proposition 4.1. Let $G$ be a finite group with a cyclic TI Sylow p-subgroup $S$, and assume that $N_{G}(S) / S$ is abelian. Then $l_{p}(\tau)=\lfloor\tau(1) /|S|\rfloor$ for all $\tau \in \operatorname{Irr}(G)$.

Proof. Let $N:=N_{G}(S)$. By assumption, $N / S$ is abelian of order prime to $p$, so it has $|N: S|$ irreducible $p$-Brauer characters of degree 1 . Hence, each of the corresponding PIMs of $N$ has dimension $|S|$. Since the Brauer tree for any $p$-block of $N$ is a star, all PIMs are uniserial [4, Chapter VII, Corollary 2.22]. But then, by [4, Chapter I, Theorem 16.14], any indecomposable $F N$-module, where $F$ is a sufficiently large field of characteristic $p$, is a quotient of a PIM, so has dimension strictly smaller than $|S|$ if it is not projective.

Now let $\tau \in \operatorname{Irr}(G)$. If $\tau$ is of $p$-defect zero, $\left.\tau\right|_{S}$ is a multiple of $\rho_{S}^{\text {reg }}$, and the claim follows. Else, $\tau$ lies in a block of full defect, and there exists an indecomposable $F G$-module $X$ with lift $\tau$ [4, Chapter I, Theorem 17.12]. Then $\left.X\right|_{F N}=Y \oplus P$, where $P$ is projective (and hence of dimension divisible by $|S|$ ) and $Y$ is the Green correspondent of $X$, an indecomposable, non-projective $F N$-module [4, Chapter VII, Lemma 1.5]. Thus, $\operatorname{dim} Y<|S|$ by what we said before, so $\tau(1) /|S| \leq l_{p}(\tau)<\tau(1) /|S|+1$, and the claim follows.

Lemma 4.2. Let $G$ be a non-abelian simple group. Let $p$ be a prime such that a Sylow p-subgroup of $G$ is cyclic. Let $\mu$ denote the minimal degree of any nonlinear irreducible character of $G$. Then $2 \mu>|G|_{p}$, except in the case where $G=\operatorname{PSL}_{2}(p), p \equiv 3(\bmod 4)$ and $\mu=\frac{p-1}{2}$.

Proof. The values of $\mu=\mu(G)$ for every simple group $G$ are either known explicitly or there is a good lower bound. For the sporadic simple groups one can inspect [1], for the alternating groups $A_{n}$ we have $\mu\left(A_{n}\right)=n-1$ for $n>5$, and $\mu\left(A_{5}\right)=3$, for simple groups $G$ of Lie type the values $\mu(G)$ are listed in [20]. The lemma follows by comparison of these data with $|G|_{p}$. 
Proposition 4.3. Let $p$ be a prime, and let $G$ be a non-abelian simple group with a cyclic Sylow p-subgroup $S$. Let $\chi$ be a $\mathrm{Syl}_{p}$-regular character of $G$. Then one of the following holds:

(1) $\chi$ is irreducible of degree $|G|_{p}$;

(2) $\left(\chi, 1_{G}\right)=1, \tau:=\chi-1_{G}$ is irreducible and $\left(\left.\tau\right|_{S}, 1_{S}\right)=0$; or

(3) $G=\operatorname{PSL}_{2}(p), p \equiv 3(\bmod 4)$ and $\chi=1_{G}+\tau_{1}+\tau_{2}$, where $\tau_{1}, \tau_{2}$ are distinct irreducible characters of degree $\frac{p-1}{2}$.

Proof. Suppose that $\chi$ is reducible. The result for $G=\operatorname{PSL}_{2}(p)$ easily follows by computation with the character table of this group. Suppose $G \not \operatorname{PSL}_{2}(p)$. Let $\tau \neq 1_{G}$ be an irreducible constituent of $\chi$. By Lemma $4.2, \chi=\tau+k \cdot 1_{G}$, where $k=|G|_{p}-\tau(1)$. Therefore, $1_{G}$ is a constituent of $\chi$. By Lemma 2.1, $k=1$ and $\left(\left.\tau\right|_{S}, 1_{S}\right)=0$.

Proposition 4.4. Let $p$ be a prime, and let $G$ be a non-abelian simple group with a cyclic Sylow p-subgroup $S$. Then $G$ has a reducible $S_{1}{ }_{p}$-regular character $\chi$ if and only if one of the following holds:

(1) $G=\operatorname{PSL}_{2}(q), q>4$ even, $|S|=q+1$;

(2) $G=\operatorname{PSL}_{2}(p),|S|=p>5$;

(3) $G=\operatorname{PSL}_{n}(q), n$ is an odd prime, $n \nmid(q-1),|S|=\frac{q^{n}-1}{q-1}$;

(4) $G=\operatorname{PSU}_{n}(q), n$ is an odd prime, $n \nmid(q+1),|S|=\frac{q^{n}+1}{q+1}$;

(5) $G=A_{p},|S|=p \geq 5$;

(6) $G=M_{11},|S|=11$; or

(7) $G=M_{23},|S|=23$.

Furthermore, in each case (1)-(7), $C_{G}(S)=S$, and $\chi$ is Steinberg-like. In addition, $\chi-1_{G}$ is an irreducible character of $G$ unless, possibly, when (2) holds, when $\chi-1_{G}$ may be the sum of two irreducible constituents of equal degree.

Proof. The additional statement follows from Proposition 4.3. If $\chi-1_{G}$ is reducible, we have case (3) of Proposition 4.3. So we may assume that $\tau=\chi-1_{G}$ is irreducible and thus that $\left(\left.\tau\right|_{S}, 1_{S}\right)=0$. The irreducible characters of $G$ of level 0 are determined in [22, Theorem 1.1], so $\tau$ belongs to the list in [22, Theorem 1.1]. If we drop from that list the characters of degree other than $|S|-1$, the remaining cases are given in the statement of the proposition. (Note that the list in [22, Theorem 1.1] includes quasi-simple groups, so one first needs to delete the representations that are non-trivial on the centre. For instance, if $G=\operatorname{PSp}_{2 n}(q)$, then 
$|S|=\frac{q^{n}+1}{2}$ is odd, and hence $\tau(1)=\chi(1)-1=|S|-1$ is even. However, every irreducible representation of $\operatorname{Sp}_{2 n}(q)$ of even degree $\frac{q^{n}-1}{2}$ is faithful. In other words, $G$ has no irreducible representation of even degree $\frac{q^{n}-1}{2}$. In contrast, there do exist irreducible representations of $G=\operatorname{PSL}_{n}(q)$ and $\operatorname{PSU}_{n}(q)$ for $n$ odd of degree $|S|-1$.)

To prove the converse, we have to show that, in each case, $1_{G}+\tau$ is $\operatorname{Syl}_{p^{-}}$ regular, that is, $\left.\chi\right|_{S}=\rho_{S}^{\text {reg }}$. Let $\Psi$ be a representation of $G$ afforded by $\tau$. Let $s \in S$ with $S=\langle s\rangle$. By [22, Corollary 1.3(2)], the multiplicity of every eigenvalue of $\Psi(s)$ is 1 . As det $\Psi(s)=1$, it follows that 1 is not an eigenvalue of $\Psi(s)$. Therefore, $\left.\chi\right|_{S}=\rho_{S}^{\text {reg }}$, as required.

Next, we show that $C_{G}(S)=S$. In cases (6) and (7), this follows by inspection in [1]. The cases (1), (2) and (5) are trivial. In cases (3) and (4), one can take the preimage $T$, say, of $S$ in $G_{1}=\mathrm{SL}_{n}(q), \mathrm{SU}_{n}(q)$, respectively. Then $T$ is irreducible on the natural module for $G_{1}$. The groups $C_{G_{1}}(T)$ are described by Huppert [8, Sätze 4, 5]. It easily follows that $T$ is self-centralising in $G_{1}$. Then $C_{G}(S)=S$ unless $[g, T] \subseteq Z\left(G_{1}\right)$ for some $g \in N_{G_{1}}(T) \backslash T$. By order consideration, $S$ is a Sylow $p$-subgroup of $G$, so $g$ is not a $p$-element. Let $t \in T$. Then $\left[g, t^{i}\right]=\left[g^{i}, t\right]=1$ for $i=|S|$, so $g|S| \in C_{G_{1}}(T)=T$ by the above. This is a contradiction as $S$ is a Sylow $p$-subgroup.

It follows that every element of $G$ is either a $p$ - or a $p^{\prime}$-element. Therefore, $\chi$ is Steinberg-like if and only if $\left.\chi\right|_{S}=\rho_{S}^{\mathrm{reg}}$.

Lemma 4.5. Under the assumptions and in the notation of Proposition 4.4, we have the following:

(a) $\chi$ is unique unless (2) or (6) holds;

(b) $\chi$ is the character of a projective module when (1), (3), (5) or (7) holds;

(c) $\chi-1_{G}$ is a proper character, and if $m$ is the minimal degree of a non-linear character of $G$, then either $m=\chi(1)-1$, or (1) holds and $m=\chi(1)-2$, or (2) holds and $m=\frac{\chi(1) \pm 1}{2}$.

Proof. (a) Let $\tau=\chi-1_{G}$. Then $\tau(1)=|S|-1$ and $\tau$ is irreducible unless (2) holds. We show that an irreducible character of this degree is unique unless (2) or (6) holds. If $G=M_{23}$, this follows from the character table of this group; for $A_{p}$, this is well known. For $G=\operatorname{PSL}_{n}(q), n>2$, and $\operatorname{PSU}_{n}(q), n>2$, this is observed in [20, Table II].

In case (2), the number of characters equals the number of irreducible characters of degree $p-1$, which is $\frac{p-3}{4}$ if $p \equiv 3(\bmod 4)$; otherwise, $\frac{p-1}{4}$. If $G=M_{11}$, then there are three Steinberg-like characters; see [1]. 
(b) Recall that the principal projective indecomposable module is the only PIM whose character contains $1_{G}$ as a constituent. All the characters $\chi$ in Proposition 4.4 contain $1_{G}$ as a constituent. Therefore, if $\chi$ is the character of a projective module $\Phi$, say, then $\Phi$ is indecomposable and principal. So we compare the list of characters $\chi$ in Proposition 4.4 with the main result of [13]. The comparison rules out case (4) of Proposition 4.4. Furthermore, if $G$ admits at least two Steinberg-like characters, then at most one of them can be the character of a projective module. By (a), this leaves us with cases (1), (5) and (7). As, in each of these cases, $\chi$ is unique, it must be the character of the principal projective indecomposable module listed in [13].

(c) This follows by inspection in [20, Table II].

Remark 4.6. The group $G=\operatorname{PSL}_{2}(p)$ has several $\operatorname{Syl}_{p}$-regular characters; all of them are Steinberg-like, and only one of them is projective.

\section{Sporadic groups}

Theorem 5.1. Let $G$ be a sporadic simple group. Then $G$ does not have a reducible $\mathrm{Syl}_{p}$-regular character unless one of the following holds:

(1) $G=M_{12}, p=3$, four characters with constituents of degrees 11 and 16 each, all Steinberg-like;

(2) $G=M_{24}, p=2$, six characters, none of them Steinberg-like;

(3) $G=M_{11},|S|=11$;

(4) $G=M_{23},|S|=23$.

Proof. For most groups and primes, by [1], there is a conjugacy class of non-trivial $p$-elements taking strictly positive value at all irreducible characters of degree at most $|G|_{p}$. In a few cases, like in $\mathrm{Co}_{3}$ and $\mathrm{Fi}_{23}$ at $p=3$, or $\mathrm{Co}_{1}$ and $J_{4}$ at $p=2$, one has to solve a little linear system of equations for non-negative integral solutions. The only cases where such solutions exist are listed in the statement. Note that cases (3) and (4) occur also in Proposition 4.4.

\section{Alternating groups}

In this section, we consider Steinberg-like characters of alternating groups.

\subsection{Alternating groups for $p>2$}

For odd primes, we give a short proof using a recent result of Giannelli and Law [5] which replaces our earlier more direct proof. 
Lemma 6.1. Let $G=A_{p}, p>3$, and $\chi \in \operatorname{Irr}(G)$. Then $l_{p}(\chi)=\lfloor\chi(1) / p\rfloor$. In addition, $l_{p}(\chi) \neq 1$ for $p>7$ (this fact has also been observed in [18]).

Proof. The first part is just Proposition 4.1. In addition, if $p>7$, then $G$ has no irreducible character of degree $d$ for $p \leq d<2 p$. This implies the claim.

Lemma 6.2. Let $n=k p$, where $p>5$ and $k<p$. Let $G=A_{n}$, and let $\chi$ be a $p$-vanishing character. Then $l_{p}(\chi) \geq 2^{k-1}$, equivalently, $\chi(1) \geq 2^{k-1}|G|_{p}$.

Proof. For $k=1$, the lemma is trivial. Let $k>1$. Let $X_{1} \cong A_{p}, X_{2} \cong A_{n-p}$ be commuting subgroups of $G$. Set $X=X_{1} X_{2}$. Then $\left.\chi\right|_{X}=\sum \eta_{i} \sigma_{i}$, where the $\sigma_{i}$ 's are $p$-vanishing characters of $X_{2}$ and the $\eta_{i}$ 's are distinct irreducible characters of $X_{1}$ (Lemma 2.7). By induction, $l_{p}\left(\sigma_{i}\right) \geq 2^{k-2}$. If $l_{p}\left(\eta_{i}\right) \geq 2$ for some $i$, then $l_{p}(\chi) \geq l_{p}\left(\eta_{i} \sigma_{i}\right) \geq 2^{k-1}$. By Lemma 6.1, if $l_{p}\left(\eta_{i}\right)<2, p>7$, then $l_{p}\left(\eta_{i}\right)=0$, and hence either $\eta_{i}=1_{X_{1}}$ or $\eta_{i}$ is the unique irreducible character of degree $p-1$. (If $p=7$, then we may have $\eta_{i}(1)=10$; see [18].)

Suppose the lemma is false and $p>7$. Then we can rearrange the above to get

$$
\left.\chi\right|_{X}=1_{X_{1}} \cdot \sigma_{1}+\eta_{2} \cdot \sigma_{2},
$$

where $\eta_{2}(1)=p-1$ and $\sigma_{1}, \sigma_{2}$ are $p$-vanishing characters of $X_{2}$. It follows that $\left.\chi\right|_{X_{1}}$, as well as $\left.\tau\right|_{X_{1}}$ for every irreducible constituent $\tau$ of $\chi$, contains no irreducible constituent distinct from $1_{X_{1}}, \eta_{2}$. It is well known and easily follows from the branching rule that this implies $\tau(1)=n-1$ or 1 . Recall that $G$ has a single character of degree $n-1$. Therefore, $\chi=a \cdot 1_{G}+b \cdot \tau$, where $\tau(1)=n-1$. Let $x \in X_{1}$ be of order $p$. Then $\tau(x)=n-p-1>0$, which implies $\chi(x)>0$.

Suppose $p=7$. Then $\eta_{i}(1) \in\{1,6,10\}$. There are two irreducible characters of $X_{1}$ of degree 10; let us denote them by $\eta_{3}, \eta_{3}^{\prime}$. Therefore, assuming the lemma is false, we can write $\left.\chi\right|_{X}=1_{X} \cdot \sigma_{1}+\eta_{2} \sigma_{2}+\eta_{3} \sigma_{3}+\eta_{3}^{\prime} \sigma_{4}$. Let $1 \neq x \in X_{1}$ be a $p$-element. Then $\eta_{3}(x)=\varepsilon+\varepsilon^{4}+\varepsilon^{2}$ and $\eta_{3}^{\prime}(x)=\varepsilon^{-1}+\varepsilon^{-4}+\varepsilon^{-2}$, where $\varepsilon$ is some primitive 7 th root of unity. Since $\chi(x)$ and $\eta_{2}(x)$ are integers, so is $\eta_{3}(x) \sigma_{3}(1)+\eta_{3}^{\prime}(x) \sigma_{4}(1)$. This implies $\sigma_{3}(1)=\sigma_{4}(1)$. Then

$$
\chi(1)=\sigma_{1}(1)+(p-1) \sigma_{2}(1)+20 \sigma_{3}(1)>14 \sigma_{3}(1),
$$

and the lemma follows unless $\sigma_{3}(1)=0$. If $\sigma_{3}(1)=0$, then

$$
\left.\chi\right|_{X}=1_{X_{1}} \cdot \sigma_{1}+\eta_{2} \cdot \sigma_{2},
$$

and the above argument applies.

Lemma 6.3. Let $p \geq 3$ be odd, and let $\lambda$ be a hook partition of $n \geq 2 p$. Then the corresponding character $\chi^{\lambda}$ of $S_{n}$ takes a positive value on $p$-cycles. 
Proof. It is well known that any hook character $\chi^{\lambda}$ is the $m$ th exterior power, for some $0 \leq m \leq n-1$, of the irreducible reflection character $\rho_{n}$ of $S_{n}$ (the constituent of degree $n-1$ of the natural permutation character $\pi_{n}$ ). Let $Y=Y_{1} \times Y_{2}$, with $Y_{1}=S_{p}$ and $Y_{2}=S_{n-p}$, be a Young subgroup of $S_{n}$ and $g=g^{\prime} \times 1 \in Y$ a $p$-cycle. Clearly, $\left.\rho_{n}\right|_{Y}=\rho_{p} \otimes 1_{Y_{2}}+1_{Y_{1}} \otimes\left(\pi_{n-p}\right)$, and $\Lambda^{i}\left(\rho_{p}\right)\left(g^{\prime}\right)=(-1)^{i}$ for $i<p, \Lambda^{i}\left(\rho_{p}\right)\left(g^{\prime}\right)=0$ for $i \geq p$. Thus,

$$
\begin{aligned}
\chi^{\lambda}(g)=\Lambda^{m}\left(\rho_{n}\right)(g) & =\sum_{i=0}^{m} \Lambda^{i}\left(\rho_{p}\right)\left(g^{\prime}\right) \Lambda^{m-i}\left(\pi_{n-p}\right)(1) \\
& =\sum_{i=0}^{\min (p-1, m)}(-1)^{i}\left(\begin{array}{c}
n-p \\
m-i
\end{array}\right),
\end{aligned}
$$

which clearly is positive for $m \leq \frac{n-p}{2}$ since the binomial coefficients are (strictly) increasing up to the middle. Now observe that it suffices to prove the claim for $n=2 p$ since the restriction of a hook character from $S_{n}$ to $S_{n-1}$ only contains hook characters. But, for $n=2 p$, we are done since, by symmetry, we may assume that $m \leq p=\frac{n-p}{2}$.

Theorem 6.4. Let $p$ be odd and $G=A_{n}$ with $n>\max \{6, p+1\}$. Then $G$ has no $\mathrm{Syl}_{p}$-regular character. If $n=p+1>4$, then every $\mathrm{Syl}_{p}$-regular character of $G$ is irreducible unless $(n, p)=(6,3)$.

Proof. If $p \leq n<2 p$, the Sylow $p$-subgroups of $G$ are cyclic, and so the claim is in Proposition 4.4. Now assume that $n \geq 2 p$, and let $S$ be a Sylow $p$-subgroup of $S_{n}$. First assume that $n \neq p^{k}$ for some $k \geq 2$ and that $n>10$ when $p=3$. Then, by the main result of [5], the restriction of any irreducible character of $S_{n}$ to $S$ contains the trivial character, and the same is clear to be true for the restriction of any irreducible character of $A_{n}$ to $S$. So, by Lemma 2.1, any $S_{1}{ }_{p}$-regular character of $A_{n}$ is irreducible.

Now assume that $n=p^{k}$ for some $k \geq 2$, and $n>10$ when $p=3$. Then, again by [5, Theorem A], the only irreducible characters of $S_{n}$ whose restriction to $S$ does not contain the trivial character are the two characters of degree $n-1$. So the only irreducible character of $A_{n}$ whose restriction to $S$ does not contain the trivial character is $\psi$ of degree $n-1$. Hence a Syl $p$-regular character $\chi$ of $A_{n}$ has the form $\chi=a \psi+\psi^{\prime}$, for some $a \geq 0$ and some $\psi^{\prime} \in \operatorname{Irr}\left(A_{n}\right)$. Let $g \in A_{n}$ be a $p^{k}$-cycle. Then $\psi(g)=-1$, and by the Murnaghan-Nakayama rule, any irreducible character of $S_{n}$ takes value 0 or \pm 1 on $g$. In particular, if $\chi$ is reducible, then we have that $a=1$ and $\psi^{\prime}(g) \neq 0$. But then $\psi^{\prime}$ is parameterised by a hook partition, of degree $\left(\begin{array}{c}n-1 \\ m\end{array}\right)$ for some $m \leq n$. But then $\chi$ takes positive values on $p$-cycles by Lemma 6.3, a contradiction. 
Finally, the cases when $p=3$ and $6 \leq n \leq 10$ can easily be checked individually. For example, all irreducible characters of $A_{9}$ of degree at most 81 are nonnegative on class $3 \mathrm{C}$, and those which vanish there are positive either on class $3 \mathrm{~B}$ or $3 \mathrm{~A}$. So $A_{9}$ has no $\mathrm{Syl}_{3}$-regular character. As $A_{10}$ has the same Sylow 3-subgroup, this also deals with $n=10$.

Corollary 6.5. Let $G$ be a finite group and $p>2$. Suppose that $G$ has a subgroup $P$ containing a Sylow p-subgroup of $G$ and such that $P / O_{p}(P) \cong A_{n}$ with $n>\max \{6, p+1\}$. Then $G$ has no Steinberg-like character.

Proof. This follows from Lemma 2.5 and Theorem 6.4.

\subsection{Alternating groups for $p=2$}

The situation is more complicated in the case of $p=2$, and we do not have complete results. This is in part due to the existence of an infinite family of examples which we now construct.

Set $\Gamma=\sum_{i=1}^{n} \Gamma_{i}$, where $\Gamma_{i}$ is the irreducible character of $S_{n}$ corresponding to the partition $\left[i, 1^{n-i}\right]$ for $i>1$, and $\left[1^{n}\right]$ for $i=1$. So the Young diagram $\gamma_{i}$ of $\Gamma_{i}$ is a hook with leg length $n-i$, and

$$
\Gamma_{i}(1)=\frac{n !}{n(n-i) !(i-1) !}=\left(\begin{array}{c}
n-1 \\
i-1
\end{array}\right),
$$

so $\Gamma(1)=\sum_{i=1}^{n} \Gamma_{i}(1)=2^{n-1}$.

Lemma 6.6. Let $0<m<n$, where $m$ is even, and $g=c h \in S_{m} \times S_{n-m} \leq S_{n}$, where $c$ is an $m$-cycle and $h$ fixes all letters moved by c. Let $\Gamma_{k}^{n-m} \in \operatorname{Irr}\left(S_{n-m}\right)$ correspond to the hook partition $\left[k, 1^{n-m-k}\right]$. Then

$$
\Gamma_{i}(g)= \begin{cases}-\Gamma_{i}^{n-m}(h) & \text { if } i \leq m, \\ \Gamma_{i-m}^{n-m}(h) & \text { if } n-m<i, \\ \Gamma_{i-m}^{n-m}(h)-\Gamma_{i}^{n-m}(h) & \text { if } m<i \leq n-m .\end{cases}
$$

Proof. One observes that the restriction of $\Gamma_{i}$ to $S_{m} \times S_{n-m}$ is a sum of irreducible characters $\sigma \tau$, where $\sigma, \tau$ are irreducible characters of $S_{m}, S_{n-m}$, respectively, and both $\sigma, \tau$ are hook characters of the respective groups (see [9, Lemma 21.3]). Next, we use [9, Lemma 21.1] which states that

$$
\Gamma_{i}(g)=\sum_{j}(-1)^{j} \chi_{v_{j}}(h),
$$


where $\chi_{v_{j}} \in \operatorname{Irr}\left(S_{n-m}\right), v_{j}$ is the Young diagram of $\chi_{v_{j}}$, and $v_{j}$ is such that $\gamma_{i} \backslash v_{j}$ is a skew $m$-hook with leg length $j$. In our case, $\gamma_{i}$ is a hook, so the rim of $\gamma_{i}$ is $\gamma_{i}$ itself. By definition, a skew hook is connected, so it is either a row or a column in our case, and hence $j=0$ or $j=m-1$. (A column hook of length $m$ has leg length $m-1$, which is odd as $m$ is even.) If $j=0, m-1$, then $v_{j}=\left[i-m, 1^{n-i}\right],\left[i, 1^{n-i-m}\right]$, respectively. This is a proper diagram if and only if $i>m$, respectively, $n-i \geq m$. So, if $n-i<m$, then

$$
v_{j}=\left[i-m, 1^{n-i}\right], \quad j=0,
$$

and $\Gamma_{i}(g)=\chi_{v_{j}}(h)=\Gamma_{i-m}^{n-m}(h)$; if $i \leq m$, then

$$
v_{j}=\left[i, 1^{n-i-m}\right], \quad j=m-1,
$$

and $\Gamma_{i}(g)=-\chi_{v_{j}}(h)=-\Gamma_{i}^{n-m}(h)$; if $m<i \leq n-m$, then

$$
v_{j} \in\left\{\left[i-m, 1^{n-i}\right],\left[i, 1^{n-i-m}\right]\right\}
$$

and $\Gamma_{i}(g)=\Gamma_{i-m}^{n-m}(h)-\Gamma_{i}^{n-m}(h)$, as claimed.

Proposition 6.7. Suppose that $n$ is even. Then

(a) $\Gamma$ is a 2-vanishing character of $S_{n}$;

(b) $\Gamma$ is Steinberg-like if and only if $n=2^{k}$ for some integer $k>0$.

Proof. (a) Let $g \in S_{n}$ be of even order. Suppose first that $g$ is a cycle of length $n$. By [9, Lemma 21.1], $\Gamma_{i}(g)=(-1)^{n-i}$, so $\Gamma(g)=0$.

Suppose that $g$ is not a cycle of length $n$. Then we can express $g$ as the product $c h$ of a cycle $c$ of even size $m$, say, and an element $h$ fixing all letters moved by $c$. Then $g \in S_{m} \times S_{n-m}$. By Lemma 6.6, we have

$$
\begin{aligned}
\Gamma(g)=\sum_{i=1}^{n} \Gamma_{i}(g) & =\sum_{i=m+1}^{n} \Gamma_{i-m}^{n-m}(h)-\sum_{i=1}^{n-m} \Gamma_{i}^{n-m}(h) \\
& =\sum_{k=1}^{n-m} \Gamma_{k}^{n-m}(h)-\sum_{i=1}^{n-m} \Gamma_{i}^{n-m}(h)=0 .
\end{aligned}
$$

(b) If $n=2^{k}$, then $\left|S_{n}\right|_{2}=2 \cdot\left|S_{n / 2}\right|_{2}^{2}$. As $\left|S_{2}\right|_{2}=2$, by induction, we have

$$
\left|S_{n}\right|_{2}=2 \cdot\left(2^{2^{k-1}-1}\right)^{2}=2^{2^{k}-1}=2^{n-1} .
$$

Write $n=2^{k}+l$, where $0<l<2^{k}$. Then $\left|S_{n}\right|_{2}=\left|S_{2^{k}}\right|_{2} \cdot\left|S_{l}\right|_{2}$. By induction, $\left|S_{l}\right|_{2} \leq 2^{l-1}$, so

$$
\left|S_{n}\right|_{2}=2^{2^{k}-1} \cdot\left|S_{l}\right|_{2} \leq 2^{\left(2^{k}-1\right)+(l-1)}=2^{2^{k}+l-2}=2^{n-2} .
$$

The statement follows as $\Gamma(1)=2^{n-1}$. 
Corollary 6.8. Let $n$ be even and $\Gamma^{0}=\left.\sum_{i=1}^{n / 2} \Gamma_{i}\right|_{A_{n}}$. Then $\Gamma^{0}$ is a 2-vanishing character of $A_{n}$. If $n=2^{k}$, then this character is Steinberg-like.

Proof. The characters $\Gamma_{i}$ remain irreducible under restriction to $A_{n}$ and

$$
\left.\Gamma_{i}\right|_{A_{n}}=\left.\Gamma_{n-i+1}\right|_{A_{n}} .
$$

It follows that $\left.\Gamma\right|_{A_{n}}=2 \Gamma^{0}$. Therefore, $\Gamma^{0}(g)=\Gamma(g) / 2=0$ by Proposition 6.7 for elements $g$ of even order. The last claim follows from Proposition 6.7 (b).

Suppose that $n$ is odd. Set

$$
\begin{aligned}
\Gamma^{e} & =\sum_{i=1}^{(n-1) / 2} \Gamma_{2 i}=\Gamma_{2}+\Gamma_{4}+\cdots+\Gamma_{n-1}, \\
\Gamma^{o} & =\sum_{i=1}^{(n+1) / 2} \Gamma_{2 i-1}=\Gamma_{1}+\Gamma_{3}+\cdots+\Gamma_{n} .
\end{aligned}
$$

Observe that $\left.\Gamma_{i}\right|_{S_{n-1}}=\Gamma_{i}^{n-1}+\Gamma_{i-1}^{n-1}$ provided $1<i<n$, and $\left.\Gamma_{1}\right|_{S_{n-1}}=\Gamma_{1}^{n-1}$, $\left.\Gamma_{n}\right|_{S_{n-1}}=\Gamma_{n-1}^{n-1}$. Therefore,

$$
\left.\Gamma^{e}\right|_{S_{n-1}}=\Gamma_{1}^{n-1}+\cdots+\Gamma_{n-1}^{n-1}=\left.\Gamma^{o}\right|_{S_{n-1}} .
$$

As $\Gamma=\Gamma^{e}+\Gamma^{o}$, we have $\Gamma^{e}(1)=\Gamma^{o}(1)=\Gamma(1) / 2=2^{n-2}$.

Proposition 6.9. Suppose that $n$ is odd. Then

(a) $\Gamma^{e}$ and $\Gamma^{o}$ are 2-vanishing characters of $S_{n}$;

(b) $\Gamma^{e}$ is Steinberg-like if and only if $n=2^{k}+1$ for some integer $k>0$.

Proof. (a) Let $g \in S_{n}$ be of even order and $g=c h$, where $c$ is a cycle of even size $m$. By Lemma 6.6,

$$
\begin{aligned}
\Gamma^{e}(g) & =\sum_{i=1}^{(n-1) / 2} \Gamma_{n-2 i+1}(g) \\
& =\sum_{i=1}^{(n-1-m) / 2} \Gamma_{n-m-2 i+1}^{n-m}(h)-\sum_{i=(m+2) / 2}^{(n-1) / 2} \Gamma_{n-2 i+1}^{n-m}(h),
\end{aligned}
$$

as $\gamma_{n-m-2 i+1}^{n-m}$ is a proper diagram only for $i<\frac{n-m}{2}$ and $\gamma_{n-2 i+1}^{n-m}$ is a proper diagram only for $i \geq \frac{m+2}{2}$. Set $k=i-\frac{m}{2}$. So the second sum can be written as $\sum_{k=1}^{(n-1-m) / 2} \Gamma_{n-m-2 k+1}^{n-m}(h)$, whence $\Gamma^{e}(g)=0$. 
Similarly,

$$
\begin{aligned}
\Gamma^{o}(g) & =\sum_{i=1}^{(n+1) / 2} \Gamma_{n-2 i+2}(g) \\
& =\sum_{i=1}^{(n-1-m) / 2} \Gamma_{n-m-2 i+2}^{n-m}(h)-\sum_{i=(m+2) / 2}^{(n-1) / 2} \Gamma_{n-2 i+2}^{n-m}(h),
\end{aligned}
$$

as $\gamma_{n-m-2 i+1}^{n-m}$ is a proper diagram only for $i \leq \frac{n-m-1}{2}$ and $\gamma_{n-2 i+2}^{n-m}$ is a proper diagram only for $i \geq \frac{m+2}{2}$. Set $k=i-\frac{m}{2}$. Then the second sum can be written as $\sum_{k=1}^{(n-1-m) / 2} \Gamma_{n-m-2 k+2}^{n-m}(h)$. So $\Gamma^{o}(g)=0$ as well.

(b) If $n=2^{k}+1$, then $\left|S_{n}\right|_{2}=\left|S_{n-1}\right|_{2}=2^{n-2}$ (see the proof of Proposition $6.7(\mathrm{~b}))$. By the above, $\Gamma^{e}(1)=\Gamma^{o}(1)=2^{n-2}$, so both $\Gamma^{e}$ and $\Gamma^{o}$ are Steinberg-like. If $n-1$ is not a 2-power, then $\left|S_{n}\right|_{2}=\left|S_{n-1}\right|_{2}<2^{n-2}$ by Proposition 6.7 (b).

Let $n$ be odd. Then $\left.\Gamma_{i}\right|_{A_{n}}=\left.\Gamma_{n+1-i}\right|_{A_{n}}$ is irreducible for $i \neq \frac{n+1}{2}$, whereas $\left.\Gamma_{(n+1) / 2}\right|_{A_{n}}$ is the sum of two irreducible constituents, denoted by $\Gamma_{(n+1) / 2}^{+}$and $\Gamma_{(n+1) / 2}^{-}$. If $n=4 l+1$, then set

$$
\begin{aligned}
\Gamma^{e a} & =\left.\sum_{i=1}^{(n-1) / 4} \Gamma_{2 i}\right|_{A_{n}}=\left.\left(\Gamma_{2}+\Gamma_{4}+\cdots+\Gamma_{(n-1) / 2}\right)\right|_{A_{n}}, \\
\Gamma^{o \pm} & =\Gamma_{(n+1) / 2}^{ \pm}+\left.\sum_{i=1}^{(n-1) / 4} \Gamma_{2 i-1}\right|_{A_{n}} \\
& =\left.\left(\Gamma_{1}+\Gamma_{3}+\cdots+\Gamma_{(n-3) / 2}\right)\right|_{A_{n}}+\Gamma_{(n+1) / 2}^{ \pm},
\end{aligned}
$$

while, for $n=4 l+3$, we set

$$
\begin{aligned}
\Gamma^{o a} & =\left.\sum_{i=1}^{(n+1) / 4} \Gamma_{2 i-1}\right|_{A_{n}}=\left.\left(\Gamma_{1}+\Gamma_{3}+\cdots+\Gamma_{(n-1) / 2}\right)\right|_{A_{n}}, \\
\Gamma^{e \pm} & =\Gamma_{(n+1) / 2}^{ \pm}+\left.\sum_{i=1}^{(n-3) / 4} \Gamma_{2 i}\right|_{A_{n}} \\
& =\left.\left(\Gamma_{2}+\Gamma_{4}+\cdots+\Gamma_{(n-3) / 2}\right)\right|_{A_{n}}+\Gamma_{(n+1) / 2}^{ \pm} .
\end{aligned}
$$

Corollary 6.10. The following statements hold.

(a) Let $n=4 l+1$. Then $\Gamma^{e a}, \Gamma^{o+}$ and $\Gamma^{o+}$ are 2-vanishing characters of $A_{n}$. If $n=2^{k}+1$, then they are Steinberg-like characters.

(b) Let $n=4 l+3>3$. Then $\Gamma^{e+}, \Gamma^{e-}$ and $\Gamma^{o a}$ are 2-vanishing characters of $A_{n}$. None of them is Steinberg-like. 
Proof. Let $g \in A_{n}$ be of even order.

(a) Let $i \neq \frac{n+1}{2}$. Then $\Gamma_{i}$ remains irreducible under restriction to $A_{n}$. As $\Gamma_{i}$ and $\Gamma_{n-i+1}$ coincide under restriction to $A_{n}$, it follows that $\left.\Gamma^{e}\right|_{A_{n}}=2 \Gamma^{e a}$, and hence $\Gamma^{e a}$ is a 2-vanishing character. As $\Gamma^{e a}(1)=2^{n-3}=\left|A_{n}\right|_{2}$, this is Steinberg-like for $n=2^{k}+1$.

Observe that

$$
\Gamma_{(n+1) / 2}^{+}(g)=\Gamma_{(n+1) / 2}^{-}(g), \quad \Gamma_{(n+1) / 2}^{+}(g)+\Gamma_{(n+1) / 2}^{-}(g)=\Gamma_{(n+1) / 2}(g) .
$$

It follows that $\Gamma^{o+}(g)=\Gamma^{o-}(g)=\Gamma^{o}(g) / 2$, and thus $\Gamma^{o+}(g)=\Gamma^{o-}(g)=0$ by Proposition 6.9. Therefore, $\Gamma^{o+}$ and $\Gamma^{o+}$ are 2-vanishing characters of $A_{n}$. In addition, suppose that $n=2^{k}+1$. Then $\Gamma^{o+}(1)=\Gamma^{o-}(1)=\Gamma^{o}(1) / 2=\left|A_{n}\right|_{2}$, so both $\Gamma^{o+}$ and $\Gamma^{o-}$ are Steinberg-like.

(b) Let $i \neq \frac{n+1}{2}$. Then, as above, it follows that $\left.\Gamma^{o}\right|_{A_{n}}=2 \Gamma^{o a}$, and hence $\Gamma^{o a}$ is a 2 -vanishing character. In addition, $\Gamma^{o a}(1)=\Gamma^{o}(1) / 2=2^{n-3}$. As here we never have $n=2^{k}+1, \Gamma^{o a}$ is not Steinberg-like.

Consider $\Gamma^{e \pm}$. Observe that

$$
\Gamma_{(n+1) / 2}^{+}(g)=\Gamma_{(n+1) / 2}^{-}(g), \quad \Gamma_{(n+1) / 2}^{+}(g)+\Gamma_{(n+1) / 2}^{-}(g)=\Gamma_{(n+1) / 2}(g) .
$$

It follows that $\Gamma^{e+}(g)=\Gamma^{e-}(g)=\Gamma^{e}(g) / 2$, and so $\Gamma^{e+}(g)=\Gamma^{e-}(g)=0$ by Proposition 6.9. Therefore, $\Gamma^{e+}$ and $\Gamma^{e-}$ are 2-vanishing characters of $A_{n}$ but not Steinberg-like.

Lemma 6.11. Let $2 \leq n \leq 12$. Then, in addition to the character $\Gamma$ when $n=2^{k}$, and the characters $\Gamma^{e}$ and $\Gamma^{o}$ when $n=2^{k}+1$, the only Steinberg-like characters of $S_{n}$ are:

(a) if $n=4$, the sum of all non-linear irreducible characters;

(b) if $n=6$, the irreducible character of degree 16;

(c) if $n=8$, the sum of the two irreducible characters of degree 64 .

Proof. For $n \leq 6$, this is easily checked from the known character tables. For $n=8$, we use a computer program to go through all possibilities. For $S_{10}$, one checks that no character exists with the right restriction to $S_{8} \times S_{2}$, and similarly for $S_{12}$, one considers the restriction to $S_{8} \times S_{4}$. Finally, the cases $n \in\{7,9,11\}$ are treated by restricting to $S_{n-1}$.

Theorem 6.12. Suppose that the only Steinberg-like character of $S_{2^{k}}, k \geq 4$, for $p=2$ is the one constructed in Proposition 6.7. Then $A_{n}$ does not have Steinberglike characters for $p=2$ for $n \geq 13$ unless $n$ or $n-1$ is a 2-power. In the latter case, the only Steinberg-like characters are those listed in Proposition 6.9. 
Proof. Let $\psi$ be a Steinberg-like character for $p=2$ of $A_{n}$, with $n \geq 10$. Then $\chi:=\psi^{S_{n}}$ is Steinberg-like for $S_{n}$. We argue by induction on $n$ that $S_{n}$ does not have a Steinberg like character unless $n$ or $n-1$ is a power of 2 .

Assume that $n$ is not a power of 2 , and write $n=2^{a_{1}}+\cdots+2^{a_{r}}$ for distinct exponents $a_{1}, \ldots, a_{r}>0$. By Lemma 6.11 , we may assume $n \neq 12$, so one of the summands, say $2^{a_{1}}$, is different from 4 and 8 . Then the Young subgroup $Y=Y_{1} \times Y_{2}:=S_{2^{a_{1}}} \times S_{n-2^{a_{1}}}$ of $S_{n}$ contains a Sylow 2-subgroup, so $\left.\chi\right|_{Y}$ is Steinberg-like. Then, by Lemma 2.7, we have $\left.\chi\right|_{Y}=\sum_{i} \eta_{i} \sigma_{i}$, where $\eta_{i} \in \operatorname{Irr}\left(Y_{1}\right)$ are all distinct, the $\sigma_{i}$ are 2-vanishing characters of $Y_{2}$, and $\chi_{1}:=\sum_{i} l_{p}\left(\sigma_{i}\right) \eta_{i}$ is a 2-vanishing character of $Y_{1}$ with $l_{2}\left(\chi_{1}\right)=l_{2}(\chi)=1$. Thus, by assumption, $\chi_{1}$ is the character $\Gamma$ from Proposition 6.7. In particular, $\chi_{1}$ is multiplicity-free, and so $l_{p}\left(\sigma_{i}\right)=1$ for all $i$. So the $\sigma_{i}$ are Steinberg-like as well. This is not possible unless $n-2^{a_{1}}$ is a 2-power as well.

In the latter case, by Lemma 6.11, we conclude that $n \geq 17$. The above argument shows that $\left.\chi\right|_{Y}=\Gamma^{(1)} \otimes \Gamma^{(2)}$, with $\Gamma^{(j)}$ a Steinberg-like character of $Y_{j}$. So, in particular, $\left.\chi\right|_{Y}$ and hence also $\chi$ is multiplicity-free. By possibly interchanging $a_{1}, a_{2}$, we may assume that $2^{a_{1}}>8$. Now consider $\left.\chi\right|_{Y_{1}}=\left|Y_{2}\right|_{2} \Gamma^{(1)}$, a sum of hooks. By the branching rule, any non-hook character of $S_{m}$ restricted to $S_{m-1}$ contains a non-hook character (except when $m=4$, which is excluded here). Thus, inductively, $\chi$ cannot contain any non-hook constituent. This, in turn, means that all constituents of $\left.\chi\right|_{Y_{2}}$ are hooks and thus by induction that $\left.\chi\right|_{Y_{2}}=\left|Y_{1}\right|_{2} \Gamma^{(2)}$. Now observe that by the Littlewood-Richardson rule [9, Lemma 21.3], $\left.\Gamma_{i}\right|_{Y}$ contains $\Gamma_{j}^{(1)} \otimes \Gamma_{l}^{(2)}$ if and only if $j+l \in\{i, i+1\}$. Thus, on the one hand, $\left.\Gamma_{i}\right|_{Y}$ and $\left.\Gamma_{i+1}\right|_{Y}$ have a common constituent, and so at most every second hook character occurs in $\chi$. On the other hand, every second hook must indeed occur. Thus, either $\chi=\Gamma^{e}$ or $\chi=\Gamma^{o}$ as defined above. If $n=2^{a_{1}}+1$, then our claim follows from Proposition 6.9; otherwise, the degree of $\chi$ is larger than $\left|S_{n}\right|_{2}$.

\subsection{Projective characters for $p=2$}

Lemma 6.13. Let $p=2$. Then $A_{n}$ has a projective character of degree $\left|A_{n}\right|_{2}$ if and only if $S_{n}$ has a projective character of degree $\left|S_{n}\right|_{2}$.

Proof. This follows from Lemma 2.13.

Theorem 6.14. Let $p=2$ and $G=A_{n}$ or $S_{n}$ for $n>4$. Then $G$ has no reducible projective character of degree $|G|_{2}$.

Proof. One can inspect the decomposition matrix modulo 2 of $G=A_{n}$ for $n \leq 9$ to observe that $G$ has no projective character of degree $|G|_{2}$. Analysing the character table of $G=A_{n}$ for $9<n \leq 15$, one observes that $G$ has no $\mathrm{Syl}_{2}$-regular characters, and hence no PIM of dimension $|G|_{2}$. 
One can inspect the decomposition matrix of $G=A_{9}$ to observe that the minimal dimension of a PIM is 320. Analysing the character table of $G=A_{n}$ for $9<n \leq 15$, one observes that $G$ has no $\mathrm{Syl}_{2}$-regular characters, and hence no PIM of dimension $|G|_{2}$.

Let $n=16$. Using the known character table of $A_{16}$, one finds that there is a unique $\mathrm{Syl}_{2}$-regular character, viz. the character $\Gamma^{0}$; it is multiplicity-free with constituents of degrees 1, 15, 105, 455, 1365, 3003, 5005, 6435, and Steinberglike. Recall that the principal PIM is the only one that has $1_{G}$ as a constituent. However, by [13], the principal PIM is not of degree $|G|_{2}$.

Let $n=2^{k}$, where $k>4$. Then $G$ has a subgroup $Y$ such that $Y / N \cong A_{16}$ for some normal 2-subgroup $N$ and $|Y|_{2}=|G|_{2}$. Indeed, let $P_{1}, \ldots, P_{16}$ be a partition of $\{1, \ldots, n\}$ with all parts of size $\frac{n}{16}$. If $G=S_{n}$, then $N$ is the direct product of 16 copies of a Sylow 2-subgroup of $S_{n / 16}$. If $G=A_{n}$, then we take $N \cap A_{n}$ for the subgroup in question. Then $Y$ is a semidirect product of $N$ with $S_{16}$. The latter permutes $P_{1}, \ldots, P_{16}$ in the natural way. One easily observes that $|G: Y|$ is odd.

If $\Phi$ is a PIM of degree $|G|_{2}=|Y|_{2}$, then so is $\left.\Phi\right|_{Y}$. By [23, Lemma 3.8], the generalised restriction $r_{Y / N}^{G}(\Phi)$ of $\Phi$ is a PIM of dimension $|Y / N|_{2}$. Such a PIM does not exist as we have just seen.

Let $n>16$ be not a 2-power, and write $n=2^{k}+m$, where $m<2^{k}$. Further, let $X=X_{1} \times X_{2} \leq S_{n}$, where $X_{1} \cong S_{2^{k}}$ and $X_{2} \cong S_{m}$. Then the index $\left|S_{n}: X\right|$ is odd, so $\left.\Phi\right|_{X}$ is a PIM of degree $|X|_{2}$. Therefore, $\left.\Phi\right|_{X}$ is a direct product $\Phi_{1} \times \Phi_{2}$, where $\Phi_{i}$ is a PIM for $X_{i}$ for $i=1,2$. Obviously, $\operatorname{dim} \Phi_{i}=\left|X_{i}\right|_{2}$. This is a contradiction as $X_{1}$ has no PIM of degree $|X|_{1}$. For $G=A_{n}$, the result follows from Lemma 6.13.

\section{Exceptional groups of Lie type}

Theorem 7.1. Let $G$ be a simple group of Lie type which is not classical. Then $G$ does not have a $\mathrm{Syl}_{p}$-regular character in non-defining characteristic, except for the group $G={ }^{2} F_{4}(2)^{\prime}$ which has two reducible $\mathrm{Syl}_{3}$-regular characters and two irreducible Steinberg-like characters for $p=3$.

Proof. As in the proof of [13, Theorem 4.1], we compare the maximal order of a Sylow $p$-subgroup of $G$, which is bounded above by the order of the normaliser of a maximal torus, with the smallest irreducible character degrees (given for example in [20, Table I]). This shows that, except for very small $q$, there cannot be any examples of $\mathrm{Syl}_{p}$-regular characters. A closer inspection of the finitely many remaining cases shows that $G={ }^{2} F_{4}(2)^{\prime}$ has two reducible $S_{1} l_{3}$-regular characters and two irreducible Steinberg-like character for $p=3$, but no further cases arise. 


\section{Groups of Lie type in their defining characteristic}

It was shown in [17] that simple groups of Lie type of sufficiently large rank do not have Steinberg-like characters with respect to the defining characteristic apart from the (irreducible) Steinberg character. More precisely, the Steinberg-like characters were classified except for groups of types $B_{n}$ with $3 \leq n \leq 5$ and $D_{n}$ with $n=4,5$.

Here we deal with the remaining cases.

Proposition 8.1. Let $G=\operatorname{Spin}_{2 n+1}(q), n \in\{3,4,5\}$, with $q=p^{f}$ odd. Then $G$ has no reducible Steinberg-like character with respect to $p$.

Proof. We freely use results and methods from [17]. First assume that $n=3$. According to [17, Proposition 6.2], it suffices to consider a group $H$ (coming from an algebraic group with connected centre) such that $[H, H]=\operatorname{Spin}_{7}(q)$. Let $\chi$ be a reducible Steinberg-like character of $H$. Then $\chi$ has a linear constituent by [17, Theorem 8.6]. Multiplying by the inverse of that character, we may assume that the trivial character occurs in $\chi$ (exactly once). By [17, Lemma 2.1], then all constituents of $\chi$ belong to the principal $p$-block, so we may in fact replace $H$ by $H / Z(H)$, that is, we may assume that $H$ is of adjoint type.

Let $P \leq H$ be a parabolic subgroup of $H$ and $U=O_{p}(P)$. By Lemma 2.5 (c), the Harish-Chandra restriction $r_{L}^{H}(\chi)$ is a Steinberg-like character of $L=P / U$. We will show that there is no possibility for $\chi$ compatible with Harish-Chandra restriction to all Levi subgroups.

Clearly, $r_{L}^{H}(\chi)$ also contains the trivial character, so is again reducible. The reducible Steinberg-like characters of all proper Levi subgroups of $H$ are known by [17, Lemmas 7.4 and 7.8]. In particular, we must have $7 \mid(q+1)$ and, for $L$ of type $A_{2}$, we have $r_{L}^{H}(\chi)=1_{L}+\mu$ with $\mu \in \operatorname{Irr}(L)$ of degree $q^{3}-1$. Thus, $\mu$ lies in the Lusztig series of a regular semisimple element $s \in L^{*}$ (the dual group of $L$ ) with centraliser a maximal torus of order $\left(q^{2}-1\right)(q-1)$. Thus, $\chi$ has to contain a constituent $\psi_{1}$ lying in the Lusztig series of $s$. It is easily seen that the centraliser of $s$ in $G^{*}$ is either a maximal torus, or of type $A_{1}(q) .\left(q^{2}-1\right)$. Correspondingly,

$$
\psi_{1}(1) \in\left\{\left(q^{6}-1\right)\left(q^{2}+1\right)(q+1),\left(q^{6}-1\right)\left(q^{2}+1\right), q\left(q^{6}-1\right)\left(q^{2}+1\right)\right\} .
$$

But the first and the last are bigger than $q^{9}$, so $\psi_{1}(1)=\left(q^{6}-1\right)\left(q^{2}+1\right)$. Now if $\chi$ contains any other constituent apart from $1_{G}$ in the principal series, then its generalised restriction to $L$ is non-zero, contradicting $r_{L}^{H}(\chi)=1_{L}+\mu$.

Next, the Harish-Chandra restriction to a Levi subgroup $L$ of type $B_{2}$ has the form

$$
r_{L}^{H}(\chi)=1_{L}+v_{1}+v_{2}+v_{3}
$$


with $v_{1}(1)=(q-1)^{2}\left(q^{2}+1\right)$ and $v_{2}(1)=v_{3}(1)=(q-1)\left(q^{2}+1\right)$. In particular, $v_{1}$ lies in the Lusztig series of a regular semisimple element $t \in L^{*}$ (of order 7 dividing $q+1)$ with centraliser a maximal torus of order $\left(q^{2}-1\right)(q+1)$. The centraliser of $t$ in $G^{*}$ then either is the same maximal torus, or of type $A_{1}(q) \cdot(q+1)^{2}$. Correspondingly, $\chi$ has a constituent $\psi_{2}$ in the Lusztig series of $t$ of degree

$$
\begin{aligned}
& d_{1}:=\frac{\left(q^{6}-1\right)\left(q^{2}+1\right)(q-1)}{q+1}, \\
& d_{2}:=\frac{q\left(q^{6}-1\right)\left(q^{2}+1\right)(q-1)}{q+1} \text { or }\left(q^{6}-1\right)\left(q^{2}+1\right)(q-1) .
\end{aligned}
$$

The last one is larger than $q^{9}-1-\psi_{1}(1)$, so $\psi_{2}(1) \in\left\{d_{1}, d_{2}\right\}$. Furthermore, by [17, Lemma 3.1], $\chi$ contains at least one regular constituent. This is either $\psi_{2}$ of degree $d_{2}$, or, if $\psi_{2}(1)=d_{1}$, then one can check from the known list of character degrees of $H$ (which can be found at [12]) that the only regular character $\psi_{3}$ of small enough degree has degree $d_{3}:=\frac{\left(q^{6}-1\right)\left(q^{2}+1\right)(q-1)^{2}}{q+1}$. Observe that $d_{2}=d_{1}+d_{3}$. So the sum of remaining character degrees is

$$
d:=q^{9}-1-\psi_{1}(1)-d_{2}=q\left(q^{3}-1\right)\left(q^{4}-3 q^{3}+3 q^{2}-3 q+1\right) .
$$

Now note that $\chi$ cannot have further unipotent constituents since they would lead to unipotent constituents of $r_{L}^{H}(\chi)$ (as $H$ has no cuspidal unipotent characters). It turns out that all remaining candidates except for one of degree

$$
\lambda(1)=\left(q^{3}-1\right)\left(q^{2}+1\right)(q-1)
$$

have degree divisible by $q^{2}-q+1$. Now we have $\lambda(1) \equiv 2\left(\bmod q^{2}-q+1\right)$, while $d \equiv-2\left(\bmod q^{2}-q+1\right)$. It follows that $\lambda$ would have to occur at least $q^{2}-q$ times in $\chi$. As $\left(q^{2}-q\right) \lambda(1)>d$, this is not possible. This contradiction concludes the proof for the case $n=3$.

The cases of $\operatorname{Spin}_{9}(q)$ and $\operatorname{Spin}_{11}(q)$ now follow from the previous one by application of the inductive argument in the proof of [17, Theorem 10.1].

Proposition 8.2. Let $G=\operatorname{Spin}_{2 n}^{+}(q), n \in\{4,5\}$ and $q=p^{f}$. Then $G$ has no reducible Steinberg-like character with respect to $p$.

Proof. First consider the case $n=4$. As in the previous proof, by [17, Proposition 6.2 and Lemma 2.1], we may work with $H$ of adjoint type. Let $\chi$ be a reducible Steinberg-like character of $H$. Then $\chi$ contains $1_{H}$ by [17, Theorem 8.6] 
and hence so does its Harish-Chandra restriction $r_{L}^{H}(\chi)$ to a Levi subgroup of type $A_{3}$. Then, by [17, Lemma 9.1], we have $q \equiv-1(\bmod 3)$ and

$$
r_{L}^{H}(\chi)=1_{L}+\mu_{1}+\mu_{2}+\mu_{3}
$$

with $\mu_{1}$ a cuspidal character labelled by a regular element $s \in L^{*}$ in a torus of order $q^{4}-1$, of order dividing $\left(q^{2}+1\right)(q+1)$ in $L^{*} / Z\left(L^{*}\right)$. But then $s$ is also regular in $H^{*}$, that is, $\chi$ has a constituent $\psi$ of degree $\left(q^{6}-1\right)\left(q^{4}-1\right)\left(q^{2}-1\right)$. This holds for all three conjugacy classes of Levi subgroups of type $A_{3}$. Comparison of degrees shows that this is not possible. The case of $\operatorname{Spin}_{10}(q)$ again follows from the previous one by application of the argument in the proof of [17, Theorem 10.1].

\section{Classical groups of large rank}

As an application of results obtained in Section 6, we show here that classical groups of large rank have no Steinberg-like character for $p>2$, provided $p$ is not the defining characteristic of $G$. Throughout, $p$ is an odd prime not dividing $q$, and we set $e:=e_{p}(q)$, the order of $q$ modulo $p$. We first illustrate our method on the groups $\mathrm{GL}_{n}(q)$.

Lemma 9.1. Let $G=\mathrm{GL}_{n}(q), p>2$, and let $S$ be a Sylow $p$-subgroup of $G$.

(a) Write $n=m e+m^{\prime}$, where $0 \leq m^{\prime}<e$. Then there exist subgroups

$$
U \leq S \leq N \leq G
$$

where $U$ is an abelian normal p-subgroup of $N$ and $N / U \cong A_{m}$.

(b) If $m>\max \{6, p+1\}$ or $m^{\prime}>0$, then $G$ has no Steinberg-like character.

Proof. (a) See [21].

(b) If $m^{\prime}>0$, then $G$ contains a subgroup $X$ such that $X \cong \mathrm{GL}_{m^{\prime}}(q)$ and $C_{G}(X)$ contains a Sylow $p$-subgroup of $G$. As $X$ is a $p^{\prime}$-group, the result follows from Lemma 2.3. Let $m^{\prime}=0$. Suppose the contrary, and let $\chi$ be a Steinberg-like character of $G$. By Lemma 2.5, $A_{m}$ must have a Steinberg-like character. However, this is false by Theorem 6.4.

For other classical groups, the argument is similar, but involves more technical details. Let $d=e_{p}(-q)$ be the order of $-q$ modulo $p$, equivalently, $d=2 e$ if $e$ is odd, $d=\frac{e}{2}$ if $e \equiv 2(\bmod 4)$, and $d=e$ if $4 \mid e$. So $d=1$ if and only if $e=2$, equivalently, $p \mid(q+1)$. Note that $e=2 e_{p}\left(q^{2}\right)$ if $e$ is even. 
Lemma 9.2 ([21]). Let $G=\mathrm{GU}_{n}(q)$ and $p>2$. Then the Sylow $p$-subgroups of $G$ are isomorphic to those of $H$, where

$$
H \cong \begin{cases}\operatorname{GL}_{\lfloor n / 2\rfloor}(q) & \text { if e is odd } \\ \operatorname{GL}_{\lfloor n / 2\rfloor}\left(q^{2}\right) & \text { if } 4 \mid e, \\ \operatorname{GL}_{n}\left(q^{2}\right) & \text { if } e \equiv 2(\bmod 4)\end{cases}
$$

Lemma 9.3. Let $G=\mathrm{GU}_{n}(q), p>2$, and let $S$ be a Sylow p-subgroup of $G$. Suppose that $e \equiv 2(\bmod 4)$, equivalently, $d$ is odd.

(a) Write $n=m d+m^{\prime}$, where $0 \leq m^{\prime}<d$. Then there exist subgroups

$$
U \leq S \leq N \leq G
$$

where $U$ is an abelian normal p-subgroup of $N$ and $N / U \cong A_{m}$.

(b) If $m>\max \{6, p+1\}$ or $m^{\prime}>0$, then $G$ has no Steinberg-like character.

Proof. (a) Suppose first that $e=2$. Let $V$ be the natural $\mathbb{F}_{q^{2}} H$-module. Then $V$ is a direct sum $\oplus_{i=1}^{n} V_{i}$, where $V_{i}$ 's are non-degenerate subspaces of dimension 1 . Let $X$ be the stabiliser of this decomposition, that is, $X=\left\{x \in G \mid x V_{i}=V_{j}\right.$ for some $j=j(x) \in\{1, \ldots, n\}\}$. Then $X \cong X_{1} \cdot S_{n}$ (a semidirect product), where $X_{1} \cong\left(\mathrm{GU}_{1}(q) \times \cdots \times \mathrm{GU}_{1}(q)\right)$ ( $n$ factors). Let $U$ be the Sylow $p$-subgroup of $X_{1}$. Then $U$ is normal in $X$ and abelian. It is well known that $X$ contains a Sylow $p$-subgroup of $G$. Therefore, $N=U A_{n}$ satisfies the statement.

Let $e>2$. As $d$ is odd, there is an embedding $\mathrm{GU}_{m}\left(q^{d}\right) \rightarrow G U_{m d}(q)$ (see [8, Hilfssatz 1]). Note that $e_{p}\left(q^{d}\right)=2,\left|\mathrm{GU}_{m}\left(q^{d}\right)\right|_{p}=\left|\mathrm{GU}_{m d}(q)\right|_{p}$. As $\operatorname{GU}_{m d}(q)$ is isomorphic to a subgroup of $G$, the result follows.

(b) is similar to the proof of Lemma 9.1 (b).

Lemma 9.4. Let $p>2,2 n=m e$, where $e=e_{p}(q)$ is even, and $X=\operatorname{GU}_{m}\left(q^{e / 2}\right)$.

(a) If $m$ is even (respectively, odd), $X$ is isomorphic to a subgroup of $\mathrm{GO}_{2 n}^{+}(q)$ (respectively, $\mathrm{GO}_{2 n}^{-}(q)$ ).

(b) $X$ is isomorphic to a subgroup of $\mathrm{Sp}_{2 n}(q)$, of $\mathrm{GO}_{2 n+1}(q)$, of $\mathrm{GO}_{2 n+e}^{+}(q)$ as well as of $\mathrm{GO}_{2 n+e}^{-}(q)$.

In addition, $X$ contains a Sylow p-subgroup of the respective group.

Proof. (a) follows from [3, Lemma 6.6] as well as (b) for $\operatorname{Sp}_{2 n}(q)$. The second case in (b) follows from (a) as the groups $\mathrm{GO}_{2 n+1}(q), \mathrm{GO}_{2 n+e}^{+}(q), \mathrm{GO}_{2 n+e}^{-}(q)$ contain subgroups isomorphic to $\mathrm{GO}_{2 n}^{+}(q)$ and $\mathrm{GO}_{2 n}^{-}(q)$.

The additional statement can be read off from the orders of the groups in question. (The cases with $\operatorname{Sp}_{2 n}(q), \mathrm{GO}_{2 n}^{-}(q)$ and $\mathrm{GO}_{2 n+1}(q)$ are considered in [6, Lemmas 3.14 and 3.16]; that of $\mathrm{GO}_{2 n}^{+}(q)$ is similar.) 
Lemma 9.5. Let $p>2$. Let $H$ be one of the following groups:

(1) $H=\mathrm{GU}_{n}(q)$ with $n=m d+m^{\prime}$, where $m^{\prime}<d$;

(2) $H \in\left\{\mathrm{Sp}_{2 n}(q), \mathrm{GO}_{2 n+1}(q), \mathrm{GO}_{2 n}^{+}(q), \mathrm{GO}_{2(n+1)}^{-}(q)\right\}, n=m e+m^{\prime}$, where $e$ is odd and $m^{\prime}<e$;

(3) $H \in\left\{\operatorname{Sp}_{2 n}(q), \mathrm{GO}_{2 n+1}(q)\right\}$ with $2 n=m e+m^{\prime}$ where e is even and $m^{\prime}<e$;

(4) $H=\operatorname{GO}_{2 n}^{ \pm}(q), 2 n=m e+m^{\prime}$, where e is even, $m^{\prime}<e$, and either $m^{\prime}>0$, or $m^{\prime}=0$ and then either $H=\mathrm{GO}_{2 n}^{+}(q), m$ is even, or $H=\mathrm{GO}_{2 n}^{-}(q), m$ is odd;

(5) let e be even, $2 n=(m+1) e, H=\mathrm{GO}_{2 n}^{+}(q), m+1$ is odd, or $H=\mathrm{GO}_{2 n}^{-}(q)$ and $m+1$ is even.

Let $S$ be a Sylow p-subgroup of $H$. Then there exist subgroups $U \leq S \leq P \leq H$, where $U$ is an abelian normal $p$-subgroup of $P$ and $P / U \cong A_{m}$.

Proof. (1) The case $e \equiv 2(\bmod 4)$ is handled in Lemma 9.3. In the remaining cases, the result follows from Lemmas 9.1 and 9.2 as $\operatorname{GL}_{[n / 2]}\left(q^{2}\right)$ is isomorphic to a subgroup of $G$.

(2) By Lemma 9.2, $|H|_{p}=\left|\mathrm{GL}_{n}(q)\right|_{p}$. So the result follows from Lemma 9.1.

(3) This follows from Lemmas 9.4 and 9.1. (Note that $H=\mathrm{GO}_{2 n+1}(q)$ contains subgroups isomorphic to $\mathrm{GO}_{2 n+1}^{+}(q)$ and $\mathrm{GO}_{2 n}^{-}(q)$, and one of them contains a Sylow $p$-subgroup of $H$.)

(4) is similar to (3). Note that if $m^{\prime}>0$, then $H$ contains subgroups isomorphic to $\mathrm{GO}_{m e}^{+}(q)$ and $\mathrm{GO}_{m e}^{-}(q)$, and one of them contains a Sylow $p$-subgroup of $H$.

(5) In this case, a subgroup $X$ of $H$ isomorphic to $\mathrm{GO}_{2 n-e}^{+}(q)$ and $\mathrm{GO}_{2 n-e}^{-}(q)$, respectively, contains a Sylow $p$-subgroup of $H .\left(|H: X|_{p}=1\right.$ is easily checked.) So the result follows from (4).

Our result for alternating groups (Corollary 6.5) implies the following:

Proposition 9.6. Let $p>2$ and $e=e_{p}(q)$. Let $m=\max \{7, p+2\}$. Let $G$ be one of the following groups:

(1) $\operatorname{PSL}_{n}(q)$ and $n \geq e m$,

(2) $\operatorname{PSU}_{n}(q)$ and $n \geq d m$, where $d=e_{p}(-q)$,

(3) $\Omega_{2 n+1}(q), q$ odd, or $\operatorname{PSp}_{2 n}(q), n>1$, and $n \geq$ em if $e$ is odd, otherwise $2 n \geq e m$,

(4) $\mathrm{P} \Omega_{2 n}^{+}(q)$, and $n \geq e m$ if $e$ is odd, otherwise $2 n \geq e m$,

(5) $\mathrm{P} \Omega_{2 n}^{-}(q)$, and $n-1 \geq$ em if e is odd, otherwise $2 n \geq e \mathrm{em}$. 
Then $G$ has no Steinberg-like character. This remains true for any group $H$ such that $G$ is normal in $H / Z(H)$ and $(H / Z(H)) / G$ is abelian.

Proof. Suppose first that $H$ is as in Lemma 9.5. Let $S \in \operatorname{Syl}_{p}(H)$. Then there are subgroups $U \leq S \leq P \leq H$, where $U$ is normal in $P$ and $N / U \cong A_{m}$ with $m \geq m=\max \{7, p+2\}$. So $A_{m}$ is perfect. Let $H_{1}$ be the derived subgroup of $H$. Set $P_{1}=P \cap H_{1}, S_{1}=S \cap H_{1}, U_{1}=U \cap H_{1}$. Then we have $S_{1} \in \operatorname{Syl}_{p}\left(H_{1}\right)$, $U_{1} \leq S_{1} \leq P_{1} \leq H_{1}$ and $P_{1} / U_{1} \cong A_{m}$, as $A_{m}$ is perfect. A similar statement is true for the quotient of $H_{1}$ by a central subgroup. Then the result follows from Theorem 6.4 using Lemma 2.5.

\section{Minimal characters and Sylow $p$-subgroups, $p>2$}

In this section, we show that if $p>2$ and $G$ is a simple classical group not satisfying the assumptions in Proposition 9.6, $p$ is not the defining characteristic of $G$ and a Sylow $p$-subgroup $S$ of $G$ is not cyclic, then $G$ has no $S_{1} p_{p}$-regular character and hence no Steinberg-like character. Observe that $S$ is cyclic if and only if $m=1$, and abelian if and only if $m<p$, where $m$ is as in Lemma 9.5. The case where $S$ is cyclic has been dealt with in Section 4 .

For a group $G$, let $\mu_{0}(G)=1<\mu_{1}(G)<\mu_{2}(G)<\cdots$ denote the sequence of integers such that, for $i>0, G$ has an irreducible character of degree $\mu_{i}(G)$ and no irreducible character $\rho$ with $\mu_{i-1}(G)<\rho(1)<\mu_{i}(G)$. For universal covering groups of finite classical groups, the values $\mu_{1}(G), \mu_{2}(G), \mu_{3}(G)$ were determined in [20]. In our analysis below, these three values play a significant role, but mainly for classical centreless groups $G$ such as $\operatorname{PGL}_{n}(q), \operatorname{PGU}_{n}(q), \operatorname{PSp}_{2 n}(q)$, $\mathrm{P} \Omega_{2 n}^{ \pm}(q)$ and $\Omega_{2 n+1}(q)$. For these groups, mainly for $2 e \leq n \leq p e$ with $p>2$, we observe that $|G|_{p}<\mu_{3}(G)$ and sometimes $|G|_{p}<\mu_{1}(G)$. In the latter case, it is immediate to conclude that $G$ has no $\operatorname{Syl}_{p}$-regular character; in the other cases, we observe that there exists an element $g \in G$ of order $p$ such that $\rho(g)>0$ for each irreducible character $\rho$ of degree at most $\mu_{2}(G)$. For $n>p e$, we use a different method.

Recall that $e=e_{p}(q)$ denotes the minimal integer $i>0$ such that $q^{i}-1$ is divisible by $p$.

\subsection{The groups $\mathrm{GL}_{n}(q), n \geq 2 e$}

Set $d_{n}=\frac{q^{n}-1}{q-1}$. Let $G=\operatorname{SL}_{n}(q)$. The minimal degrees of projective irreducible representations of $\operatorname{PSL}_{n}(q)$ are given in [20, Table IV]. Table 1 is obtained from this by omitting the representations that are not realisable as ordinary representations of $\operatorname{SL}_{n}(q)$. 


\begin{tabular}{llll}
\hline$n, q$ & $\mu_{1}(G)$ & $\mu_{2}(G)$ & $\mu_{3}(G)$ \\
\hline$n=3, q>2$ & $d_{3}-1$ & $d_{3}$ & $\frac{\left(q^{2}-1\right)(q-1)}{(3, q-1)}$ \\
$n=4, q>3$ & $d_{4}-1$ & $d_{4}$ & $\frac{\left(q^{3}-1\right)(q-1)}{(2, q-1)}$ \\
$n=4, q=3$ & 26 & 39 & 52 \\
$n>4, q>2,(n, q) \neq(6,3)$ & $d_{n}-1$ & $d_{n}$ & $\frac{d_{n}\left(q^{n-1}-q^{2}\right)}{q^{2}-1}$ \\
$n>4, q=2, n \neq 6$ & $d_{n}-1$ & $\frac{d_{n}\left(2^{n-1}-4\right)}{3}$ & $\frac{d_{n} d_{n-1}}{3}$ \\
$n=6, q=2$ & 62 & 217 & 588 \\
$n=6, q=3$ & 363 & 364 & 6318 \\
\hline
\end{tabular}

Table 1. Minimal degrees of irreducible characters of $\mathrm{SL}_{n}(q)$

Lemma 10.1. Let $p>2, e>1$ and $G=\mathrm{GL}_{e n}(q)$. Suppose that $1<n \leq p$, and if $q=2$, suppose that either $n<p$ or $p<2^{e}-1$. Then $|G|_{p}<\mu_{1}(G)$, and $G$ has no $\mathrm{Syl}_{p}$-regular character.

Proof. If $n<p$, then we have $|G|_{p} \leq \frac{\left(q^{e}-1\right)^{n}}{(q-1)^{n}}$ (as $p$ is coprime to $q-1$ ) and $\mu_{1}(G)=\frac{q^{e n}-q}{q-1}$. So the statement is obvious in this case.

Let $n=\stackrel{q-1}{p}$. If $q>2$, then $|G|_{p} \leq \frac{p\left(q^{e}-1\right)^{p}}{(q-1)^{p}}$, while $\mu_{1}(G)=\frac{q^{e p}-q}{q-1}$. (As $p>2$, the exceptions in Table 1 can be ignored, except for $e=3$ and $(n, q)=(6,2)$ or $(6,3)$; these two cases are trivial.) We have $p\left(q^{e}-1\right)^{p}<(q-1)^{p-1}\left(q^{e p}-q\right)$ as $p<(q-1)^{p-1}$ for $q>2$ and $\left(q^{e}-1\right)^{p}<q^{e p}-q$.

If $q=2$ and $p<2^{e}-1$, then we have $p \leq \frac{2^{e}-1}{3}$, and $|S| \leq \frac{p\left(2^{e}-1\right)^{p}}{3^{p}}$ is less than $2^{e p}-2=\mu_{1}(G)$ as $p<3^{p}$.

Remark 10.2. Lemma 10.1 does not extend to the case $q=2$ with $n=p=2^{e}-1$ as then

$$
|G|_{p}=p\left(2^{e}-1\right)^{p}=p^{p+1}>(p+1)^{p}-2=2^{e p}-2=\mu_{1}(G) .
$$

So the case $e>1$ leaves us with $q=2$, which we deal with next.

Lemma 10.3. Let $e>1$ and $G=\mathrm{GL}_{e p}(2)$. Then $|G|_{p}<\mu_{2}(G)$, and $G$ has no $\mathrm{Syl}_{p}$-regular character.

Proof. We have $|G|_{p} \leq p\left(2^{e}-1\right)^{p}$. By Table 1, we have

$$
\mu_{1}(G)=2^{e p}-2 \quad \text { and } \quad \mu_{2}(G)=\frac{\left(2^{e p}-1\right)\left(2^{e p-1}-4\right)}{3}>|G|_{p},
$$


or $e p=3,4,6$. As $p$ is odd and $e>1$, we have $e p \neq 3,4$, so, in the exceptional cases, $e=2, p=3$, where $|G|_{p}=81<\mu_{2}(G)=217$.

Let $\pi$ be the permutation character of $G$ associated with the action of $G$ on the non-zero vectors of the natural $\mathbb{F}_{2} G$-module. Then $\pi=\tau+1_{G}$, where $\tau$ is a character of $G$ of degree $\tau(1)=2^{e p}-2$. There is a unique irreducible character of $G$ of degree $2^{e p}-2$ (see [20, Table IV]), and hence it coincides with $\tau$. Let $\chi$ be a Syl $p$-regular character of $G$. As $\chi(1)=|G|_{p}$, it follows that the irreducible constituents of $\chi$ are either $1_{G}$ or $\tau$. As $\chi(g)=0$ for every $p$-element $g \in G$, we get a contradiction as soon as we show that $\tau(g)>0$ for some $p$-element $g \in G$. This is equivalent to showing that $\pi(g)>1$. This can be easily verified.

Lemma 10.4. Suppose that $p>2, p \mid(q-1)$, and let $\mathrm{SL}_{n}(q) \leq G \leq \mathrm{GL}_{n}(q)$ for $2<n<p$. Then $G$ has no $\mathrm{Syl}_{p}$-regular character.

Proof. Let $G_{1}=G / Z(G)$. Then

$$
\left|G_{1}\right|_{p} \leq|q-1|_{p}^{n-1} \quad \text { and } \quad \mu_{1}\left(G_{1}\right)=\frac{q^{n}-q}{q-1}>q^{n-1}
$$

as above. So $\left|G_{1}\right|_{p}<\mu_{1}\left(G_{1}\right)$, and $G_{1}$ has no Syl $p$-regular character. Then neither has $G$ by Lemma 2.5 .

\subsection{The groups $\mathrm{GU}_{n}(q), n>2$}

In this section, we consider the case where $p>2$ and a Sylow $p$-subgroup of $\mathrm{GU}_{n}(q)$ is abelian or abelian-by-cyclic. This implies $n<d p^{2}$, where $d$ is the order of $-q$ modulo $p$, equivalently, $d=2 e$ if $e$ is odd, $d=\frac{e}{2}$ if $e \equiv 2(\bmod 4)$, and $d=e$ if $4 \mid e$. So $d=1$ if and only if $e=2$, equivalently, $p \mid(q+1)$. Note that $e=2 e_{p}\left(q^{2}\right)$ if $e$ is even.

Lemma 10.5. Let $p$ be odd, and let $S$ be a Sylow $p$-subgroup of $G=\operatorname{GU}_{n}(q)$. Then $S$ is abelian and not cyclic if and only if $2 d \leq n<d p$.

Proof. If $X=\mathrm{GL}_{n}(q)$, then Sylow $p$-subgroups of $X$ are abelian if and only if $n<e p$. Let $S$ be a Sylow $p$-subgroup of $G$. We use Lemma 9.2. If $e \equiv 2(\bmod 4)$, then $|G|_{p}=\left|\operatorname{GL}_{n}\left(q^{2}\right)\right|_{p}$, so $S$ is abelian if and only if $n<e_{p}\left(q^{2}\right) p=\frac{e p}{2}=d p$. If $e$ is odd, then $|G|_{p}=\left|\mathrm{GL}_{\lfloor n / 2\rfloor}(q)\right|_{p}$, so $S$ is abelian if and only if $\left\lfloor\frac{n}{2}\right\rfloor<e p$, equivalently, $n<2 e p=d p$. If $4 \mid e$, then $|G|_{p}=\left|\mathrm{GL}_{\lfloor n / 2\rfloor}\left(q^{2}\right)\right|_{p}$, so $S$ is abelian if and only if $\left\lfloor\frac{n}{2}\right\rfloor<e_{p}\left(q^{2}\right) p$, equivalently, $n<e p=d p$. Similarly, $S$ is cyclic if and only if $n<2 d$. So the lemma follows. 
Lemma 10.6. Let $p>2, d>1$ and $\mathrm{SU}_{d p}(q) \leq G \leq \mathrm{GU}_{d p}(q)$. Then we have $|G|_{p}<\mu_{1}(G)$, and $G$ has no Syl $p_{p}$-regular character. This remains true if

$$
\mathrm{SU}_{n}(q) \leq G \leq \mathrm{GU}_{n}(q) \text { with } 2 d \leq n<d p .
$$

Proof. Note that $d>1$ means that $p$ does not divide $q+1$, so $|G|_{p}=\left|\operatorname{SU}_{d}(q)\right|_{p}$. So it suffices to prove the lemma for $G=\operatorname{SU}_{d p}(q)$. First assume that $d$ is odd, so $e \equiv 2(\bmod 4)$ and $d=\frac{e}{2}$. Then we have

$$
|G|_{p} \leq \frac{p\left(q^{e / 2}+1\right)^{p}}{(q+1)^{p}}=\frac{p\left(q^{d}+1\right)^{p}}{(q+1)^{p}} \quad \text { and } \quad \mu_{1}(G)=\frac{q^{d p}-q}{q+1} .
$$

In this case, $|G|_{p}<\mu_{1}(G)$. Similarly, if $e$ is odd, then $d=2 e$,

$$
|G|_{p} \leq \frac{p\left(q^{d}-1\right)^{p}}{(q+1)^{p}} \quad \text { and } \quad \mu_{1}(G)=\frac{q^{d p}-1}{q+1}
$$

so again $|G|_{p}<\mu_{1}(G)$.

Finally, assume that $4 \mid e$. Then $d=e$,

$$
|G|_{p} \leq \frac{p\left(q^{e}-1\right)^{p}}{\left(q^{2}-1\right)^{p}} \quad \text { and } \quad \mu_{1}(G)=\frac{q^{e p}-1}{q+1} .
$$

So $|G|_{p}<\mu_{1}(G)$ again. This implies that $G$ has no $\operatorname{Syl}_{p}$-regular character.

The proof of the additional statement is similar.

Thus, we are left with primes such that $p \mid(q+1)$. We first consider the case where $n<p$.

Lemma 10.7. Let $p \mid(q+1), 2<n<p$ and $\mathrm{SU}_{n}(q) \leq G \leq \mathrm{GU}_{n}(q)$. Then $G$ has no $\mathrm{Syl}_{p}$-regular character.

Proof. Let

$$
G_{1}=\left\{g \in G \mid \operatorname{det} g \text { is an element of } \mathrm{GU}_{1}(q) \text { of } p^{\prime} \text {-order }\right\} .
$$

Then $\left|G: G_{1}\right|=\left|Z_{p}\right|$, where $Z_{p}$ is the Sylow $p$-subgroup of $Z(G)$. It follows that $G=G_{1} \times Z_{p}$ as $Z_{p} \cap G_{1}=1$. By Lemma 2.6, the result for $G$ follows if we show that $G_{1}$ has no $\mathrm{Syl}_{p}$-regular character. In turn, this follows from the same result for $G^{\prime}=\operatorname{SU}_{n}(q)$ as $\left|G_{1}: G^{\prime}\right|$ is coprime to $p$. So we deal with $G^{\prime}$. Suppose the contrary, and let $\chi$ be a Syl $p$-regular character of $G^{\prime}$.

First, let $n=3$. Then $\chi(1)=\left|G^{\prime}\right|_{p}=(q+1)_{p}^{2}$ for $p>3$. By [20, Table V], $\mu_{1}\left(G^{\prime}\right)=q^{2}-q$. Let $q>4$. Then

$$
\chi(1)=\left|G^{\prime}\right|_{p}=(q+1)_{p}^{2}<2\left(q^{2}-q\right)=2 \mu_{1}\left(G^{\prime}\right) .
$$


So $\chi$ has a single non-trivial irreducible constituent $\rho$, and $\rho(1) \leq \chi(1)$. Again by [20, Table V], $q^{2}-q \leq \rho(1) \leq q^{2}-q+1$. As $\chi$ is $\operatorname{Syl}_{p}$-regular, $\left(\chi, 1_{G^{\prime}}\right) \leq 1$ (Lemma 2.1). Therefore, $\chi(1) \leq \rho(1)+1 \leq q^{2}-q+2$, which is false. The case with $q=4$ can be read off from the character table of $G^{\prime}$.

Let $n>3$, and let $V$ be the natural $\mathbb{F}_{q^{2}} G^{\prime}$-module. Let $b_{1}, \ldots, b_{n}$ be an orthogonal basis in $V$, and let $W=\left\langle b_{1}, b_{2}, b_{3}\right\rangle$. Then $W$ is a non-degenerate subspace of $V$ of dimension 3. Set $X=\left\{h \in G^{\prime} \mid h W=W, h b_{i} \in\left\langle b_{i}\right\rangle\right.$ for $\left.i=4, \ldots, n\right\}$ and $U:=O_{p}(X)$. Then $U \subseteq Z(X)$ and every element of $U$ acts diagonally on $W$. Let $X^{\prime}$ be the derived subgroup of $X^{\prime}$ and $P=X^{\prime} U$. Then $X^{\prime} \cong \mathrm{SU}_{3}(q)$ and $P / U \cong \mathrm{SU}_{3}(q)$ (as $p>3$ ). By the above, $\mathrm{SU}_{3}(q)$ has no $\mathrm{Syl}_{p}$-regular character. As $P$ contains a Sylow $p$-subgroup of $G^{\prime}$, the result follows from Lemma 2.5.

Lemma 10.8. Let $\mathrm{SU}_{3}(8) \leq G \leq \mathrm{GU}_{3}(8)$. Then $G$ has no $\mathrm{Syl}_{3}$-regular character.

Proof. By Lemma 2.5, it suffices to prove that $\mathrm{PSU}_{3}(8)$ and $\mathrm{PGU}_{3}(8)$ have no $\mathrm{Syl}_{3}$-regular character. Suppose the contrary, and let $\chi$ be a Syl ${ }_{3}$-regular character of any of these groups. As $\left|\mathrm{PGU}_{3}(8)\right|_{3}=243$, we have $\chi(1) \leq 243$. Let $\rho$ be an irreducible constituent of $\chi$. Then $\rho(1) \leq 243$. By [1], $\rho(1) \in\{1,56,57,133\}$, and the characters of degree $1,56,133$ are positive at the class 9A, whereas those of degree 57 vanish at this class. It follows that $\rho(1)=57$, but then $\rho$ is positive at the class $3 \mathrm{C}$. This is a contradiction.

Lemma 10.9. Let $H=\mathrm{SU}_{p}(q)$, where $p>2, p \mid(q+1)$, or $H=\mathrm{SL}_{p}(q)$, where $p>2, p \mid(q-1)$, and $h=\operatorname{diag}\left(1, \varepsilon, \varepsilon^{2}, \ldots, \varepsilon^{p-1}\right) \in H$ with $\varepsilon \in \mathbb{F}_{q^{2}}^{\times}$a primitive $p$-th root of unity. Let $\chi$ be an irreducible character of $H$ whose kernel has order prime to $p$. Then $\chi(h)=0$.

Proof. The element $h$ is written in an orthogonal basis of the underlying vector space in the unitary case. Then $h \in E$, where $E \leq H$ is an extraspecial group of order $p^{3}$ such that $Z(E)=Z(H)$. The restriction of $\chi$ to $E$ is a direct sum of irreducible representations of $E$ non-trivial on $Z(E)$. It is well known and can be easily checked that the character of every such representation vanishes at $h$. So the claim follows.

Let $H=\mathrm{GU}_{n}(q)$ or $\mathrm{GL}_{n}(q)$ with $n>2$. Weil representations of these groups were studied by Howe [7] and other authors, and have many applications, mainly because their irreducible constituents (which we call irreducible Weil representations) essentially exhaust the irreducible representations of degree $\mu_{1}(H)$ and $\mu_{2}(H)$. More details are given below for $n=p, p$ odd. Let $M$ be the underlying space of the Weil representation of $H$. Then $M=\oplus_{\zeta \in \operatorname{Irr}(Z(H))} M_{\zeta}$, where $M_{\zeta}=\{m \in M \mid z m=\zeta(z) m$ for $z \in Z(H)\}$. In general, $H$ is irreducible on $M_{\zeta}$, 
except for the case where $H=\operatorname{GL}_{p}(q)$ and $\zeta=1_{Z(H)}$. In this case, $M_{\zeta}$ is a sum of a one-dimensional and an irreducible $H$-invariant subspace.

So the irreducible Weil representations $\rho$ of $H$ of dimension greater than 1 are parameterised by their restriction to $Z(H)$, and each of them remains irreducible under restriction to $H^{\prime}=\mathrm{SU}_{n}(q)$ or $\mathrm{SL}_{n}(q)$. By [20], every irreducible representation of $H^{\prime}$ of degree $\mu_{1}(H)$ and $\mu_{2}(H)$ is an irreducible Weil representation. Moreover, every irreducible representation of $H$ of degree $\mu_{1}(H)$ and $\mu_{2}(H)$ is obtained from an irreducible Weil representation by tensoring with a one-dimensional representation.

Lemma 10.10. Let $p>2$ and $H=\mathrm{GU}_{p}(q)$, where $p \mid(q+1),(p, q) \neq(3,2)$, or $\mathrm{GL}_{p}(q)$, where $p \mid(q-1)$. Let $\zeta \in \operatorname{Irr}(Z(H))$. Let $\rho=\rho_{\zeta}$ be the character of an irreducible constituent of the Weil representation $\omega$ of $H$ labelled by $\zeta$ (where $\rho(1)>1)$. Let $h$ be as in Lemma 10.9. Then $\rho(h) \in\{0, p, p-1\}$, except for the case with $G=\mathrm{GL}_{p}(q)$ and $\zeta=1_{Z(H)}$, where $\rho(h)=p-2$. In addition, $\rho(h) \neq 0$ if and only if $\rho(z)=1$ for an element $z \in Z(H)$ of order $p$.

Proof. We only consider the case $H=\mathrm{GU}_{p}(q)$ as the case $H=\mathrm{GL}_{p}(q)$ is similar.

We have $\rho(z)=\rho(1) \zeta(z)$. Set $Z=Z(H)$. Let $X=\langle Z, h\rangle$. Let $\varepsilon_{i}$ be the character of $\langle h\rangle$ such that $\varepsilon_{i}(h)=v^{i}$, where $v$ is a fixed $p$ th root of unity, $i=1, \ldots, p$. Then the multiplicity of the eigenvalue $v^{i}$ of $\rho(h)$ equals $\left(\left.\omega\right|_{X}, \zeta \cdot \varepsilon_{i}\right)$.

Recall that $\omega(x)=-(-q)^{d}$, where $d$ is the multiplicity of the eigenvalue 1 of $x$ as a matrix in $\mathrm{GU}_{p}(q)$. Therefore, $\omega(1)=q^{n}$, and if $x=z h^{k}$ and $z^{p} \neq 1$, then $\omega(x)=-1$; if $z^{p}=1, h \neq 1$, then $\omega(x)=q$ (also for $z=1$ ).

We compute $|X| \cdot\left(\left.\omega\right|_{X}, \zeta \cdot \varepsilon_{i}\right)=\sum_{x \in X} \omega(x) \zeta(z) \varepsilon_{i}(h)$, where $x=z h$. Note that $\omega(x)$ is an integer, so $\omega$ is self-dual. Let $Z_{p}$ be the subgroup of order $p$ in $Z$, and $X_{p}=\left\langle Z_{p}, h\right\rangle$. Then

$$
\sum_{x \in X} \omega(x) \zeta(z) \varepsilon_{i}(h)=\sum_{x \in X_{p}} \omega(x) \zeta(z) \varepsilon_{i}(h)+\sum_{x \notin X_{p}} \omega(x) \zeta(z) \varepsilon_{i}(h) .
$$

We first show that the second sum equals 0 if $i<p$. Note that $x=z h \notin X_{p}$ is equivalent to $z \notin Z_{p}$. Therefore, $d=d(x)=0$ for $x \notin X_{p}$, and $\omega(x)=-1$. For $z$ fixed, we have a partial sum $\zeta(z) \sum_{h} \varepsilon_{i}(h)$, and $\sum_{h} \varepsilon_{i}(h)=0$, as claimed.

If $i=p$ and $\zeta \neq 1_{Z}$, then

$$
\begin{aligned}
\sum_{x \notin X_{p}} \omega(x) \zeta(z) \varepsilon_{i}(h) & =-p \sum_{z \notin Z_{p}} \zeta(z) \\
& =-p\left(\sum_{z \in Z} \zeta(z)-\sum_{z \in Z_{p}} \zeta(z)\right)=p^{2}
\end{aligned}
$$


as $\zeta(z)=1$ for $z \in Z_{p}$. If $i=p$ and $\zeta=1_{Z}$, then

$$
\sum_{x \notin X_{p}} \omega(x) \zeta(z) \varepsilon_{i}(h)=-\left(|X|-\left|X_{p}\right|\right)=-p(q+1)+p^{2} .
$$

Next, we compute $\sum_{x \in X_{p}} \omega(x) \zeta(z) \varepsilon_{i}(h)$. Observe that $\zeta(z)=1$ for $z \in Z_{p}$, so this sum simplifies to $\sum_{z h \in X_{p}} \omega(z h) \varepsilon_{i}(h)$. Note that $d(z h)=1$ if $h \neq 1$ and any $z \in Z_{p}$. So if $h \neq 1$, then $\omega(z h)=q$. If $h=1$, then $d(z h)=d(z)=0$ for $z \neq 1$, so $\omega(z)=-1$, and $\omega(1)=q^{p}$.

Therefore, we have

$$
\begin{aligned}
\sum_{z h \in X_{p}} \omega(z h) \varepsilon_{i}(h) & =\sum_{z \in Z_{p}, h \neq 1} \omega(z h) \varepsilon_{i}(h)+\sum_{z \in Z_{p}, z \neq 1} \omega(z)+q^{p} \\
& =\sum_{z \in Z_{p}, h \neq 1} q \cdot \varepsilon_{i}(h)+\sum_{z \in Z_{p}, z \neq 1}(-1)+q^{p} \\
& =p q \sum_{h \neq 1} \varepsilon_{i}(h)-(p-1)+q^{p} .
\end{aligned}
$$

(i) Let $i \neq p$. Then $\sum_{h \neq 1} \varepsilon_{i}(h)=-1$, and the last sum equals

$$
-p q-p+1+q^{p}=q^{p}+1-p(q+1) .
$$

(ii) Let $i=p$. Then $\sum_{h \neq 1} \varepsilon_{i}(h)=p-1$, and the last sum equals

$$
p q(p-1)-(p-1)+q^{p}=q^{p}+1+p^{2} q-p q-p .
$$

Therefore,

$$
|X| \cdot\left(\left.\omega\right|_{X}, \zeta \cdot \varepsilon_{i}\right)= \begin{cases}q^{p}+1-p(q+1) & \text { if } i \neq p, \\ q^{p}+1+(p-1) p(q+1) & \text { if } i=p, \zeta \neq 1_{Z}, \\ q^{p}+1+(p-2) p(q+1) & \text { if } i=p, \zeta=1_{Z} .\end{cases}
$$

In particular, the multiplicities of eigenvalue $v^{i}$ for $i \neq p$ of $h$ on the module $M_{\zeta}$ for fixed $\zeta$ are the same. As $\sum_{i \neq p} v^{i}=-1$, the trace of $h$ on $M_{\zeta}$ for $\zeta \neq 1_{Z}$ with $\zeta\left(Z_{p}\right)=1$ equals

$$
(1 /|X|)\left(q^{p}+1+(p-1) p(q+1)-\left(q^{p}+1-p(q+1)\right)=p\right.
$$

as $|X|=p(q+1)$. Similarly, if $\zeta=1_{Z}$, then the trace in question equals $p-1$. In other words, if $\omega_{\zeta}$ is the character of $M_{\zeta}$ and $\zeta\left(Z_{p}\right)=1$, then $\omega_{\zeta}(h)=p$ for $\zeta \neq 1_{Z}$ and $p-1$ otherwise. 
Lemma 10.11. Let $H=\mathrm{GU}_{p}(q)$, where $p \mid(q+1), p>2,(p, q) \neq(3,2)$, or $\mathrm{GL}_{p}(q)$, where $p \mid(q-1)$. Then $H$ has no $\mathrm{Syl}_{p}$-regular character. The same is true for $H^{\prime}=\mathrm{SU}_{p}(q)$ and $\mathrm{SL}_{p}(q)$ and for all groups $X$ with $H^{\prime} \leq X \leq H$.

Proof. Set $G=H / O_{p}(Z(H))$. By Lemma 2.5, it suffices to prove the lemma for $G$ in place of $H$.

Suppose the contrary. Let $\chi$ be a Syl $p$-regular character of $G$, and let $\lambda$ be an irreducible constituent of $\chi$. We first observe that $\lambda(1)<\mu_{3}(G)$, and hence, by [20], $\lambda(1) \in\left\{1, \mu_{1}(G), \mu_{2}(G)\right\}$.

Indeed, note that

$$
|G|_{p}= \begin{cases}p|q+1|_{p}^{p-1} & \text { in the unitary case } \\ p|q-1|_{p}^{p-1} & \text { in the linear case. }\end{cases}
$$

By [20, Table IV],

$$
\mu_{3}(G) \geq \mu_{3}\left(H^{\prime}\right) \geq \frac{\left(q^{p}+1\right)\left(q^{p-1}-q^{2}\right)}{(q+1)\left(q^{2}-1\right)},
$$

respectively,

$$
\frac{\left(q^{p}-1\right)\left(q^{p-1}-q^{2}\right)}{(q-1)\left(q^{2}-1\right)} \text { if } p>3 .
$$

This value is greater than $|G|_{p}$. Let $p=3$. Then

$$
\mu_{3}(G) \geq \mu_{3}\left(H^{\prime}\right) \geq \frac{\left(q^{2}-q+1\right)(q-1)}{3},
$$

respectively, $\frac{\left(q^{2}-1\right)(q-1)}{3}$ for $q>4$. Again, $|G|_{3}<\mu_{3}(G)$ unless $G=\operatorname{PGU}_{3}(8)$ or $\mathrm{PGL}_{3}(4)$. The former case is settled in Lemma 10.8 .

Let $G=\mathrm{PGL}_{3}(4)$. Then $|G|_{3}=27$. In this case, $\mu_{1}(G)=20, \mu_{2}(G)=35$ and $\mu_{3}(G)=45$. So $\lambda(1) \leq|G|_{p}$ implies $\lambda(1) \leq 20$. The character of degree 20 is positive at class $3 \mathrm{~A}$, a contradiction.

So $|G|_{p} \leq \mu_{2}(G)$. As mentioned prior to Lemma 10.10, $\lambda$ is either one-dimensional or can be seen as a character of $H$ obtained from an irreducible Weil character by tensoring with a linear character of $H$. Let $h \in H$ as in Lemma 10.10. Then $h \in H^{\prime}$, so tensoring can be ignored, and we can assume that $\lambda$ is an irreducible Weil character of $H$. Then, by Lemma $10.10, \lambda(h) \in\{0, p, p-1\}$ in the unitary case and $\lambda(h) \in\{0, p, p-2\}$ in the linear case. If $\lambda(1)=1$, then $\lambda(h)=1$. So $\lambda(h) \geq 0$. As $\chi$ is $p$-vanishing and $|h|=p$, we have $\chi(h)=0$. So $\lambda(h)=0$ for every irreducible constituent of $\chi$. This is false as $\lambda$ is trivial on $O_{p}(Z(H))$ by the definition of $G$, and hence $\lambda(h) \neq 0$ by Lemma 10.10 . This is a contradiction. As irreducible Weil representations of $H$ remain irreducible upon restriction to $H^{\prime}$, this argument works for intermediate groups $X$ too. 
Lemma 10.12. Let $p>2$, and let $G$ be a group such that $\operatorname{SL}_{n}(q) \leq G \leq \mathrm{GL}_{n}(q)$ with $2 e<n \leq e p$, or $\mathrm{SU}_{n}(q) \leq G \leq \mathrm{GU}_{n}(q)$ with $2 d<n \leq d p,(n, q) \neq(3,2)$. Then $G$ and $G / O_{p}(G)$ have no $\mathrm{Syl}_{p}$-regular character.

Proof. For the unitary case with $d>1$, the result for $G$ is stated in Lemma 10.6. The case with $d=1$ and $n<p$ is dealt with in Lemma 10.7, and the remaining case $d=1$ and $n=p$ is examined in Lemma 10.11.

Let $H=\mathrm{GL}_{n}(q)$. The result for $e>1, q>2$ follows from Lemma 10.1, and that for $e>1, q=2$ is proved in Lemma 10.3. The result for $e=1, n=p$ is stated in Lemma 10.11. The case with $e=1, n<p$ is examined in Lemma 10.4.

The statement on $G / O_{p}(G)$ follows from Lemma 2.5.

Lemma 10.13. For $p>2$, let $H=\mathrm{GL}_{n}(q), H^{\prime}=\mathrm{SL}_{n}(q)$ with ep $<n<e p^{2}$, or $H=\mathrm{GU}_{n}(q), H^{\prime}=\mathrm{SU}_{n}(q)$ with $d p<n<d p^{2}$. Let $G$ be a group such that $H^{\prime} \leq G \leq H$. Then $G$ has no Syl ${ }_{p}$-regular character unless $p=3, H=\mathrm{GU}_{4}(2)$.

Proof. Suppose the contrary, and let $\chi$ be a Syl $p$-regular character of $G$.

Suppose first that $e>1, d>1$. Note that $G$ has a subgroup $X$, say, isomorphic to, $\mathrm{SL}_{e p}(q) \times \mathrm{SL}_{n-e p}(q)$, respectively, $\mathrm{SU}_{d p}(q) \times \mathrm{SU}_{n-d p}(q)$, and $|G: X|_{p}=1$. Let $S$ be a Sylow $p$-subgroup of $\mathrm{SL}_{n-e p}(q)$, respectively, $\operatorname{SU}_{n-d p}(q)$, and let $Y=S \times \mathrm{SL}_{e p}(q)$, respectively, $S \times \mathrm{SU}_{d p}(q)$. As $|G: Y|_{p}=1$, by Lemma 2.5, $r_{Y / S}^{G}(\chi)$ is a $\operatorname{Syl}_{p}$-regular character of $Y / S \cong \mathrm{SL}_{e p}(q)$, respectively, $\operatorname{SU}_{d p}(q)$. This contradicts Lemma 10.12 unless, possibly, if $G=\mathrm{SU}_{n}(2)$ and $p=3$. As $d>1$, this case does not occur.

Next, suppose that $e=d=1$, that is, $p \mid(q-1)$ or $q+1$. Then we refine the above argument. Set $D=\mathrm{GL}_{p}(q)$ or $\mathrm{GU}_{p}(q)$. Then $Y / S \cong D$. Set $Y_{1}=G \cap Y$. Then $Y_{1}$ is normal in $Y$, and hence $O_{p}\left(Y_{1}\right)=Y_{1} \cap O_{p}(Y)$. As $Y / S \cong D$, it follows that $Y / O_{p}(Y)=D / O_{p}(D)=D / O_{p}(Z(D))$, and hence $E:=Y_{1} / O_{p}\left(Y_{1}\right)$ is a non-central normal subgroup of $D / O_{p}(Z(D))$. By Lemma 2.5, $r_{Y_{1} / O_{p}\left(Y_{1}\right)}^{G}(\chi)$ is a $S_{1}$-regular character of $E=Y_{1} / O_{p}\left(Y_{1}\right)$. However, by Lemma $10.12, E$ has no Syl $p$-regular character unless $D=\mathrm{GU}_{3}(2)$ and $p=3$. So we are left with the case $H=\mathrm{GU}_{n}(2), p=3$ and $3<n<9$.

The group $H=\mathrm{GU}_{4}(2)$ is excluded by assumption, and so we first consider $H=\mathrm{GU}_{5}(2)$. As $H=H^{\prime} \times Z(H)$, it suffices to deal with $G=\mathrm{SU}_{5}(2)$. Suppose the contrary, and let $\chi$ be a $\operatorname{Syl}_{3}$-regular character of $G$. Then we have $\chi(1)=|G|_{p}=243$. Let $\lambda$ be an irreducible constituent of $\chi$, so $\lambda(1) \leq 243$. If $g \in G$ is an element from class $3 \mathrm{E}$, then $\lambda(g)>0$ unless $\lambda(1)=176$ or 220 . As $\chi(g)=0$, there is a constituent $\lambda_{1}$, say, of $\chi$ such that $\lambda_{1}(1) \in\{176,220\}$. Then $\lambda(1) \leq 67=243-176$. Pick $h \in G$ from the class 3 F. Then $\lambda_{1}(h)>0$, and if $\lambda(1) \leq 67$, then $\lambda(h)>0$ unless $\lambda(1)=10$. So $\chi$ must have a constituent $\lambda_{2}$, 
say, of degree 10. Then $\lambda_{2}(g)=4$. If $\lambda_{1}(1)=176$ then $\lambda_{1}(g)=-4$, and hence $\left(\chi-\lambda_{1}-\lambda_{2}\right)(g)=0$. As $\lambda(g)>0$ if $\lambda(1) \leq 67$, it follows that $\chi=\lambda_{1}+\lambda_{2}$, but then $0=\chi(h)=\lambda_{1}(h)+\lambda_{2}(h)=3$, a contradiction. So $\lambda_{1}(1)=220$, and the other constituents are of degree at most 23. As $\lambda_{1}(g)=-5, \lambda_{2}(g)=4$, we have $\left(\chi-\lambda_{1}-\lambda_{2}\right)(g)=-1$; in particular, for the other constituents $\lambda$ of $\chi$, we have $\lambda(g) \leq 1$. By [1], this implies $\lambda(1)=1$, and $\lambda$ must occur with multiplicity 1 , whence $\chi(1)=220+10+1=231$, a contradiction.

Let $H=\mathrm{GU}_{6}(2)$. By Lemma 2.5 , it suffices to deal with $X:=\mathrm{PGU}_{6}(2)$. Set $X^{\prime}=\operatorname{PSU}_{6}(2)$. Then $\left|X^{\prime}\right|_{3}=3^{6}=729$, and the irreducible characters of $X^{\prime}$ of degree less than 616 are positive on class 3A. In addition, $|X|_{3}=3^{7}=2187$, and the irreducible characters of $X$ of degree less than 2187 and not equal to 616 are positive on class 3A. Let $\chi$ be a Syl ${ }_{3}$-regular character of $X$ or $X^{\prime}$. Then the irreducible character $\mu$ of degree 616 is a constituent of $\chi$. Note that $\tau(3 \mathrm{~A})=-14$. If $\chi \in \operatorname{Irr}\left(X^{\prime}\right)$, then the sum of the other constituents of $\chi$ is at most 113. By [1], they are of degree 1 or 22 . The trivial character cannot occur with multiplicity greater than 1 , so 113 or 112 must be a multiple of 22 , which is false. Let $\chi \in \operatorname{Irr}(X)$. Note that the multiplicity of $\mu$ in $\chi$ is at most 3, and if $\mu$ occurs with multiplicity 3 , then the sum of the other constituents of $\chi$ is at most $2187-1848=339$. The irreducible characters of degree at most 339 have degrees 252, 232, 22, 1 , and all of them as well as $\mu$ are positive at class 3C. This is a contradiction. Suppose that $\mu$ occurs once. Then the sum of the other constituent values at class $3 \mathrm{~A}$ is -14 . It follows that these constituents may only be of degrees 770, 252, 232, 22 , 1. Inspecting [1], one observes that all of them as well as $\mu$ are positive at class $3 \mathrm{C}$. This is a contradiction. So the multiplicity of $\mu$ must be 2 . Then the sum of the other constituent values at class $3 \mathrm{~A}$ is -28 . Therefore, the degrees of the other constituents may only be $770,560,385,252,232,22,1$. Let $v$ be the character of degree 385 . Then $v(3 \mathrm{~A})=25$. If $(\chi, v)>0$, then the sum of the other constituent values at class $3 \mathrm{~A}$ is -3 . The trivial character is the only one whose value is at most 3 . As this cannot occur twice, we get a contradiction. Therefore, $(\chi, v)=0$. As above, this contradicts $\chi(3 C)=0$. This completes the analysis of the case with $n=6$.

Let $n=7$. Then $H^{\prime}=\mathrm{SU}_{7}(2)$ contains a subgroup isomorphic to $\mathrm{GU}_{6}(2)$, which contains a Sylow 3 -subgroup of $H^{\prime}$. So the result for this case follows from $n=6$. In addition, $H=H^{\prime} \cdot Z(H)$, so we are done by Lemma 2.5 .

Similarly, the result for $n=8$ follows from that with $n=7$.

Remark 10.14. The group $\mathrm{SU}_{4}(2)$ has an irreducible projective character of degree 81 (for $p=3$ ), and hence $H=\mathrm{GU}_{4}(2)=\mathrm{SU}_{4}(2) \times Z(H)$ has a projective character of degree $|H|_{3}=243$. 
Theorem 10.15. Let $p>2$, and let $G$ be a group such that $\operatorname{SL}_{n}(q) \leq G \leq \operatorname{GL}_{n}(q)$ or $\mathrm{SU}_{n}(q) \leq G \leq \mathrm{GU}_{n}(q)$. Suppose that Sylow p-subgroups of $G / Z(G)$ are not cyclic. Then $G$ has no Steinberg-like character unless $p=3$ and

$$
G \in\left\{\mathrm{SU}_{3}(2), \mathrm{GU}_{3}(2), \mathrm{SU}_{4}(2), \mathrm{GU}_{4}(2)\right\} .
$$

Proof. If $n \geq e p^{2}$ in the linear case and $n \geq d p^{2}$ in the unitary case, then the result follows from Proposition 9.6 for $G / O_{p}(G)$ in place of $G$, and then for $G$ in view of Lemma 2.5.

- If $e p<n<e p^{2}$ in the linear case and $d p<n<d p^{2}$ in the unitary case, then the result follows from Lemma 10.13.

- If $2 e \leq n \leq e p$ in the linear case and $2 d \leq n \leq d p$ in the unitary case, then the result follows from Lemma 10.12.

- If $n<2 e$ in the linear case and $n<2 d$ in the unitary case, then Sylow $p$-subgroups of $G / Z(G)$ are cyclic.

Remark 10.16. Proposition 9.6 gives a better bound for $n$, but this does not yield an essential advantage as the cases with $n=e(p+1)$ and $d(p+1)$ are not covered by Proposition 9.6, and we have to use Lemma 10.13 anyway.

\subsection{The symplectic and orthogonal groups for $p>2$}

\section{Lemma 10.17. Let}

$$
G= \begin{cases}\operatorname{Sp}_{2 n}(q), & q \text { even, } n \geq 2,(n, q) \neq(2,2),(3,2), \\ \operatorname{Spin}_{2 n+1}(q), & q \text { odd }, n \geq 3,(n, q) \neq(3,3), \\ \operatorname{Spin}_{2 n}^{ \pm}(q), & n \geq 4\end{cases}
$$

Suppose that Sylow p-subgroups of $G$ are abelian. Then $|G|_{p}<\mu_{1}(G)$.

Proof. Let $S \in \operatorname{Syl}_{p}(G)$. As $S$ is abelian, we have $p>2$ and $|S| \leq(q+1)^{n}$. If $G=\operatorname{Sp}_{2 n}(q)$, where $q$ is even, $n \geq 2$ and $(n, q) \neq(2,2)$, or $\operatorname{Spin}_{2 n+1}(3)$, then (see [20, Table II])

$$
\mu_{1}(G) \geq \frac{\left(q^{n}-1\right)\left(q^{n}-q\right)}{2(q+1)} .
$$

This is greater than $(q+1)^{n} \geq|S|$, except for the cases where $q=2$ and $n \leq 6$. For these cases, the statement follows by inspection. If $G=\operatorname{Spin}_{2 n+1}(q)$, where $q>3$ is odd and $n \geq 3$, then $\mu_{1}(G) \geq \frac{q^{2 n}-1}{q^{2}-1}$. Again, $\mu_{1}(G)>(q+1)^{n}$, whence the result. The cases with $G=\operatorname{Spin}_{2 n}^{ \pm}(q)$ with $n \geq 4$ are similar; see [20, Theorem 7.6]. 
Proposition 10.18. Let $e$ be odd and $p>2$, and let $H=\operatorname{Sp}_{2 n}(q)$ with $n>1$, $\mathrm{GO}_{2 n+1}(q)$ with $n>2, \mathrm{GO}_{2 n}^{+}(q)$ with $n>3$, or $\mathrm{GO}_{2 n+2}^{-}(q)$ with $n>2$. Suppose that $2 e \leq n<e p^{2}$. Then $H$ has no $\mathrm{Syl}_{p}$-regular character.

Proof. Let $S \in \operatorname{Syl}_{p}(H)$. By Lemma 9.2(1), $S$ is conjugate to a Sylow $p$-subgroup of a subgroup $H_{1} \cong \mathrm{GL}_{n}(q)$ of $H$. By Lemmas 10.1, 10.3, 10.12 and 10.13, $\mathrm{GL}_{n}(q)$ for $2 e \leq n<e p^{2}$ has no Syl $p$-regular character unless, possibly, when $n=2$.

Let $n=2$, so $H=\operatorname{Sp}_{4}(q), e=1$ and $p \mid(q-1)$. Then $|H|_{p}=|q-1|_{p}^{2}$. If $q$ is even, then

$$
\mu_{1}(G)=\frac{q(q-1)^{2}}{2} \text { for } q>2 .
$$

This is greater than $|H|_{p}$, whence the result. If $q$ is odd, then $|H|_{p} \leq \frac{(q-1)^{2}}{4}$ and $\mu_{1}(H)=\frac{q^{2}-1}{2}$. So, again, $|H|_{p}<\mu_{1}(H)$.

Proposition 10.19. Let e be even, $p>2$, and let $H=\operatorname{Sp}_{2 n}(q)$ with $n>1$ and $(n, q) \neq(2,2), \mathrm{GO}_{2 n+1}(q)$ with $q$ odd and $n>2$, or $\mathrm{GO}_{2 n}^{ \pm}(q)$ with $n>3$. Suppose that $2 e \leq 2 n<e p^{2}$. Then $H$ has no $\mathrm{Syl}_{p}$-regular character.

Proof. Write $2 n=e k+m$ with $m<e$, where $k>1$ is an integer. As $H$ contains a subgroup $H_{1}$ with $\left(\left|H: H_{1}\right|, p\right)=1, H_{1} \cong \mathrm{Sp}_{k e}(q)$ or $\mathrm{GO}_{k e+1}(q)$, respectively, it suffices to prove the lemma for $2 n=k e$. Let $2 n=k e$. By Lemma 9.4, a Sylow $p$-subgroup of $H$ is contained in a subgroup isomorphic to $\operatorname{GU}_{k}\left(q^{e / 2}\right)$. By Lemma 10.12 for $2<k \leq p$ and Lemma 10.13 for $p<k<p^{2}$ (with $d=1$ and $q^{e / 2}$ in place of $\left.q\right)$, the group $\mathrm{GU}_{k}\left(q^{e / 2}\right)$ with $\left(k, q^{e / 2}\right) \neq(3,2)$ has no $\mathrm{Syl}_{p^{-}}$ regular character, whence the claim. (The exceptional case $H=\operatorname{Sp}_{6}(2), p=3$ is considered below.)

Let $k=2$. Then $H=\operatorname{Sp}_{2 e}(q)$ and $p \mid\left(q^{e / 2}+1\right)$. Then $|H|_{p}=\left|q^{e / 2}+1\right|_{p}^{2}$. If $q$ is even, then

$$
\mu_{1}(G)=\frac{\left(q^{e}-1\right)\left(q^{e}-q\right)}{2(q+1)} \text { for } q>2 .
$$

This is greater than $|H|_{p}$, whence the result. If $q$ is odd, then

$$
|H|_{p} \leq \frac{\left(q^{e / 2}+1\right)^{2}}{4} \text { and } \quad \mu_{1}(H)=\frac{q^{e}-1}{2} .
$$

So $|H|_{p}<\mu_{1}(H)$, whence the result.

A similar argument works if $H=\mathrm{GO}_{k e+1}(q)$ as well as for $H=\mathrm{GO}_{k e}^{-}(q)$ with $k$ odd, and for $H=\mathrm{GO}_{k e}^{+}(q)$ with $k>2$ even, except when $H=\mathrm{GO}_{8}^{+}(2)$ and $e=2$. 
Let $H=\mathrm{GO}_{8}^{+}$(2) and $e=2$, so $p=3$. Then $|G|_{3}=243$, and the irreducible characters of degree less than 243 are of degrees 1, 28, 35, 50, 84, 175, 210. Let $\chi$

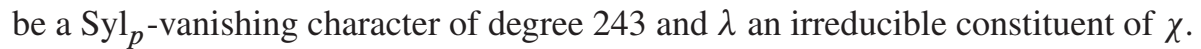
By [1], $\lambda(3 E)>0$ whenever $\lambda(1) \leq 243$. This is a contradiction as $\chi(3 E)=0$.

Let $k=2$ and $H=\mathrm{GO}_{2 e}^{+}(q)$. Then

$$
|H|_{p}=\left|q^{e / 2}+1\right|_{p}^{2}<\mu_{1}(H)=\frac{\left(q^{e}-1\right)\left(q^{e-1}-1\right)}{q^{2}-1}
$$

for $q>2$ and $q=2, e>4$. (If $q=2, e=4$, then $p=5$, and it follows that $|H|_{5}=25<\mu_{1}(H)=28$.) So the result follows. (The case $e=2$ has been examined above.)

Suppose that $H=\mathrm{GO}_{2 n}^{-}(q)$ with $k$ even or $H=\mathrm{GO}_{2 n}^{+}(q)$ with $k$ odd. Then some Sylow $p$-subgroup of $H$ is contained in a subgroup $H_{1}$ isomorphic to, respectively, $\mathrm{GO}_{2 n-e}^{-}(q)$ or $\mathrm{GO}_{2 n-e}^{+}(q)$. Note that $2 n-e=(k-1) e$. For the groups $H_{1}$, the result has been proven above, except for the cases where $k-1=1$ or $(k-1) e \leq 6$. However, if $k-1=1$, then Sylow $p$-subgroups of $H_{1}$, and hence of $H$, are cyclic, and this case has been examined in Propositions 3.1 and 4.4. Let $(k-1) e \leq 6$. As $k-1>1$, we have $k=3, e=2$ as $e$ is even. Then $H=\mathrm{GO}_{8}^{-}(q)$ and $\mu_{1}(H)=q\left(q^{4}+1\right)$ (see [20]). If $p>3$, then $|H|_{p}=|q+1|_{p}^{3}$; otherwise, $|H|_{3}=3|q+1|_{3}^{3}$. Then $|H|_{p}<\mu_{1}(H)$ unless $q=2$.

Let $q=2, p=3$. Then $|G|_{3}=81$. By [1], the irreducible characters of degree less than 81 are of degrees $1,26,52$. Therefore, only these characters can occur as irreducible constituents of a $\mathrm{Syl}_{3}$-regular character $\chi$. However, the values of these characters at an element $g \in G$ in class $9 \mathrm{~A}$ are $1,2,1$, in particular, positive. As $\chi(g)=0$, this is a contradiction.

Suppose that $G=\operatorname{Sp}_{6}(2)$ and $p=3$. Then $|S|=81$. Let $\tau$ be an irreducible constituent of $\chi$. Then $\tau(1) \leq 81$. Let $g \in G$ belong to the conjugacy class $3 \mathrm{C}$ in the notation of [1]. By inspection of the character table of $G$, one observes that $\tau(g) \geq 0$ whenever $\tau(1) \leq 81$. Therefore, $\tau(g)=0$ for every irreducible constituent $\tau$ of $\chi$. This implies $\tau(1) \in\{21,27\}$; see [1]. However, such a character takes positive values at the elements of class $3 \mathrm{~A}$. So this case is ruled out.

Remark 10.20. Let $G$ be the universal covering group of $\mathrm{SO}_{8}^{+}(2)$. One observes that $G$ has a $\mathrm{Syl}_{5}$-regular character and no $\mathrm{Syl}_{3}$-regular characters. If $H=\mathrm{Sp}_{4}(2)$, then $H$ has Steinberg-like characters for $p=3$, both reducible and irreducible.

\section{Classical groups at $p=2$}

In this section, we investigate $\mathrm{Syl}_{p}$-regular and Steinberg-like characters of simple classical groups over fields of odd order $q>3$ at the prime $p=2$. 


\subsection{Linear and unitary groups at $p=2$}

We first deal with the smallest case.

Proposition 11.1. Let $q>3$ be odd.

(a) Let $G=\operatorname{PSL}_{2}(q)$. Then $G$ has a reducible $\mathrm{Syl}_{2}$-regular character if and only if $q+1=2^{k}$ for some $k \geq 3$ or if $q=5$.

(b) Let $G=\mathrm{SL}_{2}(q)$. Then $G$ has a reducible $\mathrm{Syl}_{2}$-regular character if and only if $q \pm 1$ is a 2-power.

Proof. (a) The 2-part of $|G|$ is $|q-1|_{2}$ if $q \equiv 1(\bmod 4)$ and $|q+1|_{2}$ else. The smallest non-trivial character degree is $\frac{q+1}{2}$ in the first case, $\frac{q-1}{2}$ in the second. It follows that there cannot be $\mathrm{Syl}_{2}$-regular characters in the first case unless $q=5$. In the second case, it follows from the character table of $G$ that the sum of the trivial and the Steinberg character is $\mathrm{Syl}_{2}$-regular when $q+1$ is a power of 2, and there are no cases otherwise. If $q=5$, then there are two reducible $\mathrm{Syl}_{2}$-regular characters of degree 4 by [1].

(b) Let $\chi$ be a reducible $\operatorname{Syl}_{2}$-regular character. Let $1 \neq z \in Z(G)$. Then we have $\chi=\chi_{1}+\chi_{2}$, where $\chi_{1}(z)=\chi_{1}(1)$ and $\chi_{2}(z)=-\chi_{2}(1)$. By Lemma 2.5 (with $P=G, U=Z(G)$ ), $\chi_{1}$ is a $\operatorname{Syl}_{2}$-regular character for $G / Z(G)=\operatorname{PSL}_{2}(q)$. If $\chi_{1}$ is irreducible, then $q \pm 1$ is a 2-power by Proposition 3.1 (2); by (a), this is also true if $\chi_{1}$ is reducible. So $q \pm 1$ is a 2-power.

Then there are irreducible characters $\chi_{1}, \chi_{2}$ such that $\chi_{1}+\chi_{2}$ is 2-vanishing of degree $2(q \pm 1)=|G|_{2}$. Indeed, using the character table of $G$, one observes that there exist irreducible characters $\chi_{1}, \chi_{2}$ of $G$ that vanish at non-central 2-elements of $G$, and such that $\chi_{1}(z)=\chi_{1}(1)$ and $\chi_{2}(z)=-\chi_{2}(1)$. It follows that $\chi_{1}+\chi_{2}$ is a reducible $\mathrm{Syl}_{2}$-regular character.

Recall that $\mu_{3}(G)$ denotes the third smallest degree of a non-trivial irreducible representation of $G$, and recall that expressions of type $|q+1|_{2}^{k}$ mean $\left(|q+1|_{2}\right)^{k}$.

Lemma 11.2. Let $G$ be a group such that $G / Z(G) \in\left\{\operatorname{PSL}_{n}(q), \operatorname{PSU}_{n}(q)\right\}$ with $n \geq 3, q>3$ odd and $|Z(G)|_{2}=1$. Then $|G|_{2}<\mu_{3}(G)$.

Proof. Let first $G / Z(G)=\operatorname{PSL}_{3}(q)$ with $q>3$ odd. Then $\mu_{1}(G)=q(q+1)$ by Table 1, while

$$
|G|_{2}= \begin{cases}2|q-1|_{2}^{2} & \text { if } q \equiv 1(\bmod 4), \\ 4|q+1|_{2} & \text { if } q \equiv 3(\bmod 4) .\end{cases}
$$

Thus, $|G|_{2}<\mu_{1}(G)$ unless $q-1$ is a 2-power. In the latter case,

$$
\mu_{3}(G)=\left(q^{2}-1\right)(q-1),
$$


and our claim follows. Next, let $G / Z(G)=\operatorname{PSL}_{4}(q)$ with $q>3$ odd. Then

$$
\mu_{3}(G)=\frac{\left(q^{3}-1\right)(q-1)}{2},
$$

which is larger than $|G|_{2} \leq 2(q-1)^{3}$ for $q \geq 5$. Now let $G / Z(G)=\operatorname{PSL}_{n}(q)$ with $n \geq 5$ and $q>3$ odd. Then (see Table 1 )

$$
\mu_{3}(G)=\frac{\left(q^{n}-1\right)\left(q^{n-1}-q^{2}\right)}{\left(q^{2}-1\right)(q-1)},
$$

while

$$
|G|_{2} \leq \begin{cases}(q-1)^{n-1} 2^{n-1} & \text { if } q \equiv 1(\bmod 4), \\ (q+1)^{\lfloor n / 2\rfloor} 2^{\lfloor n / 2\rfloor+n-2} & \text { if } q \equiv 3(\bmod 4) .\end{cases}
$$

Again, the claim follows.

Let $G / Z(G)=\operatorname{PSU}_{3}(q), q>3$ odd. Then $\mu_{1}(G)=q(q-1)$ by [20, Table V], while

$$
|G|_{2}= \begin{cases}2|q+1|_{2}^{2} & \text { if } q \equiv 3(\bmod 4), \\ 4|q-1|_{2} & \text { if } q \equiv 1(\bmod 4) .\end{cases}
$$

Thus, $|G|_{2}<\mu_{1}(G)$ unless $q+1$ is a 2-power. In the latter case,

$$
\mu_{3}(G)=\left(q^{2}-q+1\right)(q-1),
$$

and our claim follows. Now let $G / Z(G)=\operatorname{PSU}_{4}(q), q>3$ odd. Then

$$
\mu_{3}(G)=\frac{\left(q^{2}-q+1\right)\left(q^{2}+1\right)}{2} .
$$

Suppose first that $4 \mid(q+1)$. We have

$$
|G|_{2}=\left|\operatorname{PSU}_{4}(q)\right|_{2} \leq 2(q+1)^{3}, \quad \text { whereas } \quad \mu_{3}(G)=\frac{\left(q^{2}+1\right)\left(q^{2}-q+1\right)}{2} .
$$

So $|G|_{2}<\mu_{3}(G)$. Suppose now that $4 \mid(q-1)$. Then $|G|_{2} \leq 2(q-1)^{2}$, which is less than $\mu_{1}(G)=\frac{q^{4}-1}{q+1}$. Now assume that $G / Z(G)=\operatorname{PSU}_{n}(q)$ with $n \geq 5$. Here

$$
|G|_{2} \leq \begin{cases}(q-1)^{[(n-1) / 2]} 2^{n-1} & \text { if } q \equiv 1(\bmod 4) \\ (q+1)^{n-1} 2^{n-1} & \text { if } q \equiv 3(\bmod 4)\end{cases}
$$

As

$$
\mu_{3}(G)= \begin{cases}\frac{\left(q^{n}+1\right)\left(q^{n-1}-q^{2}\right)}{\left(q^{2}-1\right)(q+1)} & \text { if } n \text { is odd } \\ \frac{\left(q^{n}-1\right)\left(q^{n-1}+1\right)}{\left(q^{2}-1\right)(q+1)} & \text { if } n \text { is even }\end{cases}
$$

we conclude that $|G|_{2}<\mu_{3}(G)$, as required. 
Proposition 11.3. Let $G$ be a quasi-simple group such that

$$
G / Z(G) \in\left\{\operatorname{PSL}_{n}(q), \operatorname{PSU}_{n}(q)\right\} \text { with } n \geq 3 \text { and } q>3 \text { odd. }
$$

(a) If $n=3,4$, then $G$ has no $\mathrm{Syl}_{2}$-regular character.

(b) If $n \geq 5$, then $G$ has no Steinberg-like character for $p=2$.

Proof. By Lemma 2.5 (with $P=G$ ), it suffices to prove the result in the case where $|Z(G)|_{2}=1$. So we assume this, and then $|G|_{2}$ equals the order of a Sylow 2-subgroup of $G / Z(G)$.

Let first $G / Z(G)=\operatorname{PSL}_{n}(q), q>3$ odd. Let $\chi$ be a $\operatorname{Syl}_{2}$-regular character for $G$. By Lemma 11.2, we have $\chi(1)<\mu_{3}(G)$, and hence the non-trivial irreducible constituents of $\chi$ are of degree $\frac{q^{n}-1}{q-1}$ or $\frac{q^{n}-q}{q-1}$ (see Table 1). The irreducible characters of degree $\frac{q^{n}-1}{q-1}$ are induced characters $\lambda^{G}$, where $\lambda \neq 1_{P}$ is a one-dimensional character of the stabiliser $P$ of a line of the underlying space for $\mathrm{GL}_{n}(q)$, while the irreducible character of degree $\frac{q^{n}-q}{q-1}$ is the unique non-trivial constituent $\tau$ of the permutation character $1_{P}^{G}=\tau+1_{G}$ on $P$.

Let $n \geq 5$, and let $g \in \operatorname{SL}_{n}(q)$ be a block-diagonal matrix with an $(n-2) \times$ $(n-2)$-block corresponding to a primitive element of $\mathbb{F}_{q^{n-2}}$ with determinant 1 , and a $2 \times 2$-block corresponding to an element of order $q+1$. Since $g$ has no eigenvalue in $\mathbb{F}_{q}$, no conjugate of $g$ is contained in $P$, so all induced characters from $P$ to $G$ vanish on $g$. In particular, $\lambda^{G}(g)=0$ and $\tau(g)=-1$. Note that the image $\bar{g} \in G$ of $g$ has even order, so $\chi(g)=0$ if $\chi$ is Steinberg-like. Write $\chi=x_{1} 1_{G}+x_{2} \tau+\Lambda$, where $\Lambda$ is a sum of $x_{3}$-induced characters of degree $\frac{q^{n}-1}{q-1}$, with suitable $x_{i} \geq 0$. Evaluating on $g$, we see that $x_{1}=x_{2}$, but then

$$
\chi(1)=\left(x_{1}+x_{3}\right) \frac{q^{n}-1}{q-1}
$$

is divisible by some odd prime, so cannot equal the 2-power $|G|_{2}$. This proves part (b) for $G / Z(G)=\operatorname{PSL}_{n}(q)$.

Now assume that $n=4$. Then an easy estimate shows that, when $q-1$ is not a 2-power, so $|q-1|_{2} \leq \frac{q-1}{3}$, and $q \neq 7$, then $|G|_{2}<\mu_{1}(G)$. So we may assume that, in addition, either $q=7$ or $q-1$ is a power of 2 . For $q \neq 7$, let $g$ be the 2-element

$$
g=\left(\begin{array}{cccc}
0 & 1 & 0 & 0 \\
a & 0 & 0 & 0 \\
0 & 0 & 0 & 1 \\
0 & 0 & a^{-1} & 0
\end{array}\right) \in \operatorname{SL}_{4}(q),
$$

where $a \in \mathbb{F}_{q}^{\times}$is a 2-element of order $q-1$. Observe that, again, $g$ is not conjugate to an element of $P$, and thus $\lambda^{G}(h)=0$ and $\tau(h)=-1$. As $g$ is a 2-element 
and $\chi$ is $\operatorname{Syl}_{2}$-regular, we have $\chi(g)=0$. We may now argue as above to conclude. When $q=7$, the candidate characters have degrees 1, 399 and 400, while $|G|_{2}=2^{9}=512$, so clearly there can be no $S_{1} l_{2}$-regular character.

Now consider the case when $G / Z(G)=\operatorname{PSL}_{3}(q)$. The proof of Lemma 11.2 shows that $\mu_{1}(G)>|G|_{2}$ unless $q-1$ is a 2-power. In the latter case, the possible constituents of $\chi$ can have degrees $1, q^{2}+q, q^{2}+q+1$, while $|G|_{2}=2(q-1)^{2}$. Clearly, at most one of the degrees $q^{2}+q, q^{2}+q+1$ can contribute to $\chi(1)$, but then necessarily $q=5$. But, in that case, the character table shows that there is no $\mathrm{Syl}_{2}$-regular character. This completes the proof of (a) when $G / Z(G)=\operatorname{PSL}_{n}(q)$.

Now let $G / Z(G)=\operatorname{PSU}_{n}(q)$ with $q>3$ odd, and let $\chi$ be a Syl 2 -regular character of $G$. According to Lemma 11.2, $\chi(1)<\mu_{3}(G)$, and hence the non-trivial irreducible constituents of $\chi$ are of degree (see [20, Table V])

$$
\frac{q^{n}-(-1)^{n}}{q+1} \text { or } \frac{q^{n}+(-1)^{n} q}{q+1} .
$$

The first of these are semisimple characters lying in the Lusztig series of an element $s$ of order $q+1$ in the dual group

$$
G^{*}=\operatorname{PGU}_{n}(q) \text { with centraliser } C_{G^{*}}(s) \cong \mathrm{GU}_{n-1}(q) ;
$$

the second is a unipotent character, $\tau$ say, corresponding to the character of the Weyl group $S_{n}$ parameterised by the partition $(n-1,1)$. Let $g \in \mathrm{SU}_{n}(q)$ be a regular element of even order in a maximal torus $T$ of order (see [10, Lemma 3.1 (a)])

$$
\frac{\left(q^{2}-1\right)\left(q^{2 n-2}-(-1)^{2 n-2}\right)}{q+1} .
$$

Then no conjugate of the dual maximal torus $T^{*}$ contains $s$, so the characters in $\mathcal{E}(G, s)$ vanish on $g$ (see, e.g., [11, Proposition 6.4]). If $\chi$ is Steinberg-like, then $\chi(g)=0$. As $\tau$ is unipotent, its value on $g$ is (up to sign) the same as $\psi(h)$, where $\psi \in \operatorname{Irr}\left(S_{n}\right)$ is labelled by $(n-1,1)$ and $h$ is a permutation of cycle shape $(n-2,2)$; see [10, Proposition 3.3 and the remark before Proposition 4.2]. The Murnaghan-Nakayama rule gives $\psi(h) \in\{ \pm 1\}$, so $\tau(g) \in\{ \pm 1\}$. We may now argue as in the first part to conclude that $\chi$ cannot be Steinberg-like, thus completing the proof of (b).

Next, assume that $G / Z(G)=\operatorname{PSU}_{4}(q)$ with $q>3$ odd. If $q+1$ is not a power of 2 and $q \neq 5$, 9, then $|G|_{2}<\mu_{1}(G)$ as $|q+1|_{2} \leq \frac{q+1}{3}$. So now assume that $q+1$ is a power of 2 , and hence in particular $q \equiv 3(\bmod 4)$. Then we have $|G|_{2}=2(q+1)^{3}$, while the three smallest character degrees are $1, \frac{q^{4}-1}{q+1}, \frac{q^{4}+q}{q+1}$, with the trivial character occurring at most once. It is easily seen that there is no non-negative integral solution for a possible decomposition of $\chi$. When $q=5$, the 
three smallest degrees are $1,104,105$, while $|G|_{2}=128$; if $q=9$, then the three smallest degrees are 1,656,657, while $|G|_{2}=512$; so in neither case can there be $\mathrm{Syl}_{2}$-regular characters either.

Finally, when $G / Z(G)=\operatorname{PSU}_{3}(q)$, again the proof of Lemma 11.2 shows that $q+1$ must be a 2-power. Here the possible constituents of $\chi$ have degrees 1 , $q^{2}-q, q^{2}-q+1$, while $|G|_{2}=2(q+1)^{2}$. Again, an easy consideration shows that at most the case $q=7$ needs special attention. But there, the existence of $\mathrm{Syl}_{2}$-regular characters can be ruled out from the known character table.

We now treat the case $q=3$, which is considerably more delicate.

Lemma 11.4. Let $G=\mathrm{PSL}_{3}(3)$ or $\mathrm{PSU}_{3}(3)$. Then $G$ does not have reducible $\mathrm{Syl}_{2}$-regular characters.

Proof. For $G=\operatorname{PSL}_{3}(3)$, we have $|G|_{2}=16$, and all irreducible characters of degree less than 16 take non-negative values on class $4 \mathrm{~A}$, so there are no $\mathrm{Syl}_{2}$-regular characters. For $G=\operatorname{PSU}_{3}(3)$, we have $|G|_{2}=32$, and all irreducible characters have degree at most that large. Since the smallest non-trivial character degree is 6 , those of degrees 27 and 28 cannot be constituents of a $\mathrm{Syl}_{2}$-regular character $\chi$. Thus, we need to consider the characters of degrees $1,6,7,14,21$. Clearly, those of degree 21 cannot occur either. As $32 \equiv 4(\bmod 7)$, we see that the character of degree 6 has to appear at least three times, but then the values on elements of order 4 give a contradiction.

Remark 11.5. $\mathrm{PSL}_{3}(3)$ and $\mathrm{PSU}_{3}(3)$ both have irreducible $\mathrm{Syl}_{2}$-regular characters; see Proposition 3.1.

Lemma 11.6. Let $G=\mathrm{PSL}_{4}(3)$ or $\mathrm{PSU}_{4}(3)$. Let $\chi$ be a $\mathrm{Syl}_{2}$-vanishing character of $G$. Then $l_{2}(\chi) \geq 4$.

Proof. Suppose the contrary. Note that $\chi$ is reducible. As $|G|_{2}=128$, we have $\chi(1) \leq|G|_{2} \cdot 3=384$. Let $\tau$ be an irreducible constituent of $\chi$ of maximal degree. For all numerical data, see [1].

Let $G=\mathrm{PSL}_{4}$ (3). Then we have $\tau(1)<351$ (otherwise, we have $\tau(1)=351$, so $(\chi-\tau)(1)=33$, and then $\chi(2 \mathrm{~B})>0$, which is false).

Let $\mu \in \operatorname{Irr}(G)$ and $\mu(1)<351$. Then we have $\mu(4 \mathrm{~B}) \geq 0$ unless $\mu(1)=90$, and $\mu(4 \mathrm{~B}) \neq 0$ unless $\mu(1)=52$ or 260 . Let $\mu(1)=90$. Then $(\chi, \mu)>0$. Indeed, otherwise, the irreducible constituents of $\chi$ are of degree 52 or 260, which implies $\chi(2 \mathrm{~A})>0$, a contradiction.

It follows that $(\chi-\mu)(1) \leq 294$. Let $\sigma \in \operatorname{Irr}(G)$ with $\sigma(1)=39$. The irreducible characters of $G$ of degree at most 294 and distinct from $\sigma$ are non-negative 
at $2 \mathrm{~A}$. In addition, $\sigma(2 \mathrm{~A})=-1$, and $\mu(2 \mathrm{~A})=10$. As $\chi(2 \mathrm{~A})=0$, it follows that $(\chi, \sigma) \geq 10$. Then $\chi(1) \geq \mu(1)+10 \sigma(1)>384$, a contradiction.

Let $G=\mathrm{PSU}_{4}(3)$ and $\mu \in \operatorname{Irr}(G)$ with $(\chi, \mu)>0$. The irreducible characters of degree at most 384 are of degree at most 315. If $\tau(1)=315$, then $\mu(1) \leq 69$. Then $\tau(2 \mathrm{~A})>0$ and $\mu(2 \mathrm{~A})>0$, a contradiction.

Suppose that $\tau(1)=280$. Then $\mu(1) \leq 104$, but then we obtain a positive value on class $2 \mathrm{~A}$. The same consideration rules out $\tau(1)=210$.

Suppose that $\tau(1)=189$. It occurs once as otherwise $1_{G}$ occurs 6 times, which is false as $\left(\chi, 1_{G}\right) \leq 3$. Then $\mu(1) \neq 140,90$, so $\mu(4 \mathrm{~A})>0, \tau(4 \mathrm{~A})>0$, a contradiction. No other option exists as the irreducible characters of degree less than 189 are positive on $2 \mathrm{~A}$.

Lemma 11.7. Let $\mathrm{PSL}_{4}(3) \leq G \leq \mathrm{PGL}_{4}(3)$ or $\mathrm{PSU}_{4}(3) \leq G \leq \mathrm{PGU}_{4}(3)$. Let $\chi$ be a 2-vanishing character of $G$. Then $l_{2}(\chi) \geq 4$.

Proof. By Lemma 2.9 (a), $\chi=\psi^{G}$, where $\psi$ is a proper character of $G^{\prime}$. By inspection of the character table of $G^{\prime}$ (see [1]) it is easily checked that the conjugacy class of any element $g \in G^{\prime}$ of 2-power order is $G$-invariant. Then, by Lemma $2.9(\mathrm{~b}), \psi(g)=0$ for every 2-element $g \neq 1$ of $G^{\prime}$. This means that $\psi$ is $\mathrm{Syl}_{2}$-vanishing. By Lemma $11.6, l_{2}(\psi) \geq 4$. Then $l_{2}\left(\psi^{G}\right) \geq 4$.

Lemma 11.8. Let $G=\mathrm{PGL}_{4}(3)$, and let $\chi$ be a 2-vanishing character of $G$. Let $\eta_{1}, \ldots, \eta_{k}$ be the irreducible constituents of $\chi$ disregarding multiplicities, and $\eta=\eta_{1}+\cdots+\eta_{k}$. Then $\eta(1) \geq 2|G|_{2}$.

Proof. Let $G^{\prime}=\mathrm{PSL}_{4}(3)$. Then $\left|G^{\prime}\right|_{2}=128$, and thus $2 \cdot|G|_{2}=512$. Suppose the contrary. Then we have $\eta(1)<512$. We can assume that $\eta_{i}(1) \geq \eta_{j}(1)$ for $1 \leq i<j \leq k$. Note that $k>1$; otherwise, $\chi=a \eta_{1}$ for some $a$, and hence $\eta_{1}$ is a 2-vanishing character of $G$, and $\eta_{1}(1)$ is a multiple of $|G|_{2}$. By [1], $G$ has no character of degree at most 512 with this property.

By [1], we have $\eta_{1}(1) \leq 468$. Note that all irreducible characters of $G^{\prime}$ of degree at most 468 extend to $G=G^{\prime} \cdot 2$ except $\chi_{11}, \chi_{12}$ of degree $260, \chi_{9}, \chi_{10}$ of degree $234, \chi_{6}, \chi_{7}$ of degree 65 and $\chi_{2}, \chi_{3}$ of degree 26 . The corresponding characters of $G$ are of degrees 520, 468, 130 and 52, respectively. Let $\chi=\sum a_{i} \eta_{i}$, where $a_{i}>0$ are integers.

(i) Suppose $\eta_{1}(1)=468$. Then $\sum_{i>1} \eta_{i}(1) \leq 44$. Computing $\chi(4 \mathrm{~B})$, we get a contradiction (as $\eta_{1}(4 \mathrm{~B})=0$ and $\sum_{i>1} a_{i} \eta_{i}(4 \mathrm{~B})>0$ and $k>1$ ).

(ii) Suppose $\eta_{1}(1)=416$. If $\eta_{2}(1) \leq 90$, then $\sum_{i>2} \eta_{i}(1) \leq 6$, whence $k=3$ and $\eta_{3}=1_{G}$. Computing $\chi(2 \mathrm{~B})$, we get a contradiction.

So $\eta_{2}(1) \leq 52$. If $\eta_{2}(1)=52$, then $\sum_{i>2} \eta_{i}(1) \leq 44$. Computing $\chi(4 \mathrm{~B})$, we get a contradiction unless $k=2$ and $\eta_{2}(4 \mathrm{~B})=0$ (that is, $\eta_{2}=\chi_{5}$ in [1]). In this 
case, computing $\chi(4 C)$ gives a contradiction. So $\eta_{i}(1) \leq 39$ for $i>1$. This violates $\chi(2 \mathrm{~B})=0$.

(iii) Let $\eta_{1}(1)=390$. Then $\eta_{2}(1) \leq 90$. If $\eta_{2}(1)=90$, then $\eta_{3}(1) \leq 32$, whence $k=1$ and $\eta_{3}(1)=1$. This conflicts with $\chi(4 \mathrm{~A})=0$. If $\eta_{2}(1) \leq 52$, then computing $\chi(4 \mathrm{~B})$ yields a contradiction.

(iv) Let $\eta_{1}(1)=351$. So we have $\sum_{i>1} \eta_{i}(1) \leq 161$. If $\eta_{2}(1)=130$, then $\sum_{i>1} \eta_{i}(1) \leq 31$, and hence $\eta_{3}(1)=1$, a contradiction with $\chi(20 \mathrm{~A})=0$. Let $\eta_{2}(1) \leq 90$. Then $\eta_{i}(1) \leq 71$ for $i>2$. Then we have $\eta_{i}(2 \mathrm{~A})+\eta_{i}(2 \mathrm{~B})>0$ for $i=1, \ldots, k$, which violates $\chi(2 \mathrm{~A})+\chi(2 \mathrm{~B})=0$.

(v) Let $\eta_{1}(1)=260$. Then $\eta_{1}(2 \mathrm{~A}) \geq 0$, and the only irreducible character $\lambda$ of degree less than 260 with negative value at $2 \mathrm{~A}$ has $\lambda(1)=39$. It follows that $(\chi, \lambda) \geq 0$, and then $\eta_{i}(1) \leq 213$ if $i>1$ and $\eta_{i} \neq \lambda$. Note that $\lambda(8 \mathrm{~A})=1$ and $\eta_{1}(8 \mathrm{~A})=0$. As $\chi(8 \mathrm{~A})=0$, it follows that a character of degree 130 occurs in $\chi$, which implies $\eta_{2}(1)=130$. Then we get a contradiction to $\chi(2 \mathrm{~A})+\chi(4 \mathrm{~B})=0$.

(vi) Let $\eta_{1}(1) \leq 234$. Computing $\chi(2 \mathrm{~A})+\chi(4 \mathrm{~B})$ leads to a contradiction.

Lemma 11.9. Let $G=\mathrm{PGU}_{4}(3)$, and let $\chi$ be a 2-vanishing character of $G$. Let $\eta_{1}, \ldots, \eta_{k}$ be the irreducible constituents of $\chi$ disregarding multiplicities, and $\eta=\eta_{1}+\cdots+\eta_{k}$. Then $\eta(1) \geq 2|G|_{2}$.

Proof. Let $G^{\prime}=\operatorname{PSU}_{4}(3)$. Then $\left|G^{\prime}\right|_{2}=128,|G|_{2}=512$ and $2 \cdot|G|_{2}=1024$. Suppose the contrary. Then $\eta(1)<1024$. We can assume that $\eta_{i}(1) \geq \eta_{j}(1)$ for $1 \leq i<j \leq k$.

Note that $\chi \cdot \tau=\chi$ for every linear character $\tau$ of $G$. Therefore, $\eta_{i} \tau$ is a constituent of $\eta$. Let $g \in G \backslash G^{\prime}$. Then $\eta_{i} \tau=\eta$ implies $\eta_{i}(g)=0$.

By [1], if $630 \neq \eta_{i}(1)>420$, then $\eta_{i}(4 E) \neq 0$ or $\eta_{i}(4 G) \neq 0$; it follows that $\eta$ must contain at least 2 representations of the same degree, which contradicts $\eta(1) \leq 1024$.

So $\eta_{i}(1)$ either equals 630 or $\eta_{i}(1) \leq 420$. By [1], $\eta_{i}(2 \mathrm{~A})+\eta_{i}(4 \mathrm{~B})>0$ for these $\eta_{i}$ unless $\eta_{i}(1)=210$. This violates $\chi(2 \mathrm{~A})+\chi(4 \mathrm{~B})=0$ unless $k=1$ and $\eta_{1}(1)=210$. Then $\chi(2 \mathrm{~A})>0$, a contradiction.

Lemma 11.10. Let $H=H_{1} \times \cdots \times H_{n}$, where $H_{1} \cong \cdots \cong H_{n} \cong \mathrm{PGL}_{4}(3)$ or $\mathrm{PGU}_{4}(3)$. Let $\chi$ be a 2-vanishing character of $H$. Then $\chi(1) \geq 2^{n+1}|H|_{2}$.

Proof. By Lemma 11.7, the claim holds for $n=1$, so, by induction, we can assume that it is true for $X:=H_{2} \times \cdots \times H_{n}$. By Lemma 2.7, $\chi=\sum_{i} \eta_{i} \sigma_{i}$, where $\eta_{i} \in \operatorname{Irr}\left(H_{1}\right), \sigma_{i}$ are 2-vanishing characters of $X$ and $\chi^{\prime}=\sum_{i} l_{2}\left(\sigma_{i}\right) \eta_{i}$ is a 2-vanishing character of $H_{1}$. By induction, $\sigma_{i}(1) \geq 2^{n}|X|_{2}$. By Lemmas 11.8 and 11.9 applied to $\chi^{\prime}$, we have $\sum_{i} \eta_{i}(1) \geq 2\left|H_{1}\right|_{2}$, so $\chi(1) \geq 2^{n+1}|H|_{2}$ by Lemma 2.9. 
Proposition 11.11. Let $n>1$ and $G=\mathrm{GL}_{4 n}(3)$ or $\mathrm{GU}_{4 n}(3)$. Let $\chi$ be a 2-vanishing character of $G$. Then $l_{2}(\chi) \geq 4$.

Proof. Let $X$ be the direct product of $n$ copies of $\mathrm{GL}_{4}(3)$ or $\mathrm{GU}_{4}(3)$. Let $\chi$ be a 2-vanishing character of $X$. By Lemmas 2.5 and 11.10, $\chi(1) \geq 2^{n+1}|X|_{2}$.

Let $Y=X \cdot S_{n}$, the semidirect product, where $S_{n}$ acts on $X$ by permuting the factors. Then $Y$ contains a Sylow 2-subgroup of $G$. Let $M=X \cdot S$, where $S \in \operatorname{Syl}_{2}\left(S_{n}\right)$, so the index $|G: M|$ is odd. Note that $|G|_{2}=|X|_{2} \cdot\left|S_{n}\right|_{2}$. As $\left|S_{n}\right|_{2} \leq 2^{n-1}$ (see the proof of Proposition 6.7), the result follows for these groups.

Theorem 11.12. Let $p=2, m>3$ and $G$ be one of $\mathrm{GL}_{m}(3), \mathrm{SL}_{m}(3), \mathrm{PSL}_{m}(3)$, $\mathrm{GU}_{m}(3), \mathrm{SU}_{m}(3), \mathrm{PSU}_{m}(3)$. Then $G$ has no Steinberg-like character. Moreover, if $\chi$ is a 2-vanishing character of $G$, then $l_{2}(\chi) \geq 4$.

Proof. Let first $G=\mathrm{GL}_{m}(3)$ or $\mathrm{GU}_{m}(3)$. For $m \equiv 0(\bmod 4)$, the result is stated in Proposition 11.11. Let $m=4 n+l$, where $1 \leq l<4$, and $H=\mathrm{GL}_{4 n}$ (3) or $\mathrm{GU}_{4 n}$ (3). Let $S_{0}$ be a Sylow 2-subgroup of $\mathrm{GL}_{l}(3)$ or $\mathrm{GU}_{l}(3)$; set $U=H \times S_{0}$. Then $U$ contains a Sylow 2-subgroup of $G$. Therefore, we have $l_{2}(\chi)=l_{2}\left(\left.\chi\right|_{U}\right)$. By Lemma 2.5, if $v$ is a 2-vanishing character of $U$, then $l_{2}(v)=l_{2}(\mu)$ for some 2-vanishing character $\mu$ of $H$. So $l_{2}(v) \geq 4$ by Proposition 11.11. So the result follows for these groups. For $G=\mathrm{SL}_{m}$ (3) or $\mathrm{SU}_{m}$ (3), the result follows from the above and Lemma 2.12. For $G=\mathrm{PSL}_{m}(3)$ or $\mathrm{PSU}_{m}$ (3), the statement follows from the above and Lemma 2.5.

\subsection{Orthogonal and symplectic groups at $p=2$}

Let $V$ be the natural module for $H=\operatorname{Sp}_{2 n}(q), q$ odd, and for $g \in G$, let $d(g)$ be the dimension of the fixed point subspace of $g$ on $V$. Let $\omega_{n}$ denote the Weil character of $H$. By Howe [7, Proposition 2], $\left|\omega_{n}(g)\right|=q^{d(g) / 2}$. Let $\omega_{n}=\omega_{n}^{\prime}+\omega_{n}^{\prime \prime}$, where $\omega_{n}^{\prime}, \omega_{n}^{\prime \prime} \in \operatorname{Irr}(H)$ and $\omega_{n}^{\prime}(z)=-\omega_{n}^{\prime}(1)$ for $1 \neq z \in Z(H)$.

Lemma 11.13. The following statements hold.

(a) Let $h \in H$ be semisimple such that $h$ and $z h$ fix no non-zero vector on $V$. Then $\left|\omega_{n}^{\prime \prime}(h)\right| \leq 1$.

(b) Let $V=V_{1} \oplus V_{2}$, where $V_{1}$ is a non-degenerate subspace of dimension 2, and let $g \in H$ be an element such that $g V_{i}=V_{i}, i=1,2,\left.g\right|_{V_{1}}=-\mathrm{Id}$, and $g$ and $z g$ fix no non-zero vector on $V_{2}$. Then $\left|\omega_{n}^{\prime \prime}(g)\right| \geq \frac{q-1}{2}$.

(c) Let $q>3$. Then $\omega_{n}^{\prime \prime}$ is not constant on the 2-singular elements of $\operatorname{PSp}_{2 n}(q)$. 
Proof. (a) We have

$$
\omega_{n}(h)=\omega_{n}^{\prime}(h)+\omega_{n}^{\prime \prime}(h) \text { and } \omega_{n}(z h)=-\omega_{n}^{\prime}(h)+\omega_{n}^{\prime \prime}(h) .
$$

Therefore, by [7, Proposition 2], $2 \geq\left|\omega_{n}(h)+\omega_{n}(z h)\right|=\left|2 \omega_{n}^{\prime \prime}(h)\right|$, whence the claim.

(b) By [7, Proposition 2], we have

$$
\left|\omega_{n}(g)\right|=1 \quad \text { and } \quad\left|\omega_{n}(z g)\right|=q .
$$

Then $q-1 \leq\left|\omega_{n}(g)+\omega_{n}(z g)\right|=\left|2 \omega_{n}^{\prime \prime}(g)\right|$, whence the claim.

(c) Choose $g$ as in (b) and $h$ to be an element stabilising $V_{1}, V_{2}$ such that $h$ coincides with $g$ on $V_{2}$ and the matrix of $h$ on $V_{1}$ is similar to $\left(\begin{array}{rr}0 & 1 \\ -1 & 0\end{array}\right)$. Then $h$ is a 2-singular element satisfying (a), and hence $\left|\omega_{n}^{\prime \prime}(h)\right| \leq 1$. Let $\bar{h}$ and $\bar{g}$ be the images of $h, g$ in $H / Z(H)$. Then $\bar{h}$ and $\bar{g}$ are 2-singular elements of $\operatorname{PSp}_{2 n}(q)$. As $Z(H)$ is in the kernel of $\omega_{n}^{\prime \prime}$, this can be viewed as a character of $H / Z(H)$. As $\frac{q-1}{2}$ is greater than 1 for $q>3$, (c) follows.

Proposition 11.14. Let $G=\operatorname{PSp}_{2 n}(q)$ with $q>3$ odd and $n \geq 2$. Then $G$ has no $\mathrm{Syl}_{2}$-regular characters.

Proof. By [19, Table 2], we have

$$
\left|\operatorname{PSp}_{2 n}(q)\right|_{2}= \begin{cases}|q-1|_{2}^{n} \cdot 2^{n-1} \cdot|n !|_{2} & \text { if } 4 \mid(q-1), \\ |q+1|_{2}^{n} \cdot 2^{n-1} \cdot|n !|_{2} & \text { if } 4 \mid(q+1)\end{cases}
$$

We have seen in the proof of Proposition 6.9 (b) that $|n !|_{2} \leq 2^{n-1}$. So we have

$$
\left|\operatorname{PSp}_{2 n}(q)\right|_{2} \leq \begin{cases}|q-1|_{2}^{n} \cdot 4^{n-1} & \text { if } 4 \mid(q-1), \\ |q+1|_{2}^{n} \cdot 4^{n-1} & \text { if } 4 \mid(q+1) .\end{cases}
$$

On the other hand, $\mu_{3}(G)=\frac{\left(q^{n}-1\right)\left(q^{n}-q\right)}{2(q+1)}$ by [20, Theorem 5.2], and this is larger than $|G|_{2}$ unless $n=2$ and $q=5,7$. We set aside these cases for a moment. Then, otherwise, if $\chi$ is $\mathrm{Syl}_{2}$-regular, the constituents of $\chi$ are either Weil characters or the trivial character. Now note that a Weil character of $\operatorname{Sp}_{2 n}(q)$ of degree $\frac{q^{n} \pm 1}{2}$ has the centre in its kernel if and only if its degree is odd. So the non-trivial constituents of $\chi$ have degree $\frac{q^{n}-1}{2}$ if $q \equiv 3 \bmod 4$ and $n$ is odd, and $\frac{q^{n}+1}{2}$ otherwise. According to Lemma 2.1, the trivial character occurs at most once in $\chi$. As $\frac{q^{n} \pm 1}{2}$ is never a power of 2 for $n \geq 2$ and odd $q$ (consider a Zsigmondy prime divisor), the trivial character must occur exactly once. Let $\psi_{1}, \psi_{2}$ denote the two Weil characters of $G$, interchanged by the outer diagonal automorphism $\gamma$ of $G$. Observe that $\gamma$ is induced by an element of $\operatorname{GL}_{2 n}(q)$ and thus fixes all involution 
classes of $G$. Let $g \in G$ be an involution, and write $a:=\psi_{1}(g)=\psi_{2}(g)$. Then $\chi(g)=m a+1$, where $m$ is the number of non-trivial constituents of $\chi$. As necessarily $m>1$ (compare the degrees), we see that $\chi(g) \neq 0$, so $\chi$ is not $\mathrm{Syl}_{2}$-regular.

We now discuss the two exceptions. For $G=\operatorname{PSp}_{4}(5),|G|_{2}=2^{6}=64$, and all irreducible characters of degree at most 64 take non-negative values on class $2 \mathrm{~B}$, so there is no $\operatorname{Syl}_{2}$-regular character. For $G=\operatorname{PSp}_{4}(7),|G|_{2}=2^{8}=256$, and all irreducible characters of degree at most 256 take positive values on class $8 \mathrm{~A}$, except for one of degree 175 which takes value -1 , and one of degree 224 which takes value 0 . As at most one of those latter two characters could occur, and at most once, there can be no $\mathrm{Syl}_{2}$-regular character for $p=2$.

Proposition 11.15. Let $G=\Omega_{2 n+1}(q)$ with $q>3$ odd and $n \geq 3$. Then $G$ has no $\mathrm{Syl}_{2}$-regular characters.

Proof. According to [20, Theorem 6.1], we have $\mu_{1}(G)=\frac{q^{2 n}-1}{q^{2}-1}$, which is larger than $|G|_{2}$, unless either $n=3$ and $q=7$, or $n=4$ and $q=5,7$.

For $G=\Omega_{7}(7)$, the only non-trivial character of degree less than $|G|_{2}=2^{12}$ is the semisimple character of degree 2451 (see [20]). Since the trivial character can occur at most once in a $\mathrm{Syl}_{2}$-regular character, we see that no example can arise here. For $G=\Omega_{9}(5)$, the only non-trivial character of degree less than $|G|_{2}=2^{14}$ is the semisimple character of degree 16276 (see [20]). Again, this does not lead to an example. For $G=\Omega_{9}(7)$, the only non-trivial character of degree less than $|G|_{2}=2^{18}$ is the character of degree 120100 , and we conclude as before.

Proposition 11.16. Let $G=\mathrm{P} \Omega_{2 n}^{ \pm}(q)$ with $q>3$ odd and $n \geq 4$. Then $G$ has no $\mathrm{Syl}_{2}$-regular characters.

Proof. The second smallest non-trivial character degree of $G=\mathrm{P} \Omega_{2 n}^{+}(q)$ is given by $\mu_{2}(G)=\frac{\left(q^{n}-1\right)\left(q^{n-1}-1\right)}{2(q+1)}$ (see [16, Theorem 1.4]), which is larger than $|G|_{2}$, unless $(n, q)=(4,7)$. Leaving that case aside for a moment, we see that any $\mathrm{Syl}_{2}$-regular character of $G$ is a multiple of the smallest non-trivial character, of degree $\frac{\left(q^{n}-1\right)\left(q^{n-1}+q\right)}{\left(q^{2}-1\right)}$, plus possibly the trivial character. Arguing as in the case of symplectic groups, we see that such characters take non-zero value on involutions. For $G=\mathrm{P} \Omega_{8}^{+}(7)$, the constituents of a $\mathrm{Syl}_{2}$-regular character could have degree 1,17500 , or 51300 . No non-negative integral linear combination of these three degrees, with $1_{G}$ appearing at most once, adds up to $|G|_{2}=2^{16}=65536$.

The second smallest non-trivial character degree of $G=\mathrm{P} \Omega_{2 n}^{-}(q)$ is (see again [16, Theorem 1.4])

$$
\mu_{2}(G)=\frac{\left(q^{n}+1\right)\left(q^{n-1}+1\right)}{2(q+1)},
$$

which is larger than $|G|_{2}$. We conclude as before. 
Again, we are left with the case that $p=2, q=3$.

Lemma 11.17. Let $G=\mathrm{PSp}_{6}(3), \Omega_{7}(3)$ or $\mathrm{P} \Omega_{8}^{-}(3)$. Then $G$ has no Steinberglike character.

Proof. For $G=\operatorname{PSp}_{6}(3)$, we have $|G|_{2}=2^{9}$, and all irreducible characters of $G$ of degree at most 512 take positive value on the class $4 \mathrm{~A}$; see [1].

Let $G=\Omega_{7}$ (3). Then $|G|_{2}=2^{9}$. All irreducible characters of $G$ of at most that degree are positive at the elements of conjugacy class $2 \mathrm{~B}$ [1].

Let $G=\mathrm{P} \Omega_{8}^{-}$(3). By [1], $G$ has 8 irreducible characters of degree at most $|G|_{2}=2^{10}$. All of them take positive values on class $2 \mathrm{~A}$. So the result follows.

Lemma 11.18. Let $G=\mathrm{PGO}_{8}^{+}(3)$ or $\mathrm{PSp}_{8}(3)$. Let $\chi$ be a 2-vanishing character of $G$, and $\eta_{1}, \ldots, \eta_{k}$ the irreducible constituents of $\chi$ disregarding their multiplicities. Set $\eta=\eta_{1}+\cdots+\eta_{k}$. Then $\eta(1) \geq 2|G|_{2}$.

Proof. Suppose first that $G=\mathrm{PGO}_{8}^{+}(3)$. Note that $\left|G^{\prime}\right|_{2}=2^{12}$, so

$$
2 \cdot|G|_{2}=2^{15}=32768 \text {. }
$$

Suppose the contrary. Then $\eta(1)<32768$. We use notation from [1]. There are 43 characters of $G^{\prime}$ of degree less than 32768 ; the maximal degree among them is 29120.

There is only one irreducible character of $G^{\prime}$ of degree less that 32768 that is negative at 4A (this is of degree 9450), while all other are positive. So it must be a constituent of $\left.\eta\right|_{G^{\prime}}$. This character extends to $G$, so the other constituents of $\eta$ are of degrees at most $32768-9450=23318$.

It follows that $\eta_{i}(1) \leq 18200$. In fact, $\eta_{i}(1)<18200$. Indeed, if $\eta_{i}(1)=18200$ and $\eta_{j}(1)=9450$ for some $i \neq j$, then $\eta_{l}$ for $l \neq i, j$ are of degree at most $23318-18200=5118$. These characters are positive at $2 \mathrm{~A}$ (as well as those of degree 18200 and 9450). This violates $\chi(2 \mathrm{~A})=0$.

Thus, $\eta_{i}(1)<18200$, and hence $\eta_{i}(1) \leq 17550$. Furthermore, computing the character table of $G$ by a program in the computer package GAP, one observes that there are 4 distinct irreducible characters of degree 17550 , and only one irreducible character of this degree for $G^{\prime}$. It follows that these 4 characters differ from each other by multiplication by a linear character. As $\left|G / G^{\prime}\right|=4$, one observes that $\chi \cdot \lambda=\chi$ for every linear character $\lambda$ of $G$. Therefore, $\eta_{i} \cdot \lambda$ must be a constituent of $\chi$. So, if $\eta_{i}(1)=17550$, then there are 3 more constituents of $\eta$ of this degree, which contradicts the inequality $\eta(1)<32768$.

Thus, $\eta_{i}(1)<17550$ for $i=1, \ldots, k$. By [1], all such irreducible characters of $G^{\prime}$, and hence of $G$, are positive at $4 \mathrm{~A}$, which contradicts $\chi(4 \mathrm{~A})=0$. 
Let $G=\operatorname{PSp}_{8}(3)$. Note that $|G|_{2}=2^{14}$, so $2 \cdot|G|_{2}=2^{15}=32768$. Suppose the contrary. Then $\eta(1)<32768$. There are 19 irreducible characters of degree less than 32768 . All such characters are positive at $4 \mathrm{~A}$, which violates $\chi(4 \mathrm{~A})=0$.

Lemma 11.19. The following statements hold.

(a) Let $H=H_{1} \times \cdots \times H_{n}$, where $H_{1} \cong \cdots \cong H_{n} \cong \mathrm{PGO}_{8}^{+}$(3) or $\mathrm{PSp}_{8}$ (3). Let $\chi$ be a 2-vanishing character of $H$. Then $\chi(1) \geq 2^{n}|H|_{2}$.

(b) Let $G=G_{1} \times \cdots \times G_{n}$, where $G_{1} \cong \cdots \cong G_{n} \cong \mathrm{GO}_{8}^{+}(3)$ or $\mathrm{Sp}_{8}(3)$. Let $\chi$ be a 2-vanishing character of $G$. Then $\chi(1) \geq 2^{n}|G|_{2}$.

Proof. (a) If $n=1$, then the result is contained in Lemma 11.18. By induction, we can assume that it is true for $X:=H_{2} \times \cdots \times H_{n}$. By Lemma 2.7, $\chi=\sum_{i} \eta_{i} \sigma_{i}$, where $\eta_{i} \in \operatorname{Irr}\left(H_{1}\right), \sigma_{i}$ are 2-vanishing characters of $X$ and $\chi^{\prime}=\sum l_{2}\left(\sigma_{i}\right) \eta_{i}$ is a 2-vanishing character of $H_{1}$. By induction, $\sigma_{i}(1) \geq 2^{n-1}|X|_{2}$. By Lemma 11.18 applied to $\chi^{\prime}$, we have $\sum_{i} \eta_{i}(1) \geq 2\left|H_{1}\right|_{2}$, so $\chi(1) \geq 2^{n}|H|_{2}$ by Lemma 2.9.

(b) This follows from (a) and Lemma 2.5 as $Z(G)$ is a 2-group.

Lemma 11.20. Let $G=\mathrm{GO}_{8 n}^{+}(3), \Omega_{8 n}^{+}(3), \mathrm{P}_{8 n}^{+}(3), \mathrm{Sp}_{8 n}(3)$ or $\mathrm{PSp}_{8 n}$ (3). Then $G$ has no Steinberg-like character for $p=2$.

Proof. Let $X$ be the direct product of $n$ copies of $\mathrm{GO}_{8}^{+}(3)$ or $\mathrm{Sp}_{8}(3)$. Let $v$ be a 2-vanishing character of $X$. By Lemma 11.10, $v(1) \geq 2^{n}|X|_{2}$.

Let $Y=X \cdot S_{n}$, the semidirect product, where $S_{n}$ acts on $X$ by permuting the factors. Then $Y$ contains a Sylow 2-subgroup of $G$. Let $M=X \cdot S$, where $S \in \operatorname{Syl}_{2}\left(S_{n}\right)$, so the index $|G: M|$ is odd. Note that $|G|_{2}=|X|_{2} \cdot\left|S_{n}\right|_{2}$. As $\left|S_{n}\right|_{2} \leq 2^{n-1}$ (see the proof of Proposition 6.7), the result follows for the groups $\mathrm{GO}_{8 n}^{+}$(3) and $\mathrm{Sp}_{8 n}$ (3). For $G=\Omega_{8 n}^{+}(3)$, the result follows from the above and Lemma 2.12. For $G=\mathrm{P}_{8 n}^{+}$(3) or $\mathrm{PSp}_{8 n}$ (3), the statement follows from the above and Lemma 2.5.

Proposition 11.21. Let $m \geq 4$ and $G=\mathrm{GO}_{2 m}^{+}$(3) or $\mathrm{Sp}_{2 m}$ (3). Then $G$ has no Steinberg-like character for $p=2$.

Proof. For $m \equiv 0(\bmod 4)$, the result is stated in Lemma 11.20. Let $m=4 n+l$, where $1 \leq l<4$, and $H=\mathrm{GO}_{8 n}^{+}$(3) or $\mathrm{Sp}_{8 n}$ (3). Let $S_{0}$ be a Sylow 2-subgroup of $\mathrm{GO}_{2 l}^{+}(3)$ or $\mathrm{Sp}_{2 l}(3)$. Set $U=H \times S_{0}$. Then $U$ contains a Sylow 2-subgroup of $G$. Let $\chi$ be a 2-vanishing character of $G$. Therefore, $l_{2}(\chi)=l_{2}\left(\left.\chi\right|_{U}\right)$. By Lemma 2.5, if $v$ is a 2-vanishing character of $U$, then $l_{2}(v)=l_{2}(\mu)$ for some 2 -vanishing character $\mu$ of $H$. By Lemma $11.20, l_{2}(\mu) \geq 2$. So $l_{2}(v) \geq 2$, and the result follows. 
Proposition 11.22. Let $G=\mathrm{GO}_{2 m}^{-}(3)$ with $m \geq 5$. Then $G$ has no Steinberg-like character for $p=2$.

Proof. Let $m=4 n+l$, where $1 \leq l \leq 4$, and let $H=\mathrm{GO}_{8 n}^{+}$(3). Then $G$ contains a subgroup $D$ isomorphic to $H \times \mathrm{GO}_{2 l}^{-}(3)$. Then one concludes that $D$ contains a Sylow 2-subgroup of $G$. Let $S_{0}$ be a Sylow 2-subgroup of $\mathrm{GO}_{2 l}^{-}(3)$. Set $U=H \times S_{0}$. Then $U$ contains a Sylow 2-subgroup of $G$. By Lemma 2.5, if $v$ is a 2-vanishing character of $U$, then $l_{2}(v)=l_{2}(\mu)$ for some 2-vanishing character $\mu$ of $H$. So $l_{2}(v) \geq 2$ by Lemma 11.20 , and the result follows.

Proposition 11.23. Let $G=\mathrm{GO}_{2 m+1}(3), m \geq 3$. Then $G$ has no Steinberg-like character for $p=2$.

Proof. The case $m=3$ is dealt with in Lemma 11.17. So we assume that $m>3$, that is, $2 m+1 \geq 9$. Let $m=4 n+l$, where $0 \leq l \leq 3$, and let $H=\mathrm{GO}_{8 n}^{+}$(3). Then $G$ contains a subgroup $D$ isomorphic to $H \times \mathrm{GO}_{2 l+1}$ (3). Then $D$ contains a Sylow 2-subgroup of $G$. Set $U=H \times S_{0}$, where $S_{0}$ is a Sylow 2-subgroup of $\mathrm{GO}_{2 l+1}$ (3), so $U$ contains a Sylow 2-subgroups of $G$. By Lemma 2.5, if $v$ is a 2vanishing character of $U$, then $l_{2}(v)=l_{2}(\mu)$ for some 2-vanishing character $\mu$ of $H$. So $l_{2}(v) \geq 2$ by Lemma 11.20 , and the result follows.

Theorem 11.24. Let $G=\mathrm{GO}_{2 m+1}$ (3) with $m \geq 3, \mathrm{GO}_{2 m}^{ \pm}$(3) with $m \geq 4$, or $\mathrm{Sp}_{2 m}$ (3) with $m \geq 3$, and let $G^{\prime}$ be the derived group of $G$. Let $H$ be a group such that $G^{\prime} \leq H \leq G$. Then $H$ and $H / Z(H)$ have no Steinberg-like character for $p=2$.

Proof. For $H$, this follows from Lemma 11.17, Propositions 11.21, 11.22 and 11.23 using Lemma 2.12, and for $H / Z(H)$, from Lemma 2.5.

We now collect our results to prove our main theorems from the introduction.

Proof of Theorem 1.1. Assume that $G$ is a finite non-abelian simple group possessing a Steinberg-like character $\chi$ with respect to a prime $p$. The cases when $\chi$ is irreducible have been recalled in Proposition 3.1. If Sylow $p$-subgroups of $G$ are cyclic, then $(G, p, \chi)$ is as in Proposition 4.4. So we may now assume that Sylow $p$-subgroups of $G$ are non-cyclic. For $G$ alternating and $p$ odd, there are no cases by Theorem 6.4 except for $A_{6} \cong \mathrm{PSL}_{2}(9)$ with $p=3$. The Steinberg-like characters of sporadic groups are listed in Theorem 5.1.

Thus, $G$ is of Lie type. The case when $p$ is the defining prime was handled in [17] and Propositions 8.1 and 8.2, respectively. So now assume $p$ is not the defining prime for $G$. Groups of exceptional Lie type were handled in Theorem 7.1. For classical groups of large rank with $p$ odd, our result is contained 
in Proposition 9.6, the cases for $\operatorname{PSL}_{n}(q)$ and $\operatorname{PSU}_{n}(q)$ with $p>2$ are completed in Theorem 10.15, and those for the other classical groups in Propositions 10.18 and 10.19. Finally, the cases with $p=2$ are covered by Proposition 11.1 for $G=\operatorname{PSL}_{2}(q)$, Proposition 11.3 for $\operatorname{PSL}_{n}(q)$ and $\operatorname{PSU}_{n}(q)$ with $q \neq 3$, Theorem 11.12 for $\operatorname{PSL}_{n}(3)$ and $\operatorname{PSU}_{n}(3)$, Propositions 11.14, 11.15 and 11.16 for classical groups with $q \neq 3$, and Theorem 11.24 for the case that $q=3$.

Proof of Theorem 1.2. The characters of projective $\overline{\mathbb{F}}_{p} G$-modules of dimension $|G|_{p}$ are in particular Steinberg-like, so, in order to prove this result, we need to go through the list given in Theorem 1.1 (2)-(5). When Sylow $p$-subgroups of $G$ are cyclic, the possibilities are given in Lemma 4.5 (b). For $G$ of Lie type in characteristic $p$, see [23, Theorem 1.1]. Theorem 1.1 (4) is subsumed in statement (1), and finally the alternating groups for $p=2$ are discussed in Theorem 6.14.

\section{Bibliography}

[1] J. Conway, R. Curtis, S. Norton, R. Parker and R. Wilson, Atlas of Finite Groups, Clarendon Press, Oxford, 1985.

[2] C. W. Curtis and I. Reiner, Methods of Representation Theory with Applications to Finite Groups and Orders, Wiley, New York, 1990.

[3] L. Emmett and A. E. Zalesski, On regular orbits of elements of classical groups in their permutation representations, Comm. Algebra 39 (2011), 3356-3409.

[4] W. Feit, The Representation Theory of Finite Groups, North-Holland, Amsterdam, 1982.

[5] E. Giannelli and S. Law, On permutation characters and Sylow $p$-subgroups of $\mathfrak{\subseteq}_{n}$, J. Algebra 506 (2018), 409-428.

[6] L. Héthelyi, M. Shoke and A. Zalesski, On $p$-stability in groups and fusion systems, J. Algebra 492 (2017), 253-297.

[7] R. Howe, On the character of Weil's representation, Trans. Amer. Math. Soc. 177 (1973), 287-298.

[8] B. Huppert, Singer-Zyklen in klassischen Gruppen, Math. Z. 117 (1970), 141-150.

[9] G. James, The Representation Theory of the Symmetric Groups, Springer, Berlin, 1978.

[10] C. Lassueur and G. Malle, Simple endotrivial modules for linear, unitary and exceptional groups, Math. Z. 280 (2015), 1047-1074.

[11] C. Lassueur, G. Malle and E. Schulte, Simple endotrivial modules for quasi-simple groups, J. reine angew. Math. 712 (2016), 141-174. 
[12] F. Lübeck, Character degrees and their multiplicities for some groups of Lie type of rank $<9$, preprint, http://www.math.rwth-aachen.de/ Frank.Luebeck/ chev/DegMult/.

[13] G. Malle and T. Weigel, Finite groups with minimal 1-PIM, Manuscripta Math. 126 (2008), 315-332.

[14] G. Malle and A.E. Zalesski, Prime power degree representations of quasi-simple groups, Arch. Math. 77 (2001), 461-468.

[15] G. Navarro, Characters and Blocks of Finite Groups, Cambridge University, Cambridge, 1998.

[16] H. N. Nguyen, Low dimensional complex characters of the symplectic and orthogonal groups, Comm. Algebra 38 (2010), 1157-1197.

[17] M. Pellegrini and A. E. Zalesski, On characters of Chevalley groups vanishing at the non-semisimple elements, Internat. J. Algebra Comput. 26 (2016), 789-841.

[18] C. Rudloff and A.E. Zalesski, On multiplicity 1 eigenvalues of elements in irreducible representations of finite quasi-simple groups, J. Group Theory 10 (2007), 585-612.

[19] M. Stather, Constructive Sylow theorems for the classical groups, J. Algebra 316 (2007), 536-559.

[20] P. H. Tiep and A.E. Zalesskii, Minimal characters of the finite classical groups, Comm. Algebra 24 (1996), 2093-2167.

[21] A. J. Weir, Sylow $p$-subgroups of the classical groups over finite fields with characteristic coprime to p, Proc. Amer. Math. Soc. 6 (1955), 529-533.

[22] A. E. Zalesski, Minimal polynomials and eigenvalues of $p$-elements in representations of quasi-simple groups with a cyclic Sylow $p$-subgroup, J. Lond. Math. Soc. (2) 59 (1999), 845-866.

[23] A. E. Zalesski, Low dimensional projective indecomposable modules for Chevalley groups in defining characteristic, J. Algebra 377 (2013), 125-156.

[24] A. E. Zalesski, Remarks on Gelfand-Graev characters for simple groups, Arch. Math. 100 (2013), 221-230.

Received February 6, 2019; revised June 14, 2019.

\section{Author information}

Gunter Malle, FB Mathematik, TU Kaiserslautern,

Postfach 3049, 67653 Kaiserslautern, Germany.

E-mail: malle@mathematik .uni-kl.de

Alexandre Zalesski, Department of Physics, Informatics and Mathematics,

National Academy of Sciences of Belarus, Minsk, Belarus.

E-mail: alexandre.zalesski@gmail.com 